\title{
QUASI-ORDINARY POWER SERIES AND THEIR ZETA FUNCTIONS
}

\author{
E. ARTAL BARTOLO, PI. CASSOU-NOGUÈ, I. LUENGO, AND A. MELLE HERNÁNDEZ
}

\begin{abstract}
The main objective of this paper is to prove the monodromy conjecture for the local Igusa zeta function of a quasi-ordinary polynomial of arbitrary dimension defined over a number field. In order to do it, we compute the local Denef-Loeser motivic zeta function $Z_{\mathrm{DL}}(h, T)$ of a quasi-ordinary power series $h$ of arbitrary dimension over an algebraically closed field of characteristic zero from its characteristic exponents without using embedded resolution of singularities. This allows us to effectively represent $Z_{\mathrm{DL}}(h, T)=P(T) / Q(T)$ such that almost all the candidate poles given by $Q(T)$ are poles. Anyway, these candidate poles give eigenvalues of the monodromy action of the complex of nearby cycles on $h^{-1}(0)$. In particular we prove in this case the monodromy conjecture made by Denef-Loeser for the local motivic zeta function and the local topological zeta function. As a consequence, if $h$ is a quasi-ordinary polynomial defined over a number field we prove the Igusa monodromy conjecture for its local Igusa zeta function.
\end{abstract}

\section{INTRODUCTION}

Let $h$ be a polynomial in $\mathbb{Z}\left[x_{1}, \ldots, x_{d}\right]$ and fix $p \in \mathbb{Z}$ a prime number. In order to study the number $N_{k}$ of solutions of the congruence $h \equiv 0 \bmod p^{k}$, classically one associates with $h$ the Poincaré series

$$
P(T)=\sum_{k=0}^{\infty} N_{k} T^{k} .
$$

J. Igusa proved in 22] that $P(T)$ is a rational function on $T$ by relating it with the following $p$-adic integral

$$
I(h, s):=\int_{\mathbb{Z}_{p}^{d}}|h(x)|^{s}|d x|,
$$

for $s \in \mathbb{C}, \operatorname{Re}(s)>0$, where $|d x|$ denotes the Haar measure on $\mathbb{Q}_{p}^{d}$ normalized in such of way that $\mathbb{Z}_{p}^{d}$ is of volume 1 .

Igusa proved the identity $P\left(p^{-d-s}\right)=\frac{1-p^{-s} I(h, s)}{1-p^{-s}}$ and he used an embedded resolution of $h^{-1}(0)$ to show that $I(h, s)$ is a rational function on $p^{-s}$, (see [7] for a proof

2000 Mathematics Subject Classification. 14B05,14E15,32S50.

Key words and phrases. Motivic, topological and Igusa zeta functions, monodromy, quasiordinary singularities.

First author is partially supported by BFM2001-1488-C02-02; the last two authors are partially supported by BFM2001-1488-C02-01. 
without resolution of singularities). In fact each exceptional divisor of an embedded resolution gives a candidate pole of $I(h, s)$ but many of them are not actually poles.

Igusa conjectured that poles of $I(h, s)$ are related with eigenvalues of the complex monodromy at some point of $h^{-1}(0)$, see [8]. More precisely he raised the conjecture:

Igusa Monodromy Conjecture. If $h \in F\left[x_{1}, \ldots, x_{d}\right] \backslash F$, for some number field $F \subset \mathbb{C}$, then for almost all p-adic completion $K$ of $F$, if $s_{0}$ is a pole of $I(h, K, s)$, then $\exp \left(2 i \pi \Re\left(s_{0}\right)\right)$ is an eigenvalue of the local monodromy of $h$ at some complex point of $h^{-1}(0)$.

Since then, some partial results have been obtained, see the Bourbaki Seminar talk by J. Denef [8] for a survey of these results until 1991, ([25, 26, 29, 2]). We recall two cases where Igusa monodromy conjecture has been proved. In fact in both cases, the following stronger version of the Igusa monodromy conjecture was proved: for almost all $p$-adic completion $K$ of $F$, if $s_{0}$ is a pole of $I(h, K, s)$, then $\Re\left(s_{0}\right)$ is a root of the Bernstein polynomial $b_{h}(s)$ of $h$. Firstly, F. Loeser in [25] gave a proof for reduced polynomials in two variables. One other interesting case is the case of polynomials (in arbitrary dimension) non-degenerated with respect to their Newton polyhedron and verifying some "resonance" conditions. This result was also proved by F. Loeser in [26]. For plane curves, the strong candidate poles come from the rupture components in an embedded resolution of $h^{-1}(0)$, see 33 . For nondegenerated polynomials J. Denef gave a set of strong candidate poles which comes from faces of codimension 1 in the Newton polyhedron of $h$, see [9].

In this paper we will prove the Igusa monodromy conjecture for the local Igusa zeta function $I_{0}(h, K, s)$, which is the local version of $I(h, K, s)$, for quasi-ordinary polynomials in arbitrary dimension. As we will see quasi-ordinary polynomials behave in many aspects as plane curves. In our proof we use in a essential way motivic integration on the space of arcs on an algebraic variety.

Motivic measure take values in a completion of the Grothendieck ring of algebraic varieties. Let $K_{0}\left(\operatorname{Var}_{k}\right)$ be the Grothendieck ring of algebraic varieties over $k$. Let $\mathbb{L}=\left[\mathbb{A}_{k}^{1}\right]$ denote the class in $K_{0}\left(\operatorname{Var}_{k}\right)$ of the affine line. The naive motivic ring $\mathcal{M}_{k}$ of algebraic varieties over $k$ is the polynomial ring $\mathcal{M}_{k}=K_{0}\left(\operatorname{Var}_{k}\right)\left[\mathbb{L}^{-1}\right]$.

Let $X$ be a non singular irreducible complex algebraic variety of pure dimension $d$. For any $n \in \mathbb{N}$, let $\mathcal{L}_{n}(X)$ denote the space of arcs modulo $t^{n+1}$ on $X$; it has a structure of complex variety. The arc space $\mathcal{L}(X)$ of $X$ is the projective limit of the algebraic varieties $\mathcal{L}_{n}(X)$. For any $n \in \mathbb{N}$, let $\pi_{n}: \mathcal{L}(X) \rightarrow \mathcal{L}_{n}(X)$ be the natural projection. For any arc $\boldsymbol{\varphi} \in \mathcal{L}(X)$, the origin of the arc is $\pi_{0}(\boldsymbol{\varphi})$. For each closed point $x \in X$, let $\mathcal{L}_{x}(X)$ (resp. $\left.\mathcal{L}_{n, x}(X)\right)$ be the set of arcs on $X$ (resp. truncated arcs) with origin at $x$.

Consider $X=\mathbb{A}^{d}$ the $d$-dimensional complex affine space and $x=\mathbf{0}$ its origin. Let $h \in \mathbb{C}\left[x_{1}, \ldots, x_{d}\right]$ be a complex polynomial, with $h(\mathbf{0})=0$. Set $V_{n}:=\{\boldsymbol{\varphi} \in$ $\left.\mathcal{L}_{x}(X): \operatorname{ord}(h \circ \varphi)=n\right\}$. Denef and Loeser in [15] defined the naive motivic zeta function of $h$ by

$$
Z_{\text {naive }}(h, T):=\sum_{n \geq 1}\left[\pi_{n}\left(V_{n}\right)\right] \mathbb{L}^{-n d} T^{n} \in \mathcal{M}_{k}[[T]]
$$


In fact we will consider the local Denef-Loeser motivic zeta function which is nothing but $Z_{D L}(h, T)=\mathbb{L}^{-d} Z_{\text {naive }}(h, T)$. They showed in 13, using embedded resolution of singularities, that $Z_{D L}(h, T)$ is a rational function. It belongs to the subring $\mathcal{N}$ of the ring $\widehat{\mathcal{M}}_{k}[[T]]$ which is generated by the image in $\widehat{\mathcal{M}}_{k}[[T]]$ of $\mathcal{M}_{k}[T]$ and $\left(1-\mathbb{L}^{-a} T^{b}\right)^{-1}, a, b \in \mathbb{N}, b>0$. The monodromy conjecture in this case states, see [13, section 2.4]:

Motivic Monodromy Conjecture. There is a set $S=\{(a, b): a, b \in \mathbb{N}, b>0\}$ such that $Z_{D L}(h, T) \in \mathcal{M}_{k}[T]\left[\left(1-\mathbb{L}^{-a} T^{b}\right)^{-1}\right]_{(a, b) \in S}$ and if $q=a / b,(a, b) \in S$, then $\exp (2 i \pi q)$ is an eigenvalue of the local complex algebraic monodromy around zero at some $P \in h^{-1}(0)$.

It turns out that $Z_{D L}(h, T)$ is the right function to study several monodromy conjectures because it specializes to the local Igusa zeta function and to the local topological zeta function:

- If $h$ is a non-constant polynomial defined over a number field $F$ then it follows from [13, see also [15], that for almost all finite places of $F$, the real parts $N s+\nu$ of poles of $I_{0}(h, K, s)$ come from factors $\left(1-\mathbb{L}^{-\nu} T^{N}\right)$ in the denominator of $Z_{\text {naive }}(h, T)$.

- The local topological zeta function $Z_{\text {top }, 0}(h, s)$ of a complex polynomial $h$ was introduced by Denef and Loeser in [12] as a kind of limit of the local Igusa zeta function. Later on, they obtained $Z_{\text {top }, 0}(h, s)$ from $Z_{D L}(h, T)$ by the following procedure, cf. 13, $\S 2.3]$. First substitute $T$ by $\mathbb{L}^{-s}$ in $Z_{D L}(h, T)$, then expand $\mathbb{L}^{-s}$ and $(\mathbb{L}-1)\left(1-\mathbb{L}^{-\nu+N s}\right)^{-1}$ into series in $\mathbb{L}-1$. Finally take the usual Euler characteristic $\chi_{\text {top }}$ (this works because $\chi_{\text {top }}(\mathbb{L})=1$ ). Then the local topological zeta function is the following rational function, see [15, 13]:

$$
Z_{\text {top }, 0}(h, s):=\chi_{\text {top }}\left(Z_{D L}\left(\mathbb{L}^{-s}\right)\right) .
$$

It is clear that poles of $Z_{\text {top }, 0}(h, s)$ induce poles of $Z_{D L}(h, T)$. The other way around is not true, see Example 3.10. In this case Denef and Loeser conjectured in [12]:

Topological Monodromy Conjecture. If $s_{0}=\nu+s N$ is a pole of $Z_{\mathrm{top}, 0}(h, s)$ then $\exp (2 i \pi(-\nu / N))$ is an eigenvalue of the monodromy action at some point of $h^{-1}(0)$.

One result of this paper is that if $h$ defines a quasi-ordinary singularity of hypersurface of arbitrary dimension then the monodromy conjecture for $Z_{D L}(h, T)$ is true, see Corollary 17.5. This fact implies that the Igusa monodromy conjecture for $I_{0}(h, K, s)$ and the Denef-Loeser monodromy conjecture for $Z_{\mathrm{top}, 0}(h, s)$. In fact we prove a stronger result.

A germ of a complex analytic variety $(V, 0)$ is called a quasi-ordinary singularity if there is a finite morphism (proper with finite fiber map) of analytic germs $\pi$ : $(V, 0) \rightarrow\left(\mathbb{C}^{d}, 0\right)$ whose discriminant locus is contained in $x_{1} x_{2} \ldots x_{d}=0$, for some local coordinates $\left(x_{1}, \ldots, x_{d}\right)$. We are only interested in quasi-ordinary hypersurface singularities $(V, 0) \subset\left(\mathbb{C}^{d+1}, 0\right)$.

A convergent power series $h \in \mathbb{C}\{\mathbf{x}, z\}, h(0)=0$, defines a quasi-ordinary singularity at zero if the germ of its zero locus $(V, 0) \subset\left(\mathbb{C}^{d+1}, 0\right)$ is a quasi-ordinary 
singularity. These singularities behave in many aspects as singularities of plane curves mainly because, after Jung-Abhyankar theorem, they admit fractional power series parameterizations and a finite set of characteristic exponents, see [1], [27. J. Lipman and Y. Gau proved that in the irreducible case these exponents determine the embedded topology of $(V, 0)$, [17, 24]. Recently, several embedded resolutions constructed from the characteristic exponents have been obtained, see the works of O. Villamayor [35] or the Ph.D. of P.D. González Pérez [18].

In [27], I. Luengo showed that the usual Newton-Puiseux method for curves can be used in arbitrary dimension to find the roots with fractional exponents of a quasi-ordinary polynomial by means of Newton maps (see paragraph 4.4). The key point to find the roots is that after a Newton map we get a new quasi-ordinary polynomial with less characteristic exponents and one proceeds by finite induction. In this paper we do not use embedded resolution of quasi-ordinary singularities to compute $Z_{D L}(h, T)$ but the above procedure given by Newton maps. Such a method allow us to decompose $Z_{D L}(h, T)$ as a sum of two rational motivic zeta functions:

$$
Z_{D L}(h, T)=Z_{D L}^{A}(h, T)+Z_{D L}^{B}(h, T) .
$$

The $A$-part corresponds to $\operatorname{arcs} \varphi \in \mathcal{L}_{0}(X)$ such that the $t$-order $\operatorname{ord}_{t}(h \circ \varphi)$ can be computed from the (degenerated or not) Newton polytope of $h$. The computation of $Z_{D L}^{A}(h, T)$ from the Newton polytope follows ideas of J. Denef and K. Hoornaert in the $p$-adic case, see [10]. In fact we will show an interesting description of $Z_{D L}^{A}(h, T)$ in terms of generating functions of some rational polyhedra obtained from the Newton polytope of $h$, see sections 2 and 3. As an application a formula for $Z_{D L}(h, T)$ for any germ of complex analytic function $h$ with non-degenerated Newton polytope is given, see theorem 3.4. G. Guibert has recently obtained a similar formula, see [20].

In order to compute the measure of the $\operatorname{arcs} \varphi \in \mathcal{L}_{0}(X)$ in the $B$-part we pull back these arcs under Newton maps. In particular $Z_{D L}^{B}(h, T)$ is the sum of some motivic zeta functions depending on the pull-back of $h$ under all its Newton maps. There is here one major technical problem. In dimension higher that 1, usually there exist some arcs which cannot be lifted under usual Newton maps. To solve this problem we need to consider Newton maps with coefficients in $\mathbb{C}\{t\}$. Thus our results really deal with quasi-ordinary power series with coefficients in $\mathbb{C}\{t\}$ instead of $\mathbb{C}$. Now Newton maps are $\mathbb{C}\{t\}$-morphisms in the terminology introduced by Denef and Loeser in [16] and we can apply the change of variables formula. The differential form plays a role here but throughout this introduction we omit it. In fact, we perform all these computations for an algebraically closed field $k$ of characteristic zero.

Essentially in $Z_{D L}^{B}(h, T)$ we get quasi-ordinary singularities with less characteristic exponents and we can apply recursively this formula. In this way, for $Z_{\text {top }, 0}(h, s)$ a very effective and closed recursive formula is given only depending on the tree of characteristic exponents, cf. Theorem 6.3. For $Z_{D L}(h, T)$ the formula, also recursive, is enough to give a short list of candidate poles. Each characteristic exponent is a rational $d$-tuple. Each non-zero coordinate of each characteristic exponent will give a candidate pole of $Z_{D L}(h, T)$. In section 6 some of them are excluded to get a 
smallest set $S C P(h)$ of strong candidate poles. Our main result is

$$
Z_{D L}(h, T) \in \mathbb{Z}\left[\mathbb{L}, \mathbb{L}^{-1},\left(1-\mathbb{L}^{-\nu} T^{N}\right)^{-1}\right][T]_{(N, \nu) \in S C P(h)} .
$$

The proof of the above result gives some extra information. Namely all elements in $S C P(h)$, but one concrete case $(*)$ when $d=2$ (cf. Proposition 6.10), appear also as strong candidate poles of a transversal section of $h$ at some point of the singular locus of $h^{-1}(0)$. Transversal sections at generic points are also quasi-ordinary singularities, this fact allows us to prove the motivic monodromy conjecture by induction on the dimension. For curves, this method gives a closed formula for $Z_{D L}(h, T)$, cf. (6.1), and from this formula a new proof of the monodromy conjecture follows directly. In the $(*)$-case, there is only one strong candidate pole not appearing in the transversal sections. We use the formula, proved by P.D. González Pérez, L.J. McEwan and A. Némethi in [19], for the zeta function of the monodromy at the origin of quasiordinary singularities to show that this strong candidate pole also gives an eigenvalue of the monodromy of $h$ at the origin.

The computation of $Z_{\text {top }, 0}(h, s)$ for quasi-ordinary singularities gives a very effective way to compute the poles of $Z_{\text {top }, 0}(h, s)$ for a general surface singularity. To short this work we will provide the details in a forthcoming paper. The basic idea is to use the well-known Jung-method as follows.

Let $p:(S, 0) \subset\left(\mathbb{C}^{3}, 0\right) \rightarrow\left(\mathbb{C}^{2}, 0\right)$ be a finite morphism of a (hyper)surface singularity $(S, 0)$, defined by $h$, with discriminant locus $(\Delta, 0) \subset\left(\mathbb{C}^{2}, 0\right)$. Take an embedded resolution $\pi:\left(\widetilde{\mathbb{C}^{2}}, \mathcal{D}\right) \rightarrow\left(\mathbb{C}^{2}, 0\right)$ of the germ of curve $(\Delta, 0)$ where the exceptional locus $\mathcal{D}=\pi^{-1}(0)$ is a normal crossing divisor. The pull-back $\tilde{p}:(\tilde{S}, \mathcal{E}) \rightarrow\left(\widetilde{\mathbb{C}^{2}}, \mathcal{D}\right)$ is a finite map whose discriminant is contained in the pull-back of $(\Delta, 0)$. Thus the singularities of $(\tilde{S}, \mathcal{E})$ are all quasi-ordinary. We have proved that the poles of $Z_{\text {top }, 0}(h, s)$ are contained in the set of poles of either the transversal sections (now curves) at the rupture components of the resolution or components of the singular locus of $(S, 0)$. This gives a short list of strong candidate poles which are in general poles of $Z_{\mathrm{top}, 0}(h, s)$. Next step will be to prove that they induce eigenvalues of the monodromy.

\section{Contents}

\begin{tabular}{|lr|}
\hline 1. Introduction & 1 \\
\hline 2. Motivic integration & 6 \\
\hline 2.1. Grothendieck ring of varieties & 6 \\
\hline 2.2. The arc space of a varietv & 7 \\
\hline 2.3. Local Denef-Loeser motivic zeta function & 8 \\
\hline 3. Generating functions and Newton polvhedron & 9 \\
\hline 3.1. Generating functions for integer points in rational polvhedra & 9 \\
\hline 3.2. Motivic zeta function and Newton polvhedra & 11 \\
\hline 4. Quasi-ordinary power series & 18 \\
\hline 4.1. Characteristic exponents & 19
\end{tabular}


4.2. Newton polvhedron and good coordinates 20

4.3. Dual decomposition 23

4.4. Newton map associated with a Newton component 26

4.5. Transversal sections of a quasi-ordinary power series 31

5. Denef-Loeser motivic zeta function under the Newton maps 34

5.1. Vertices of the dual decomposition 40

5.2. Edges of the Newton polvtope 43

5.3. Zeta functions along strata 53

6. Consequences of the main theorems 54

6.1. Essential variables 54

6.2. Curves case $\quad 55$

6.3. The topological zeta function 55

$\begin{array}{ll}\text { 6.4. A special candidate pole } & 57\end{array}$

7. Monodromv conjecture for quasi-ordinary power series 68

$\begin{array}{lll}\text { 7.1. Monodromv conjecture for curves } & 68\end{array}$

7.2. Monodromv coniecture: general case 71

7.3. Monodromy conjecture for the Igusa zeta-function 72

$\begin{array}{ll}\text { References } & 73\end{array}$

Conventions. Throughout this paper we denote by $\mathbb{N}$ the set of the nonnegative integers, $\mathbb{P}$ the set of positive integers, $\mathbb{R}_{+}=\{x \in \mathbb{R}: x \geq 0\}$ and $\mathbb{R}_{>0}=\{x \in \mathbb{R}$ : $x>0\}$. To shorten the notation we will use bold symbols for $d$-tuples, for instance $\mathbf{x}=\left(x_{1}, \ldots, x_{d}\right)$.

In this paper we work over a field $k$ of characteristic zero. A variety over $k$ will mean a reduced separated scheme of finite type over the field $k, \mathbf{G}_{m, k}^{d}:=$ Spec $k\left[x_{1}, \ldots, x_{d}, x_{1}^{-1}, \ldots, x_{d}^{-1}\right]$ denote the $d$-dimensional torus over $k$ and $\mathbb{A}_{k}^{d}:=$ Spec $k\left[x_{1}, \ldots, x_{d}\right]$ the $d$-dimensional affine space over $k$.

\section{Motivic integration}

In this section we recall several results form [13, 14, 15, 16. We refer to these papers for the proofs of such results. In the first two section we work over a field $k$ of characteristic zero.

\subsection{Grothendieck ring of varieties.}

The Grothendieck ring of algebraic varieties over $k$, denoted by $K_{0}\left(\operatorname{Var}_{k}\right)$, is the free Abelian group on isomorphism classes $[X]$ of algebraic varieties $X$ over $k$ subject to the relations $[X]=[X-Y]+[Y]$ where $Y \subset X$ is a closed subvariety of $X$. The Cartesian product of varieties gives the ring structure. The following properties that we will freely use throughout the paper, hold in $K_{0}\left(\operatorname{Var}_{k}\right)$.

(1) If $f: Y \rightarrow Z$ is a fibre bundle with fibre $F$ which is locally trivial in the Zariski topology, then $[Y]=[F][Z]$. 
(2) If a variety $X$ is partitioned by locally closed subvarieties $X_{1}, \ldots, X_{n}$, then $[X]=\left[X_{1}\right]+\ldots+\left[X_{n}\right]$.

(3) If $f: Y \rightarrow Z$ is a bijective morphism, then $[Y]=[Z]$. The proof of this property is deduced from the proof of the same property at the level of virtual Hodge polynomials which can be found for instance in 6 .

Let $\mathbb{L}=\left[\mathbb{A}_{k}^{1}\right]$ denote the class in $K_{0}\left(\operatorname{Var}_{k}\right)$ of the affine line. The naive motivic ring $\mathcal{M}_{k}$ of algebraic varieties over $k$ is the polynomial $\operatorname{ring} \mathcal{M}_{k}=K_{0}\left(\operatorname{Var}_{k}\right)\left[\mathbb{L}^{-1}\right]$. Let $F^{m} \mathcal{M}_{k}$ denote the subgroup of $\mathcal{M}_{k}$ generated by $[X] \mathbb{L}^{-i}$ with $\operatorname{dim} X-i \leq-m$ and $\widehat{\mathcal{M}}_{k}$ denote the completion of $\mathcal{M}_{k}$ with respect to the filtration $F$. This completion was first introduced by M. Kontsevich.

\subsection{The arc space of a variety.}

Let $X$ be a nonsingular irreducible algebraic variety over $k$ of pure dimension $d$. For any $n \in \mathbb{N}$, let $\mathcal{L}_{n}(X)$ denote the space of arcs modulo $t^{n+1}$ on $X$ which has a structure of $k$-variety, whose $K$-rational points, for any field $K$ containing $k$, are the $K[t] /\left(t^{n+1}\right) K[t]$-rational points of $X$. The arc space $\mathcal{L}(X)$ of $X$ is the projective limit of the algebraic varieties $\mathcal{L}_{n}(X)$. For any $n \in \mathbb{N}$, let $\pi_{n}: \mathcal{L}(X) \rightarrow \mathcal{L}_{n}(X)$ be the natural projection. For any $\operatorname{arc} \varphi \in \mathcal{L}(X)$, the origin of the arc is $\pi_{0}(\varphi)$. For any closed point $x \in X$, let $\mathcal{L}_{x}(X)$ (resp. $\left.\mathcal{L}_{n, x}(X)\right)$ be the arcs (resp. truncated arcs) with origin at $x$. The above definitions extend to the case where $X$ is a reduced and separated scheme of finite type over $k[t]$. For any $n \in \mathbb{N}, \mathcal{L}_{n}(X)$ is the $k$-scheme which represents the functor

$$
R \mapsto \operatorname{Mor}_{k[t]-\text { schemes }}\left(\operatorname{Spec} R[t] / t^{n+1} R[t], X\right)
$$

defined in the category of $k$-algebras and again $\mathcal{L}(X)$ is its projective limit. The truncation map will be also denoted by $\pi_{n}: \mathcal{L}(X) \rightarrow \mathcal{L}_{n}(X)$.

Let $A$ be a semialgebraic, resp. $k[t]$-semialgebraic, subset of $\mathcal{L}(X)$; it is called stable at level $n \in \mathbb{N}$ if $A=\pi_{n}^{-1} \pi_{n}(A)$. We remark that if $A$ is stable at level $n$ then it is stable at level $n^{\prime} \geq n$. The set $A$ is called stable if it is stable at some level $n$. A subset $A \in \mathcal{L}(X)$ is cylindrical at level $n$ if $A=\pi_{n}^{-1}(C)$ with $C$ a constructible set, and $A$ is cylindrical if it is cylindrical at some level. Denote by $\mathbf{B}^{t}$ the set of all $k[t]$-semialgebraic subsets of $\mathcal{L}(X)$.

The motivic measure on $\mathcal{L}(X)$ is the unique map $\mu_{X}: \mathbf{B}^{t} \rightarrow \widehat{\mathcal{M}}_{k}$ such that:

(a) If $A \in \mathbf{B}^{t}$ is stable at level $n$, then $\mu_{X}(A)=\left[\pi_{n}(A)\right] \mathbb{L}^{-(n+1) d}$.

(b) If $A \in \mathbf{B}^{t}$ is contained in $\mathcal{L}(S)$ with $S$ a reduced closed subscheme of $X \otimes_{k} k[t]$ with $\operatorname{dim}_{k[t]} S<\operatorname{dim} X$, then $\mu_{X}(A)=0$.

(c) Let $A_{i} \in \mathbf{B}^{t}$ for all $i \in \mathbb{N}$. Assume that the $A_{i}$ 's are mutually disjoint and that $A:=\cup_{i \in \mathbb{N}} A_{i}$ is $k[t]$-semialgebraic. Then $\sum_{i \in \mathbb{N}} \mu_{X}\left(A_{i}\right)$ converges in $\widehat{\mathcal{M}}_{k}$ to $\mu_{X}(A)$.

Because there might exist cylindrical subsets of $\mathcal{L}(X)$ which are not semialgebraic the motivic measure has been extended to a measure, also denoted $\mu_{X}$, defined over the Boolean algebra of the measurable subsets of $\mathcal{L}(X)$, see [16, Appendix]. The above properties hold for measurable subsets of $\mathcal{L}(X)$ too. 
For a measurable subset $A$ in $\mathcal{L}(X)$ and a function $\alpha: A \rightarrow \mathbb{Z} \cup\{\infty\}$, we say that $\mathbb{L}^{-\alpha}$ is integrable on $A$ if the fibres of $\alpha$ are measurable, $\alpha^{-1}(\infty)$ has measure zero and the motivic integral

$$
\int_{A} \mathbb{L}^{-\alpha} d \mu_{X}:=\sum_{n \in \mathbb{Z}} \mu_{X}\left(A \cap \alpha^{-1}(n)\right) \mathbb{L}^{-n} \in \widehat{\mathcal{M}}_{k}
$$

converges in $\widehat{\mathcal{M}}_{k}$.

Definition 2.1. Let $X$ and $Y$ be $k$-varieties. A function $\pi: \mathcal{L}(Y) \rightarrow \mathcal{L}(X)$ will be call a $k[t]$-morphism if it is induced by a morphism of $k[t]$-schemes $Y \otimes_{k} k[t] \rightarrow$ $X \otimes_{k} k[t]$.

Theorem 2.2 (Change variables formula). Let $X$ and $Y$ be smooth $k$-varieties of pure dimension d. Let $\pi: \mathcal{L}(Y) \rightarrow \mathcal{L}(X)$ be a $k[t]$-morphism. Let $A$ and $B$ be $k[t]$-semialgebraic subsets of $\mathcal{L}(X)$ and $\mathcal{L}(Y)$, respectively. Assume that $\pi$ induces a bijection between $B$ and $A$. Then, for any function $\alpha: A \rightarrow \mathbb{Z} \cup\{\infty\}$ such that $\mathbb{L}^{-\alpha}$ is integrable on $A$, we have

$$
\int_{A} \mathbb{L}^{-\alpha} d \mu_{X}=\int_{B} \mathbb{L}^{-\alpha \circ \pi-\operatorname{ord}_{t} J_{\pi}(y)} d \mu_{Y}
$$

where $\operatorname{ord}_{t} J_{\pi}(y)$, for any $y \in \mathcal{L}(Y)$, denotes the $t$-order of the Jacobian of $\pi$ at $y$.

\subsection{Local Denef-Loeser motivic zeta function.}

Let $h \in k\left[\left[x_{1}, \ldots, x_{d}\right]\right]$ be a formal power series in the maximal ideal of the formal power series ring. Let $X:=\mathbb{A}_{k}^{d}$ be the $d$-dimensional affine space and $x=\mathbf{0}$ its origin. Set $V_{n}:=\left\{\boldsymbol{\varphi} \in \mathcal{L}_{x}(X): \operatorname{ord}(h \circ \boldsymbol{\varphi})=n\right\}$. The local Denef-Loeser motivic zeta function of $h$ is the power series

$$
Z_{D L}(h, T):=\sum_{n \geq 1} \mu_{X}\left(V_{n}\right) T^{n} \in \mathcal{M}_{k}[[T]] .
$$

Since $V_{n}$ is a stable semialgebraic set at level $n$ of $\mathcal{L}_{n, x}(X)$, then we have $\mu_{X}\left(V_{n}\right)=$ $\left[\pi_{n}\left(V_{n}\right)\right] \mathbb{L}^{-(n+1) d} \in \mathcal{M}_{k}$. In fact $Z_{D L}(h, T)$ belongs to the subring $\mathcal{N}$ of the ring $\widehat{\mathcal{M}}_{k}[[T]]$ which is generated by the image in $\widehat{\mathcal{M}}_{k}[[T]]$ of $\mathcal{M}_{k}[T]$ and $\left(1-\mathbb{L}^{-a} T^{b}\right)^{-1}, a \in$ $\mathbb{N}, b \in \mathbb{P}$. Denef and Loeser in [15] introduced the naive motivic zeta function of $h$ as

$$
Z_{\text {naive }}(h, T):=\sum_{n \geq 1}\left[\pi_{n}\left(V_{n}\right)\right] \mathbb{L}^{-n d} T^{n},
$$

then $\mathbb{L}^{d} Z_{D L}(h, T)=Z_{\text {naive }}(h, T)$.

We will work in a slightly more general set up. In order to be able to use the change variables formula we consider $Z_{D L}(h, \omega, T)$ where $\omega$ is a regular differential form on $X$ and the pair $(h, \omega)$ will verify the following condition.

Support Condition 2.3. The pair $(h, w)$ satisfies the support condition if and only if

(1) $h(\mathbf{x})=\prod_{j=1}^{d} x_{j}^{N_{j}} f(\mathbf{x}), N_{j} \in \mathbb{N}$, with $x_{j}$ does not divide $f$ for any $j=1, \ldots, d$, 
(2) $\omega$ is a regular differential form of type $\omega=\prod_{j=1}^{d} x_{j}^{\nu_{j}-1} d x_{1} \wedge \ldots \wedge d x_{d}, \nu_{j} \geq 1$, (3) $N_{j}=0$ implies $\nu_{j}=1$, for any $j=1, \ldots, d$.

Set $V_{n, m}:=\left\{\boldsymbol{\varphi} \in V_{n} \mid \operatorname{ord}(\omega \circ \boldsymbol{\varphi})=m\right\}$. For a given $n$, there are finitely many $m$ such that $V_{n, m} \neq \emptyset$, because of the support condition, cf. 34. The local DenefLoeser motivic zeta function of a pair $(h, \omega)$ is the rational function

$$
Z_{D L}(h, w, T):=\sum_{n \in \mathbb{N}}\left(\sum_{m \in \mathbb{N}} \mathbb{L}^{-m} \mu_{X}\left(V_{n, m}\right)\right) T^{n} \in \mathcal{N} .
$$

The local topological zeta function $Z_{\text {top }, 0}(h, \omega, s)$ is obtained from $Z_{D L}(h, w, T)$ by the following procedure, see [13, $\S 2.3]$. First substitute $T$ by $\mathbb{L}^{-s}$ in $Z_{D L}(h, \omega, T)$, then expand $\mathbb{L}^{-s}$ and $(\mathbb{L}-1)\left(1-\mathbb{L}^{-\nu+N s}\right)^{-1}$ into series in $\mathbb{L}-1$. Finally take the usual Euler characteristic $\chi_{\text {top }}$ in étale $\mathbf{Q}_{\ell^{-}}$cohomology, this works because $\chi_{\text {top }}(\mathbb{L})=1$. Then $Z_{\text {top }, 0}(h, \omega, s)$ is the rational function

$$
Z_{\text {top }, 0}(h, \omega, s):=\chi_{\text {top }}\left(Z_{D L}\left(h, \omega, \mathbb{L}^{-s}\right)\right) .
$$

We will use the symbol $\chi_{\text {top }}\left(\bullet\left(\mathbb{L}^{-s}\right)\right)$ to denote the composition of the above three operations whenever it has sense.

Remark 2.4. See [16, Remark 1.19] to generalize the results presented here to schemes over $k[[t]]$ instead over $k[t]$.

\section{Generating functions And NeWton polyhedron}

\subsection{Generating functions for integer points in rational polyhedra.}

In this section some well known facts about generating functions of rational polyhedra are reviewed. We use as a reference [31, Section 4.6] and [3].

Let $\mathbb{R}^{d}$ be the Euclidean $d$-space with the standard scalar product $\mathbf{x} \cdot \mathbf{y}=\sum_{l=1}^{d} x_{l} y_{l}$. A rational polyhedron $P \subset \mathbb{R}^{d}$ is the set of solutions of a finite system of linear inequalities with integer coefficients:

$$
P:=\left\{\mathbf{x} \in \mathbb{R}^{d}: \boldsymbol{\beta}_{i} \cdot \mathbf{x} \leq c_{i} \text { for } i=1, \ldots, m\right\}, \text { where } \boldsymbol{\beta}_{i} \in \mathbb{Z}^{d} \text { and } c_{i} \in \mathbb{Z} .
$$

A bounded rational polyhedron is called a polytope. A nonempty polyhedron is called a cone if $\lambda \mathbf{x} \in P$ whenever $x \in P$ and $\lambda \geq 0$. A pointed polyhedral cone is a cone which does not contain a line.

The algebra of polyhedra $\mathcal{P}\left(\mathbb{R}^{d}\right)$ is the $\mathbb{Q}$-vector space spanned by the indicator functions $[P]$ of all polyhedra $P \subset \mathbb{R}^{d}$, where the indicator function $[P]: \mathbb{R}^{d} \rightarrow \mathbb{R}$ of $P$ is defined by

$$
[P](x)= \begin{cases}1 & \text { if } x \in P \\ 0 & \text { if } x \notin P .\end{cases}
$$

We will use the same notation $[\bullet]$ for indicator functions and for elements in the Grothendieck ring of algebraic varieties, nevertheless we hope no confusion will arise. 
Let $P \subset \mathbb{Q}^{d}$ be a rational polyhedron, with the set of integral points in $P$ we associate the generating function

$$
\Phi_{P}(\mathbf{x}):=\sum_{\boldsymbol{\alpha} \in P \cap \mathbb{Z}^{d}} x_{1}^{\alpha_{1}} \ldots x_{d}^{\alpha_{d}} .
$$

These series define a map $\Phi: \mathcal{P}\left(\mathbb{Q}^{d}\right) \rightarrow \mathbb{Q}(\mathbf{x})$ with the following properties:

(1) if $P_{1}, \ldots, P_{r} \subset \mathbb{R}^{d}$ are rational polyhedra whose indicator functions satisfy a linear identity $\alpha_{1}\left[P_{1}\right]+\ldots+\alpha_{r}\left[P_{r}\right]=0$, with $\alpha_{i} \in \mathbb{Q}$, then

$$
\alpha_{1} \Phi_{P_{1}}(\mathbf{x})+\ldots+\alpha_{r} \Phi_{P_{r}}(\mathbf{x})=0 .
$$

(2) If $\mathbf{g}+P$ is a translation of $P$ by an integer vector $\mathbf{g} \in \mathbb{Z}^{d}$ then $\Phi_{\mathbf{g}+P}(\mathbf{x})=$ $\mathrm{x}^{\mathrm{g}} \Phi_{P}(\mathrm{x})$.

(3) $\Phi_{\{\mathbf{0}\}}(\mathbf{x})=1$.

Let $\mathcal{C}$ be a pointed polyhedral cone. The one dimensional faces of $\mathcal{C}$ are called extreme rays. A pointed polyhedral cone has only finitely many extreme rays and it is the convex hull of its extreme rays. A simplicial cone $\sigma$ is an e-dimensional pointed convex polyhedral cone with $e$ extreme rays; it may be also defined as a cone generated by $e$ linearly independent integer vectors $\boldsymbol{\beta}_{1}, \ldots, \boldsymbol{\beta}_{e}$, thus $\sigma=$ $\left\{\lambda_{1} \boldsymbol{\beta}_{1}+\ldots+\lambda_{e} \boldsymbol{\beta}_{e}, \lambda_{i} \in \mathbb{R}_{+}\right\}$.

A triangulation of $\mathcal{C}$ consists of a finite collection $\Gamma=\left\{\sigma_{1}, \ldots, \sigma_{t}\right\}$ of simplicial cones such that

i) $\cup \sigma_{i}=\mathcal{C}$;

ii) if $\sigma \in \Gamma$, then every face of $\sigma$ is in $\Gamma$;

iii) $\sigma_{i} \cap \sigma_{j}$ is a common face of $\sigma_{i}$ and $\sigma_{j}$.

It is proved that a pointed convex polyhedral cone $\mathcal{C}$ possesses a triangulation $\Gamma$ whose 1 -dim elements are the extreme rays of $\mathcal{C}$. We will always consider these triangulations in the following.

We are mainly interested in positive points in a pointed convex polyhedral cone $\mathcal{C}$. Define $E:=\mathcal{C} \cap \mathbb{N}^{d}$, resp. $\bar{E}:=\mathcal{C} \cap \mathbb{P}^{d}$. Their generating functions are computed using triangulations. The boundary of $\mathcal{C}$, denoted $\partial \mathcal{C}$, is the union of all the facets of $\mathcal{C}$. If $\Gamma$ is a triangulation of $\mathcal{C}$, let $\partial \Gamma$ denote the set $\{\sigma \in \Gamma, \sigma \in \partial \mathcal{C}\}$, and $\bar{\Gamma}=\Gamma \backslash \partial \Gamma$. Let $\sigma \in \Gamma$ be a simplicial cone, we set $E_{\sigma}:=\sigma \cap \mathbb{N}^{d}$ and $\bar{E}_{\sigma}:=\left\{v \in E_{\sigma}: v \notin E_{\tau}, \forall \tau \subset \sigma\right\}$. Then $\bar{E}$ is the disjoint union $\cup_{\sigma \in \bar{\Gamma}} \bar{E}_{\sigma}$ and

$$
\Phi_{\bar{E}}(\mathbf{x})=\sum_{\sigma \in \bar{\Gamma}} \Phi_{\bar{E}_{\sigma}}(\mathbf{x}) .
$$

Therefore to compute generating functions of cones, we compute generating functions of simplicial cones. Let $\mathbf{a}_{1}, \ldots, \mathbf{a}_{t}$ be a set of linearly independent integer vectors which generate the cone

$$
F:=\left\{\boldsymbol{\lambda} \in \mathbb{N}^{d}: n \boldsymbol{\lambda}=\lambda_{1} \mathbf{a}_{1}+\ldots+\lambda_{t} \mathbf{a}_{t}, n \in \mathbb{N}, \lambda_{i} \in \mathbb{N}\right\},
$$

$\mathbf{a}_{1}, \ldots, \mathbf{a}_{t}$ will be called a set of quasi generators of $F$. Define the interior $\bar{F}$ of $F$ as

$$
\bar{F}:=\left\{\boldsymbol{\lambda} \in \mathbb{P}^{d}: n \boldsymbol{\lambda}=\lambda_{1} \mathbf{a}_{1}+\ldots+\lambda_{t} \mathbf{a}_{t}, n \in \mathbb{P}, \lambda_{i} \in \mathbb{P}\right\}
$$


Consider the finite set $\bar{D}_{F}:=\left\{\boldsymbol{\lambda} \in F: \boldsymbol{\lambda}=\lambda_{1} \mathbf{a}_{1}+\ldots+\lambda_{t} \mathbf{a}_{t}, 0<\lambda_{i} \leq 1\right\}$. For any $\boldsymbol{\lambda} \in \bar{F}$, there exist unique $\boldsymbol{\beta} \in \bar{D}_{F}$ and $\lambda_{1}, \ldots, \lambda_{t} \in \mathbb{N}$ such that $\boldsymbol{\lambda}=$ $\boldsymbol{\beta}+\lambda_{1} \mathbf{a}_{1}+\ldots+\lambda_{t} \mathbf{a}_{t}$. Then [31, Prop. 4.6.8] yields:

$$
\Phi_{\bar{F}}(\mathbf{x})=\frac{\left(\sum_{\boldsymbol{\beta} \in \bar{D}_{F}} \mathbf{x}^{\boldsymbol{\beta}}\right)}{\prod_{i=1}^{t}\left(1-\mathbf{x}^{\mathbf{a}_{i}}\right)} .
$$

Given $F$, there is a unique set $C F(\bar{F}):=\left\{\boldsymbol{\beta}_{1}, \ldots, \boldsymbol{\beta}_{t}\right\}$ of primitive quasi generators. We say that $\bar{F}$ is strictly generated by $\boldsymbol{\beta}_{1}, \ldots, \boldsymbol{\beta}_{t}$ and call

$$
G_{\bar{F}}:=\left\{\boldsymbol{\lambda} \in \mathbb{P}^{d}: \boldsymbol{\lambda}=\lambda_{1} \boldsymbol{\beta}_{1}+\ldots+\lambda_{t} \boldsymbol{\beta}_{t}, 0<\lambda_{i} \leq 1\right\}
$$

the fundamental set of $\bar{F}$.

If $\mathcal{C}$ is a pointed polyhedral cone and $\Gamma$ a triangulation of $\mathcal{C}$, from 31, Prop. 4.6.10], we know that $C F(\bar{E}):=\cup_{\sigma \in \bar{\Gamma}} C F\left(\bar{F}_{\sigma}\right)$ is the set of $\boldsymbol{\beta} \in \mathcal{C} \cap \mathbb{P}^{d}$, which lie on extreme rays of $\mathcal{C}$ such that $\boldsymbol{\beta} \neq n \boldsymbol{\beta}^{\prime}$ for some $n>1$ and $\boldsymbol{\beta}^{\prime} \in \mathcal{C} \cap \mathbb{P}^{d}$. Furthermore in [31, Theorem 4.6.11] it is proved that the rational function $\Phi_{\bar{E}}(\mathbf{x}) \in \mathbb{Q}[\mathbf{x}]\left[D(\mathbf{x})^{-1}\right]$ where

$$
D(\mathbf{x})=\prod_{\boldsymbol{\beta} \in C F(\bar{E})}\left(1-\mathbf{x}^{\boldsymbol{\beta}}\right) .
$$

The last result we need is the following. Let $\mathcal{C}$ be a pointed polyhedral cone, let $b_{1}, \ldots, b_{d} \in \mathbb{Z}$ such that for each $r \in \mathbb{N}$, the number $g(r)$ of points in $\bar{E}=\mathcal{C} \cap \mathbb{P}^{d}$ such that $b_{1} \mathbf{a}_{1}+\ldots+\mathbf{a}_{d} b_{d}=r$ is finite. Let $G(\lambda)=\sum_{r \in \mathbb{N}} g(r) \lambda^{r}$. Then

$$
G(\lambda)=\Phi_{\bar{E}}\left(\lambda^{b_{1}}, \ldots, \lambda^{b_{d}}\right)
$$

\subsection{Motivic zeta function and Newton polyhedra.}

Newton diagrams for series. Let $h=\sum_{\mathbf{n} \in \mathbb{N}^{d}} a_{\mathbf{n}} \mathbf{x}^{\mathbf{n}} \in k[[\mathbf{x}]]$ be a formal power series with $h(0)=0$. The support of $h$ is the set $\operatorname{supp}(h)=\left\{\mathbf{n} \in \mathbb{N}^{d}: a_{\mathbf{n}} \neq 0\right\}$. The Newton polyhedron $\Gamma(h)$ of $h$ is the convex hull in $\mathbb{R}_{+}^{d}$ of the set $\bigcup_{\mathbf{n} \in \operatorname{supp}(h)}\left(\mathbf{n}+\left(\mathbb{R}_{+}\right)^{d}\right)$. The Newton polytope or Newton diagram $N D(h)$ of $h$ is the union of all compact faces of $\Gamma(h)$; the set of all compact faces is denoted by $C F(h)$. The principal part of $h$ is the polynomial $\left.h\right|_{N D(h)}:=\sum_{\mathbf{n} \in N D(h)} a_{\mathbf{n}} x^{\mathbf{n}}$. For any $\tau \in C F(h)$ we denote by $h_{\tau}$ the polynomial $\sum_{\mathbf{n} \in \tau} a_{\mathbf{n}} x^{\mathbf{n}}$. The principal part of $h$ is called non-degenerated if for each closed proper face $\tau \in C F(h)$, the subscheme of $\mathbf{G}_{m, k}^{d}$ defined by

$$
\frac{\partial h_{\tau}}{\partial x_{1}}=\ldots=\frac{\partial h_{\tau}}{\partial x_{d}}=0
$$

is empty.

Newton diagrams for polynomials. Let $h: \mathbb{A}_{k}^{d} \rightarrow \mathbb{A}_{k}^{1}$ be a regular morphism, $h(\mathbf{x})=\sum_{\mathbf{n} \in \mathbb{N}^{d}} a_{\mathbf{n}} \mathbf{x}^{\mathbf{n}}$. The support of $h$ is the set $\operatorname{supp}(h)=\left\{\mathbf{n} \in \mathbb{N}^{d}: a_{\mathbf{n}} \neq 0\right\}$. The global Newton polytope $\Gamma_{\mathrm{gl}}(h)$ of $h$ is the convex hull in $\mathbb{R}_{+}^{d}$ of the set $\operatorname{supp}(h)$. The polynomial $h$ is called 0-non-degenerated if for each closed face $\tau \subset \Gamma_{\mathrm{gl}}(h)$, including $\tau=\Gamma_{\mathrm{gl}}(h)$, the subscheme of $\mathbf{G}_{m, k}^{d}$ defined by $h_{\tau}=0$ is smooth over $k$. 
The Newton polyhedron $\Gamma_{\infty}(h)$ of $h$ at infinity is the convex hull of $\operatorname{supp}(h) \cup\{0\}$. The polynomial $h$ is nondegenerated with respect to $\Gamma_{\infty}(h)$ if for every face $\tau$ of $\Gamma_{\infty}(h)$ (of any dimension), which does not contain the origin, the subscheme of $\mathbf{G}_{m, k}^{d}$ defined by

$$
\frac{\partial h_{\tau}}{\partial x_{1}}=\ldots=\frac{\partial h_{\tau}}{\partial x_{d}}=0
$$

is empty. If $k$ is algebraically closed then the subscheme of $\mathbf{G}_{m, k}^{d}$ defined by $h_{\tau}=0$ is smooth over $k$ if and only if $h_{\tau}, x_{1} \frac{\partial h_{\tau}}{\partial x_{1}}, \ldots, x_{d} \frac{\partial h_{\tau}}{\partial x_{d}}$ have no common zero on the torus $\mathbf{G}_{m, k}^{d}$.

Let $h \in k[[\mathbf{x}]]$ be a formal power series and let $\omega$ be a regular differential form such that $(h, \omega)$ verifies the support condition [2.3, Assume that $h(\mathbf{x})=\prod_{j=1}^{d} x_{j}^{N_{j}} f(\mathbf{x})$, $N_{j} \in \mathbb{N}$, where $x_{j}$ does not divide $f$ for any $j=1, \ldots, d$, and the form $\omega$ equals $\left(\prod_{j=1}^{d} x_{j}^{\nu_{j}-1}\right) d x_{1} \wedge \ldots \wedge d x_{d}, \nu_{j} \geq 1$.

We recall more known definitions and properties. For $\mathbf{k}=\left(k_{1}, \ldots, k_{d}\right) \in \mathbb{R}_{+}^{d}$, we define $m_{h}(\mathbf{k}):=\inf _{\mathbf{x} \in \Gamma(h)}\{\mathbf{k} \cdot \mathbf{x}\}$ and $\sigma_{\omega}(\mathbf{k}):=\nu_{1} k_{1}+\ldots+\nu_{d} k_{d}$. Since $h$ is obtained from $f$ multiplying by a monomial then $\Gamma(h)$ is a translation of $\Gamma(f)$. In particular $m_{h}(\mathbf{k})=m_{f}(\mathbf{k})+N_{1} k_{1}+\ldots+N_{d} k_{d}$.

The first meet locus of $\mathbf{k} \in \mathbb{R}_{+}^{d}$ is $F(\mathbf{k}):=\left\{\mathbf{x} \in \Gamma(h): \mathbf{k} \cdot \mathbf{x}=m_{h}(\mathbf{k})\right\}$. If $\tau$ is a face of $\Gamma(h)$ (or $\Gamma(f))$ the cone associated with $\tau$ is the convex polyhedral cone, in the dual space, defined by $\Delta_{\tau}:=\left\{\mathbf{k} \in \mathbb{R}_{+}^{d}: F(\mathbf{k})=\tau\right\}$.

It is well-known that the cones associated with the elements of $C F(h)$ give a partition of (the dual space) $\mathbb{R}_{>0}^{d}$ in a disjoint union $\bigcup_{\tau \in C F(h)} \Delta_{\tau}$. It turns out that for each $\mathbf{k}=\left(k_{1}, \ldots, k_{d}\right) \in \mathbb{P}^{d}$ there exists a unique compact face $\tau$ such that $\mathbf{k} \in \Delta_{\tau}$. Given $\tau \in C F(h)$ we define, see [10] for a similar definition in the $p$-adic case,

$$
S_{\Delta_{\tau}}(h, \omega, T):=\sum_{\mathbf{k} \in \mathbb{P}^{d} \cap \Delta_{\tau}} \mathbb{L}^{-\sigma_{\omega}(\mathbf{k})} T^{m_{h}(\mathbf{k})} .
$$

Using the recalled results on generating functions, in particular (3.3), one has

$$
S_{\Delta_{\tau}}(h, \omega, T)=\Phi_{\mathbb{P}^{d} \cap \Delta_{\tau}}\left(\mathbb{L}^{-\nu_{1}} T^{p_{1}}, \ldots, \mathbb{L}^{-\nu_{d}} T^{p_{d}}\right),
$$

where $\left(p_{1}, \ldots, p_{d}\right) \in \tau$, for instance one of its vertices. In what follows we write $\Phi_{\Delta_{\tau}}(\mathbf{x}):=\Phi_{\mathbb{P}^{d} \cap \Delta_{\tau}}(\mathbf{x})$. The term $S_{\Delta_{\tau}}(h, \omega, T)$ can be computed as follows. Take a partition of the cone $\Delta_{\tau}$ into rational simplicial cones $\Delta_{i}, i=1, \ldots, s$, then $S_{\Delta_{\tau}}(h, \omega, T)=\sum_{i=1}^{s} S_{\Delta_{i}}(h, \omega, T)$. If $\Delta_{i}$ is the cone strictly generated by linearly independent vectors $\mathbf{a}_{1}, \ldots, \mathbf{a}_{r} \in \mathbb{N}^{d}$ then (3.1) implies

$$
S_{\Delta_{i}}(h, \omega, T)=\left(\sum_{\mathbf{g} \in G_{i}} \mathbb{L}^{-\sigma_{\omega}(\mathbf{g})} T^{m_{h}(\mathbf{g})}\right) \prod_{j=1}^{r} \frac{1}{1-\mathbb{L}^{-\sigma_{\omega}\left(\mathbf{a}_{j}\right)} T^{m_{h}\left(\mathbf{a}_{j}\right)}},
$$

where $G_{i}$ is the fundamental set of $\Delta_{i} \cap \mathbb{P}^{d}$ :

$$
G_{i}:=\mathbb{N}^{d} \cap\left\{\sum_{j=1}^{r} \mu_{j} \mathbf{a}_{j} \mid 0<\mu_{j} \leq 1 \text { for } j=1, \ldots, r\right\} .
$$


The multiplicity mult $\left(\Delta_{i}\right)$ of $\Delta_{i}$ is the cardinality of $G_{i}$. It is also equal to the volume of the parallelepiped spanned by $\mathbf{a}_{1}, \ldots, \mathbf{a}_{r}$ with respect to the volume form $\tilde{\omega}$ on the vector space $V$ generated by $\left\{\mathbf{a}_{1}, \ldots, \mathbf{a}_{r}\right\}$ normalized such that the parallelepiped spanned by a lattice basis of $\mathbb{Z}^{d} \cap V$ has volume 1 . We define

$$
J_{\Delta_{i}}(h, \omega, s):=\frac{\operatorname{mult}\left(\Delta_{i}\right)}{\prod\left(\sigma_{\omega}\left(\mathbf{a}_{j}\right)+m_{h}\left(\mathbf{a}_{j}\right) s\right)} .
$$

If $\Delta_{\tau}$ is a $r$-dimensional rational convex cone and $\Delta_{\tau}=\cup \Delta_{i}$ is a decomposition in rational simplicial cones $\Delta_{i}$ of dimension $r$ such that $\operatorname{dim}\left(\Delta_{i} \cap \Delta_{j}\right)<r$ for $i \neq j$, then we define $J_{\Delta_{\tau}}(h, \omega, s):=\sum J_{\Delta_{i}}(h, \omega, s)$. In fact $J_{\Delta_{\tau}}(h, \omega, s)$ is nothing but $J_{\Delta_{\tau}}(h, \omega, s)=\chi_{\text {top }}\left((\mathbb{L}-1)^{r} S_{\Delta}\left(h, \omega, \mathbb{L}^{-s}\right)\right)$ and it does not depend on the decomposition.

The following lemma follows from (3.2), (3.6) and the main theorem in 9 .

Lemma 3.1. $S_{\Delta_{\tau}}(h, \omega, T) \in \mathbb{Z}\left[\mathbb{L}, \mathbb{L}^{-1},\left(1-\mathbb{L}^{-\sigma_{\omega}(\mathbf{a})} T^{m_{h}(\mathbf{a})}\right)^{-1}\right][T]$, where a belongs to the set of vectors such that $\mathbf{a} \cdot \mathbf{x}=M$ is a reduced integral equation of an affine hyperplane containing $\tau \in C F(h)$.

We denote by $N_{\tau}$ the subvariety of $\mathbf{G}_{m, k}^{d}$ defined by $\left\{h_{\tau}=0\right\}$. The symbol $\left[N_{\tau}\right]$ means its class in the ring $K_{0}\left(\operatorname{Var}_{k}\right)$. Define $L_{\tau}^{A}(h):=\mathbb{L}^{-d}\left((\mathbb{L}-1)^{d}-\left[N_{\tau}\right]\right) \in \mathcal{M}_{k}$. Since $\Gamma(h)$ is a translation of $\Gamma(f)$, there exists a natural bijection between $C F(h)$ and $C F(f)$. Thus we can also write $\left[N_{\tau}\right]=\left[\mathbf{G}_{m, k}^{d} \cap\left\{f_{\tau}=0\right\}\right]$. Let $\tau \in C F(h)$ with $\operatorname{dim}(\tau)=d-r^{\prime}, 1 \leq r^{\prime} \leq d$.

If $r^{\prime}=d$, it means $\tau$ is a vertex of $\Gamma(h)$, then $\left[N_{\tau}\right]=0, \Delta_{\tau}$ is a $d$-dimensional rational convex polyhedron and $L_{\tau}^{A}(h)=\mathbb{L}^{-d}(\mathbb{L}-1)^{d}$. In such a case

$$
\chi_{\text {top }}\left(L_{\tau}^{A}(h) S_{\Delta_{\tau}}\left(h, \omega, \mathbb{L}^{-s}\right)\right)=\chi_{\text {top }}\left((\mathbb{L}-1)^{d} S_{\Delta_{\tau}}\left(h, \omega, \mathbb{L}^{-s}\right)\right)=J_{\Delta_{\tau}}(h, \omega, s) .
$$

If $r^{\prime} \in\{1, \ldots, d-1\}$, then $h_{\tau}(\mathbf{x})$ is a weighted homogeneous polynomial with more than one monomial. The quasi-projective variety $W:=\mathbb{A}_{k}^{d} \backslash\left\{h_{\tau}(\mathbf{x})=0\right\}$ is $k$-isomorphic to the affine algebraic variety $Y:=\left\{(\mathbf{x}, z) \in \mathbb{A}_{k}^{d} \times \mathbb{A}_{k}^{1}: z h_{\tau}(\mathbf{x})=1\right\}$. Under such an isomorphism, $W \cap \mathbf{G}_{m, k}^{d}$ is isomorphic to $Y \cap \mathbf{G}_{m, k}^{d+1}$. It implies that $\left[N_{\tau}\right]=\left[\mathbf{G}_{m, k}^{d}\right]-\left[W \cap \mathbf{G}_{m, k}^{d}\right]=(\mathbb{L}-1)^{d}-\left[Y \cap \mathbf{G}_{m, k}^{d+1}\right]$ in $K_{0}\left(\operatorname{Var}_{k}\right)$. Thus $L_{\tau}^{A}(h)=\mathbb{L}^{-d}\left[Y \cap \mathbf{G}_{m, k}^{d+1}\right]$.

Lemma 3.2 (see e.g. [10]). Let $g \in k\left[x_{1}, \ldots, x_{d}\right]$. If $d-r^{\prime}:=\operatorname{dim} \Gamma_{\infty}(g)<d$ then there exists a coordinate change $T$ on the torus $\mathbf{G}_{m, k}^{d}$ such that $g(\mathbf{x})=\left(g \circ T^{-1}\right)(\mathbf{y})=$ $\tilde{g}\left(y_{1}, \ldots, y_{d-r^{\prime}}\right)$, where $\tilde{g} \in k\left[y_{1}, \ldots, y_{d-r^{\prime}},\left(y_{1} \ldots y_{d-r^{\prime}}\right)^{-1}\right]$.

Moreover if $\tau$ is a face of $\Gamma_{\infty}(g)$ then $T^{-1}(\tau)$ is a face of $\tilde{g}$ and $g$ is non-degenerated with respect to $\Gamma_{\infty}(g)$ if and only if $\tilde{g}$ is non-degenerated with respect to $\Gamma_{\infty}(\tilde{g})$

In our case $g_{\tau}:=z h_{\tau}(\mathbf{x})$ is a weighted homogeneous polynomial whose Newton polyhedron has dimension $d-r^{\prime}+1<d+1$. Applying Lemma 3.2 we find a homogeneous polynomial $\tilde{g}_{\tau}(\mathbf{y})$ in $d-r^{\prime}+1$ variables such that the variety $Y \cap \mathbf{G}_{m, k}^{d+1}$ is $k$-isomorphic to $\mathbf{G}_{m, k}^{r^{\prime}} \times G^{*}$ where $G_{\tau}^{*}=\left\{\mathbf{y} \in \mathbf{G}_{m, k}^{d-r^{\prime}+1}: \tilde{g}_{\tau}(\mathbf{y})=1\right\}$. It turns 
out that $\mathbb{L}^{d} L_{\tau}^{A}(h)=\left[Y \cap \mathbf{G}_{m, k}^{d+1}\right]=(\mathbb{L}-1)^{r^{\prime}}\left[G_{\tau}^{*}\right]$ in $K_{0}\left(\operatorname{Var}_{k}\right)$. The convex rational rational cone $\Delta_{\tau}$ has dimension $r^{\prime}$, thus

$$
\chi_{\text {top }}\left(L_{\tau}^{A}(h) S_{\Delta_{\tau}}\left(h, \omega, \mathbb{L}^{-s}\right)\right)=\chi_{\text {top }}\left(\left[G_{\tau}^{*}\right]\right) J_{\Delta_{\tau}}(h, \omega, s) .
$$

Later on we will consider germs degenerated with respect to their Newton polyhedra. In the following definition we collect the terms corresponding to the $A$-part in the decomposition of the arc space according to these polyhedra.

Definition 3.3. Let $h \in k[[\mathbf{x}]]$ be a power series and $\omega$ a differential form such that $(h, \omega)$ satisfies condition (2.3). The $A$-part or the part corresponding to the Newton polyhedron of $h$ is defined by the rational function

$$
Z_{D L}^{A}(h, \omega, T):=\sum_{\tau \in C F(h)} L_{\tau}^{A}(h) S_{\Delta_{\tau}}(h, \omega) \in \mathcal{M}_{k}\left[\left(1-\mathbb{L}^{-\sigma_{\omega}(\mathbf{a})} T^{m_{h}(\mathbf{a})}\right)^{-1}\right][T],
$$

where $\mathbf{a}$ belongs to the set of vectors such that $\mathbf{a} \cdot \mathbf{x}=M$ is a reduced integer equation of an affine hyperplane containing some $\tau \in C F(h)$. In the same way we define the $A$-part of $(h, \omega)$ for the local topological zeta function as the rational function

$$
Z_{\mathrm{top}, 0}^{A}(h, \omega, s)=\sum_{\tau \in C F(h)} \chi_{\mathrm{top}}\left(\left[G_{\tau}^{*}\right]\right) J_{\Delta_{\tau}}(h, \omega, s),
$$

where $\chi_{\text {top }}\left(\left[G_{\tau}^{*}\right]\right):=1$ if $\operatorname{dim}(\tau)=0$.

If $h$ has non-degenerated Newton principal part, $Z_{D L}(h, \omega, T)$ is written in terms of some invariants of $C F(h)$ and $\omega$. This formula has been proved by J. Denef and $\mathrm{K}$. Hoornaert in the $p$-adic setting, [10. Our proof is based on their results, so we follow their notation too. In fact we provide the proof because it will give some light in the arc decomposition that we have to do in the quasi-ordinary case. G. Guibert has communicated to the authors that he has recently obtained a similar formula, 20].

Theorem 3.4. Let $h(\mathbf{x})=\prod_{j=1}^{d} x_{j}^{N_{j}} f(\mathbf{x}), N_{j} \in \mathbb{N}$, be a regular function with $h$ nondegenerated with respect to (all the compact faces of) its Newton polyhedron and let $\omega=\prod_{j=1}^{d} x_{j}^{\nu_{j}-1} d x_{1} \wedge \ldots \wedge d x_{d}, \nu_{j} \geq 1$ be a differential form verifying the support condition [2.3. Then

$$
Z_{D L}(h, \omega, T)=\sum_{\tau \in C F(h)} L_{\tau}(h) S_{\Delta_{\tau}}(h, \omega, T)
$$

where $L_{\tau}(h):=L_{\tau}^{A}(h)+L_{\tau}^{B}(h)$ and $L_{\tau}^{B}(h):=\mathbb{L}^{-d}(\mathbb{L}-1)\left[N_{\tau}\right] \frac{\mathbb{L}^{-1} T}{1-\mathbb{L}^{-1} T}$.

We break the proof in several steps.

Step 1. Classifying arcs.

Let $S$ be the affine hypersurface $x_{1} \cdots x_{d}=0$. Since $S$ has dimension less than $d$ then by property $(b)$ of the motivic measure the set $\mathcal{L}_{0}(S)$ has measure zero. Then we only consider $\operatorname{arcs} \varphi \in \mathcal{L}_{0}\left(\mathbb{A}_{k}^{d}\right) \backslash \mathcal{L}_{0}(S)$, i.e., $\boldsymbol{\varphi}=\left(\varphi_{1}, \ldots, \varphi_{d}\right)$ where $\varphi_{i}(t)=$ 
$a_{k_{i}} t^{k_{i}}+$ higher degree terms. As usual we write $\mathbf{k}(\boldsymbol{\varphi}):=\left(k_{1}, \ldots, k_{d}\right) \in \mathbb{P}^{d}$ and $\mathbf{a}(\boldsymbol{\varphi}):=\left(a_{k_{1}}, \ldots, a_{k_{d}}\right) \in \mathbf{G}_{m, k}^{d}$.

Let $\tau$ be the unique compact face of $N D(h)$ such that $\mathbf{k}=\mathbf{k}(\boldsymbol{\varphi}) \in \Delta_{\tau} \cap \mathbb{P}^{d}$. If $\mathbf{a}=\mathbf{a}(\boldsymbol{\varphi}) \in \mathbf{G}_{m, k}^{d} \backslash N_{\tau}$ then $\operatorname{ord}(h \circ \boldsymbol{\varphi})=\operatorname{ord}\left(h_{\tau} \circ \boldsymbol{\varphi}\right)=m_{h}(\mathbf{k})$. Otherwise, if $\mathbf{a} \in N_{\tau}$ then $\operatorname{ord}(h \circ \boldsymbol{\varphi})>m_{h}(\mathbf{k})$.

The set $\bar{V}_{n, m}=\left\{\boldsymbol{\varphi} \in \mathcal{L}_{0}\left(\mathbb{A}_{k}^{d}\right) \backslash \mathcal{L}_{0}(S): \operatorname{ord}(h \circ \boldsymbol{\varphi})=n\right.$, ord $\left.(\omega \circ \boldsymbol{\varphi})=m\right\}$ can be decomposed as $\bar{V}_{n, m}=\bigcup_{\tau \in C F(f)}\left(V_{n, A, m}^{\tau} \cup V_{n, B, m}^{\tau}\right)$, where

$$
\begin{gathered}
V_{n, A, m}^{\tau}:=\left\{\boldsymbol{\varphi} \in \bar{V}_{n, m}: \mathbf{k}(\boldsymbol{\varphi}) \in \Delta_{\tau}, \mathbf{a}(\boldsymbol{\varphi}) \in \mathbf{G}_{m, k}^{d} \backslash N_{\tau}\right\}, \\
V_{n, B, m}^{\tau}:=\left\{\boldsymbol{\varphi} \in \bar{V}_{n, m}: \mathbf{k}(\boldsymbol{\varphi}) \in \Delta_{\tau}, \mathbf{a}(\boldsymbol{\varphi}) \in N_{\tau}\right\} .
\end{gathered}
$$

Rewrite the formula (2.2) as:

$$
\begin{aligned}
Z_{D L}(h, w, T) & =\sum_{\tau \in C F(h)}\left(\sum_{n \geq 1}\left(\sum_{m \in \mathbb{P}} \mathbb{L}^{-m} \mu_{X}\left(V_{n, A, m}^{\tau}\right)\right) T^{n}+\right. \\
& \left.+\sum_{n \geq 1}\left(\sum_{m \in \mathbb{P}} \mathbb{L}^{-m} \mu_{X}\left(V_{n, B, m}^{\tau}\right)\right) T^{n}\right) .
\end{aligned}
$$

Step 2. Computation of the $A$-part of the series.

Let $\tau \in C F(h)$ and $\mathbf{k} \in \Delta_{\tau}$. Define

$$
V_{A}^{\mathbf{k}}:=\left\{\boldsymbol{\varphi} \in \mathcal{L}_{0}\left(\mathbb{A}_{k}^{d}\right) \backslash \mathcal{L}_{0}(S): \mathbf{k}(\boldsymbol{\varphi})=\mathbf{k}, f_{\tau}(\mathbf{a}(\boldsymbol{\varphi})) \neq 0\right\}
$$

Given $\boldsymbol{\varphi} \in V_{A}^{\mathbf{k}}$ then $\operatorname{ord}(h \circ \boldsymbol{\varphi})$ and $\operatorname{ord}(\omega \circ \boldsymbol{\varphi})$ depend only on $\mathbf{k}$, therefore $V_{A}^{\mathbf{k}} \subset$ $V_{n_{\mathbf{k}}, A, m_{\mathbf{k}}}^{\tau}$ where $n_{\mathbf{k}}:=m_{h}(\mathbf{k})=m_{f}(\mathbf{k})+N_{1} k_{1}+\ldots N_{d} k_{d}$ and $m_{\mathbf{k}}:=\sigma_{\omega}(\mathbf{k})-\left(k_{1}+\right.$ $\left.\cdots+k_{d}\right)$. The stable semialgebraic set $V_{A}^{\mathbf{k}}$ has measure $\mu_{X}\left(V_{A}^{\mathbf{k}}\right)=\left[\pi_{n_{\mathbf{k}}}\left(V_{A}^{\mathbf{k}}\right)\right] \mathbb{L}^{-\left(n_{\mathbf{k}}+1\right) d}$. Since

$$
\left[\pi_{n_{\mathbf{k}}}\left(V_{A}^{\mathbf{k}}\right)\right]=\left[\mathbf{G}_{m, k}^{d} \backslash\left\{f_{\tau}=0\right\}\right] \mathbb{L}^{d n_{\mathbf{k}}-k_{1}-\ldots-k_{d}}
$$

then $\mathbb{L}^{-m_{\mathbf{k}}} \mu_{X}\left(V_{A}^{\mathbf{k}}\right)=\mathbb{L}^{-d}\left((\mathbb{L}-1)^{d}-\left[N_{\tau}\right]\right) \mathbb{L}^{-\sigma_{\omega}(\mathbf{k})}$.

It turns out that

$$
\begin{array}{r}
\sum_{n \geq 1}\left(\sum_{m \in \mathbb{N}} \mathbb{L}^{-m} \mu_{X}\left(V_{n, A, m}^{\tau}\right)\right) T^{n}=\sum_{\mathbf{k} \in \mathbb{P}^{d} \cap \Delta_{\tau}} \mathbb{L}^{-m_{\mathbf{k}}} \mu_{X}\left(V_{A}^{\mathbf{k}}\right) T^{n_{\mathbf{k}}}= \\
=\sum_{\mathbf{k} \in \mathbb{P}^{d} \cap \Delta_{\tau}} \mathbb{L}^{-d}\left((\mathbb{L}-1)^{d}-\left[N_{\tau}\right]\right) \mathbb{L}^{-\sigma_{\omega}(\mathbf{k})} T^{m(\mathbf{k})+N_{1} k_{1}+\ldots N_{d} k_{d}}= \\
=\mathbb{L}^{-d}\left((\mathbb{L}-1)^{d}-\left[N_{\tau}\right]\right) \sum_{\mathbf{k} \in \mathbb{P}^{d} \cap \Delta_{\tau}} \mathbb{L}^{-\sigma_{\omega}(\mathbf{k})} T^{m_{h}(\mathbf{k})}=L_{\tau}^{A}(h) S_{\Delta_{\tau}}(h, \omega, T) .
\end{array}
$$

Step 3. Computation of the $B$-part of the series.

Let $\tau \in C F(h)$. As before for any $\mathbf{k} \in \mathbb{P}^{d} \cap \Delta_{\tau}$ we define the sets $V_{n, B}^{\mathbf{k}}$ of $\operatorname{arcs} \boldsymbol{\varphi}$ in $V_{n, B}^{\tau}$ such that $\mathbf{k}(\boldsymbol{\varphi})=\mathbf{k}$. Note that $V_{n, B}^{\mathbf{k}} \subset V_{n, B, m_{\mathbf{k}}}^{\tau}$. 
Lemma 3.5. Fix $\mathbf{k} \in \mathbb{P}^{d} \cap \Delta_{\tau}$ as before. Then

$$
\sum_{n \geq 1} \mathbb{L}^{-m_{\mathbf{k}}} \mu_{X}\left(V_{n, B}^{\mathbf{k}}\right) T^{n}=\mathbb{L}^{-d}(\mathbb{L}-1)\left[N_{\tau}\right] \frac{\mathbb{L}^{-1} T}{1-\mathbb{L}^{-1} T} \mathbb{L}^{-\sigma_{\omega}(\mathbf{k})} T^{m_{h}(\mathbf{k})} .
$$

The formula in Theorem 3.4 is deduced from the following equality which is a consequence of the above lemma:

$$
\sum_{\mathbf{k} \in \mathbb{P}^{d} \cap \Delta_{\tau}} \sum_{n \geq 1} \mathbb{L}^{-m_{\mathbf{k}}} \mu_{X}\left(V_{n, B}^{\mathbf{k}}\right) T^{n}=\mathbb{L}^{-d}(\mathbb{L}-1)\left[N_{\tau}\right] \frac{\mathbb{L}^{-1} T}{1-\mathbb{L}^{-1} T} S_{\Delta_{\tau}}(h, \omega, T) .
$$

Proof of Lemma 3.5. Given $\mathbf{k} \in \mathbb{P}^{d} \cap \Delta_{\tau}$, consider the $k[t]$-morphism $\pi_{\mathbf{k}}: Y \rightarrow \mathbb{A}_{k[t]}^{d}$ defined by $\pi_{\mathbf{k}}\left(y_{1}, \ldots, y_{d}\right)=\left(t^{k_{1}} y_{1}, \ldots, t^{k_{d}} y_{d}\right)$, where $Y:=\mathbb{A}_{k}^{d}$. Let $\boldsymbol{\varphi} \in V_{n, B}^{\mathbf{k}}$ be an arc, then the equalities $\varphi_{i}(t)=t^{k_{i}} \psi_{i}(t), i=1, \ldots, d$, define a unique arc $\boldsymbol{\psi}(t):=$ $\left(\psi_{1}(t), \ldots, \psi_{d}(t)\right) \in \mathcal{L}(Y)$ centered at $\boldsymbol{\psi}(0)=\mathbf{a}(\boldsymbol{\varphi}) \in \mathbf{G}_{m, k}^{d} \cap\left\{f_{\tau}=0\right\}$. It verifies $\operatorname{ord}\left(f \circ \pi_{\mathbf{k}} \circ \boldsymbol{\psi}\right)=\operatorname{ord}(f \circ \boldsymbol{\varphi})=n$ and because of the quasi-homogeneity of $f_{\tau}$ :

$$
f \circ \pi_{\mathbf{k}}\left(y_{1}, \ldots, y_{d}\right)=t^{m(\mathbf{k})}\left(f_{\tau}\left(y_{1}, \ldots, y_{d}\right)+t g\left(y_{1}, \ldots, y_{d}\right)\right),
$$

where the function $g$ has coefficients in $k[t]$. In particular $n=m_{f}(\mathbf{k})+n^{\prime}$ with $n^{\prime}:=\operatorname{ord}\left(f_{1} \circ \boldsymbol{\psi}\right) \geq 1$ where $f_{1}\left(y_{1}, \ldots, y_{d}\right):=f_{\tau}\left(y_{1}, \ldots, y_{d}\right)+t g\left(y_{1}, \ldots, y_{d}\right)$. The algebraic set $f_{\tau}\left(y_{1}, \ldots, y_{d}\right)=0$ is nonsingular at points of $\mathbf{G}_{m, k}^{d} \cap\left\{f_{\tau}=0\right\}$ because of the non-degeneracy condition. In particular at any of such points there are coordinates such that $f_{\tau}=\bar{y}_{1}$ and in the same way at such a point $f_{1}=0$ will be nonsingular. Applying the change variables formula the proof of the lemma is finished. Remark that the pull-back by $\pi_{\mathbf{k}}$ of the regular differential form $d x_{1} \wedge \ldots \wedge d x_{d}$ is $t^{\sigma_{\omega}(\mathbf{k})} d y_{1} \wedge \ldots \wedge d y_{d}$.

A formula for $Z_{\text {top }, 0}(h, \omega, s)$ for a non-degenerated function $h$ was given in [12. We apply Theorem 3.4 to reprove that formula. We need several standard definitions.

Definition 3.6. Let $\gamma$ be the convex hull in $\mathbb{R}^{d}$ of some subset of $\mathbb{Z}^{d}$. The volume form $\omega_{\gamma}$ on the affine space $\operatorname{Aff}(\gamma)$ generated by $\gamma$ is defined such that the parallelepiped generated by a base of the lattice $\operatorname{Aff}(\gamma) \cap \mathbb{Z}^{d}$ has volume 1 . For any $\tau \in C F(h), V(\tau)$ is the volume of $\tau$ relative to the induced lattice; i.e. to the volume form $\omega_{\tau}$.

Theorem 3.7. 12] If $h$ has non-degenerated principal part and the pair $(h, \omega)$ verifies the support condition (2.3) then

$$
\begin{aligned}
Z_{\mathrm{top}, 0}(h, \omega, s)= & \sum_{\tau \in C F(h), \operatorname{dim} \tau=0} J_{\Delta_{\tau}}(h, \omega, s) \\
& \quad+\left(\frac{s}{s+1}\right) \sum_{\tau \in C F(h), \operatorname{dim} \tau \geq 1}(-1)^{\operatorname{dim}(\tau)} \operatorname{dim}(\tau) ! V(\tau) J_{\Delta_{\tau}}(h, \omega, s) .
\end{aligned}
$$


The proof follows the same ideas as in equations (3.8) and (3.9). Let $\tau \in C F(h)$. If $\tau$ is zero dimensional we have the identity (3.8). Otherwise, following the same notation as in equation (3.9), if $\operatorname{dim}(\tau)=d-r^{\prime}$ then

$$
L_{\tau}(h)=\mathbb{L}^{-d}\left((\mathbb{L}-1)^{d+1} \frac{\mathbb{L}^{-1} T}{1-\mathbb{L}^{-1} T}+(\mathbb{L}-1)\left[Y \cap \mathbf{G}_{m, k}^{d+1}\right] \frac{1-T}{1-\mathbb{L}^{-1} T}\right) .
$$

Since $d-r^{\prime}<d$, the denominator of $S_{\Delta_{\tau}}(h, \omega, T)$ has at most $d-1$ factors and

$$
\chi_{\text {top }}\left(\mathbb{L}^{-d}(\mathbb{L}-1)^{d+1} \frac{\mathbb{L}^{-1} T}{1-\mathbb{L}^{-1} T} S_{\Delta_{\tau}}\left(h, \omega, \mathbb{L}^{-s}\right)\right)=0 .
$$

Therefore we have again the term $\left[Y \cap \mathbf{G}_{m, k}^{d+1}\right]$. Applying Lemma 3.2 to $g_{\tau}:=z h_{\tau}(\mathbf{x})$ we have $\left[Y \cap \mathbf{G}_{m, k}^{d+1}\right]=(\mathbb{L}-1)^{r^{\prime}}\left[G_{\tau}^{*}\right]$ in $K_{0}\left(\operatorname{Var}_{k}\right)$ where $G_{\tau}^{*}=\left\{\mathbf{y} \in \mathbf{G}_{m, k}^{d-r^{\prime}+1}: \tilde{g}_{\tau}(\mathbf{y})=1\right\}$ for some non-degenerated weighted homogeneous polynomial $\tilde{g}_{\tau}(\mathbf{y})$. Finally we also need the following result.

Lemma 3.8. 4, 11] Let $g\left(y_{1}, \ldots, y_{n}\right)$ be a 0-non-degenerated polynomial and let $Z^{*}=\left\{y \in \mathbf{G}_{m, k}^{n}: g(\mathbf{y})=0\right\}$. Then the $\ell$-adic Euler characteristic $\chi_{\mathrm{top}}\left(Z^{*}\right)$ is given by

$$
\chi_{\text {top }}\left(Z^{*}\right)=(-1)^{n-1} n ! \operatorname{Vol}_{n}(\Delta(g)) .
$$

Here $\operatorname{Vol}_{n}(\Delta(g))$ is the $n$-dimensional Euclidean volume.

In our case the polynomial $g=\tilde{g}_{\tau}-1$ is 0 -non-degenerated and verifies the hypothesis of the Lemma 3.8, then $\chi_{\text {top }}\left(G^{*}\right)=(-1)^{r}(r+1)$ ! $\operatorname{Vol}_{r+1}(\Delta(g))$. Since $\Delta(g)$ is a cone over the origin, $(r+1)$ ! $\operatorname{Vol}_{r+1}(\Delta(g))=r ! V(\tau)$ (this last volume as in definition 3.6). Hence we get the formula in Theorem 3.7 .

Remark 3.9. All the results in this section are also valid in the complex analytic set up.

Example 3.10. It is clear that the poles of $Z_{\text {top }, 0}(h, \omega, s)$ come from the poles of $Z_{D L}(h, \omega, T)$ but in general the other way around is not true. Consider for instance $h=x_{1}^{3}+x_{2}^{3}+x_{3}^{3}+x_{4}^{3}+x_{5}^{6}$ and the differential form $\omega=d \mathbf{x}$. This example has been recently studied by Ishii-Kollar in [23] to disprove a J. Nash conjecture.

Then $h$ has non-degenerated principal part and its Newton polyhedron has only one compact facet $\tau$ which, in the dual, corresponds with the extreme ray $v_{\tau}:=$ $(2,2,2,2,1)$. Take the vertex $(3,0,0,0,0)$ of the face $\tau$, then

$$
S_{\Delta_{\tau}}(h, \omega, T)=\Phi_{\Delta_{\tau}}\left(\mathbb{L}^{-1} T^{3}, \mathbb{L}^{-1} T^{0}, \mathbb{L}^{-1} T^{0}, \mathbb{L}^{-1} T^{0}, \mathbb{L}^{-1} T^{0}\right)=\frac{\mathbb{L}^{-9} T^{6}}{1-\mathbb{L}^{-9} T^{6}} .
$$

After Lemma 3.1. $\left(1-\mathbb{L}^{-9} T^{6}\right)$ is a possible factor of the denominator of $Z_{D L}(h, \omega, T)$. In fact it is case. To prove it, one considers the Igusa $p$-adic zeta function of $h$ and apply [10, Theorem 5.17] which guarantees that $t_{0}=-3 / 2$ is one of its poles for $p \gg 0$. Nevertheless the local topological zeta function is $Z_{\text {top }, 0}(h, \omega, s)=1 /(1+s)$. This shows an example where poles of $p$-adic and local topological zeta function are not the same. 


\section{QUASI-ORDINARY POWER SERIES}

In this section we recall some known properties of the quasi-ordinary power series. We give the necessary details to describe the method we will use to compute their local zeta functions. Let $k$ be an algebraically closed field of characteristic zero.

Let $h \in k[[\mathbf{x}]][z]$ be a $z$-polynomial of degree $s$ with coefficients in the formal power series $k[[\mathbf{x}]], \mathbf{x}=\left(x_{1}, \ldots, x_{d}\right)$. Assume that $h(\mathbf{x}, z)=x_{1}^{N_{1}} \ldots x_{d}^{N_{d}} g(\mathbf{x}, z)$ where $N_{l} \geq 0$ and $x_{l}$ doesn't divide $g(\mathbf{x}, z), \forall l, l=1, \ldots, d$, . Let's write the decomposition of $g(\mathbf{0}, z), g(\mathbf{0}, z)=u \prod\left(z-z_{b}\right)^{s_{b}}$, with $s=\sum s_{b}, u \in \mathbf{G}_{m, k}$ and $z_{b} \in \mathbb{A}_{k}^{1}$.

The power series $h$ is called quasi-ordinary (or $k$-quasi-ordinary if we want to emphasize the base field) if its $z$-discriminant $D_{z}(g)$ (or equivalently $\left.D_{z}(h)\right)$ is

$$
D_{z}(g)=x_{1}^{\alpha_{1}} x_{2}^{\alpha_{2}} \ldots x_{d}^{\alpha_{d}} \varepsilon(\mathbf{x}),
$$

where $\varepsilon(\mathbf{0}) \neq 0$. The condition on the $z$-discriminant implies that $g(\mathbf{x}, z)$ is squarefree in the ring $k[[\mathbf{x}, z]]$.

For each root $z_{b}$ of $g(\mathbf{0}, z)$, the Jung-Abhyankar Theorem, [1, 27], states that there are exactly $s_{b}$ distinct roots of $g(\mathbf{x}, z)$ centred at $z_{b}$ in $k\left[\left[x_{1}^{1 / m_{b}}, \ldots, x_{d}^{1 / m_{b}}\right]\right]$, for some $m_{b} \in \mathbb{P}$. Thus for each $j \in\left\{1, \ldots, s_{b}\right\}$, there exists a fractional power series $\zeta_{b_{j}} \in k\left[\left[x_{1}^{1 / m_{b}}, \ldots, x_{d}^{1 / m_{b}}\right]\right]$ with $\zeta_{b_{j}}(\mathbf{0})=z_{b}$ such that

$$
g(\mathbf{x}, z)=\prod_{b} \prod_{j=1}^{s_{b}}\left(z-\zeta_{b_{j}}\left(x_{1}^{1 / m_{b}}, \ldots, x_{d}^{1 / m_{b}}\right)\right) .
$$

In fact, for each root $z_{b}$, the product $\prod_{j=1}^{s_{b}}\left(z-\zeta_{b_{j}}\left(x_{1}^{1 / m_{b}}, \ldots, x_{d}^{1 / m_{b}}\right)\right)$ is a well defined element in $k[[\mathbf{x}]][z]$. Moreover, this power series is quasi-ordinary too because of the properties of the discriminant.

In principle we are interested just in one of the roots, say $z_{b}=0$, otherwise we can a translation of type $\bar{z}=z-z_{b}$ to study the corresponding root. Later on when the transversal sections will be described we will deal with other roots different from $z_{b}=0$, see section 4.5 .

Therefore we may assume

$$
h(\mathbf{x}, z)=x_{1}^{N_{1}} \ldots x_{d}^{N_{d}} f(\mathbf{x}, z) u(\mathbf{x}, z),
$$

where $f(\mathbf{x}, z)$ is a degree $n$ quasi-ordinary $z$-Weierstrass polynomial, $n$ being the multiplicity of the root $z_{b}=0$ in $g(\mathbf{0}, z), u(\mathbf{x}, z)$ is a unity in $k[[\mathbf{x}, z]], u(\mathbf{x}, z)$ being the product of all other roots centred at points different from $z_{b}=0$, and $N_{l} \geq 0$ for any $l=1, \ldots, d$. Therefore, for each $j \in\{1, \ldots, n\}$, there exist $\zeta_{j} \in$ $k\left[\left[x_{1}^{1 / m}, \ldots, x_{d}^{1 / m}\right]\right]$ (for some positive integer $m$ ) with $\zeta_{j}(\mathbf{0})=0$ such that

$$
f(\mathbf{x}, z)=\prod_{j=1}^{n}\left(z-\zeta_{j}\left(x_{1}^{1 / m}, \ldots, x_{d}^{1 / m}\right)\right) .
$$


Let $\zeta$ be a root of $f$, all its conjugates $\tilde{\zeta}_{\varepsilon}:=\zeta\left(\varepsilon_{1} x_{1}^{1 / m}, \ldots, \varepsilon_{d} x_{d}^{1 / m}\right)$, where $\varepsilon_{l}^{m}=1$, for all $l=1, \ldots, d$, are also roots of $f$. If $f$ is irreducible in $k[[\mathbf{x}]][z]$, then all its roots are conjugate to one of them, say $\zeta$, and the set $\left\{\tilde{\zeta}_{\varepsilon}\right\}$ has exactly $n$ distinct elements $\left\{\zeta=\zeta_{1}, \ldots, \zeta_{n}\right\}$ which verify

$$
f(\mathbf{x}, z)=\prod_{p=1}^{n}\left(z-\zeta_{p}\right) .
$$

The irreducible factors of $f$ in $k[[\mathbf{x}]][z]$ are also $k$-quasi-ordinary power series. Let $\left\{f^{(i)}\right\}_{i \in I}$ be the set of irreducible factors of $f$. Then

$$
f(\mathbf{x}, z)=\prod_{i \in I} \prod_{j=1}^{n^{(i)}}\left(z-\zeta_{j}^{(i)}\right)
$$

The set of indexes $I$ is decomposed in two disjoint subsets: $I^{\prime}$ and $I \backslash I^{\prime}$ such that

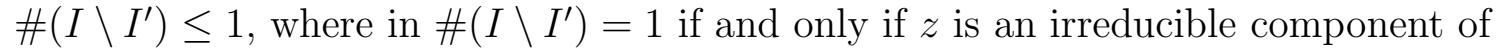
$f$ (i.e. if 0 is root of $f$ ).

Definition 4.1. We will say that a variable $x_{i}$ is essential for $h$ if $x_{i}$ divides $D_{z}(h)$. The number of essential variables will be denoted by $\mathbf{e v}(h)$.

Remark 4.2. All our results for the local Denef-Loeser motivic zeta function of quasi-ordinary power series are proved in the case where $g$ is reduced. Nevertheless they can be proved in the nonreduced case. This means that $g$ can have multiple components in $k[[\mathbf{x}, z]]$. In such a case the power series $h(\mathbf{x}, z)$ will be called quasiordinary if $x_{1}^{N_{1}} \ldots x_{d}^{N_{d}} g_{\text {red }}(\mathbf{x}, z)$ is quasi-ordinary in the above sense.

We are only interested in the local Denef-Loeser motivic zeta function. Since for any $\operatorname{arc} \varphi(t)$, with $\boldsymbol{\varphi}(\mathbf{0})=0$, the $t$-order $\operatorname{ord}(h \circ \boldsymbol{\varphi})$ does not depend on the unity $u(\mathbf{x}, z)$ then we usually suppose that $u(\mathbf{x}, z)=1$.

\subsection{Characteristic exponents.}

Given $h(\mathbf{x}, z)=\prod_{l=1}^{d} x_{l}^{N_{l}} f(\mathbf{x}, z)=\prod_{l=1}^{d} x_{l}^{N_{l}} \prod_{i \in I}\left(z-\zeta_{i}\right)$, one has $D_{z}(f)=\prod\left(\zeta_{k}-\right.$ $\zeta_{j}$ ). For two different roots $\zeta_{k}$ and $\zeta_{j}$ of $f$, using the unique factorization of the discriminant, we have

$$
\zeta_{k}-\zeta_{j}=x_{1}^{\lambda_{k j, 1}} x_{2}^{\lambda_{k j, 2}} \ldots x_{d}^{\lambda_{k j, d}} \varepsilon_{k j}\left(x_{1}^{1 / m}, \ldots, x_{d}^{1 / m}\right),
$$

where $\varepsilon_{k j}(0) \neq 0$, and $\lambda_{k j, t} \in \frac{1}{m} \mathbf{z}_{\geq 0}$.

Definition 4.3. We set $\Lambda_{C E}(f):=\left\{\boldsymbol{\lambda}_{k j}\right\}=\left\{\left(\lambda_{k j, 1}, \ldots, \lambda_{k j, d}\right)\right\}_{k j} \subset \frac{1}{m} \mathbf{z}_{\geq 0}^{d}$. The elements of the finite set $\Lambda_{C E}(f)$ are called characteristic exponents of $f$. We usually identify $\Lambda_{C E}(h)$ with $\Lambda_{C E}(f)$ because $\Lambda_{C E}(f)$ is the set of characteristic exponents of $h$ at the root zero. We usually omit "at the root zero" whenever it will be clear.

For each characteristic exponent $\boldsymbol{\lambda}=\left(\lambda_{1}, \ldots, \lambda_{d}\right)$, the corresponding monomial $\mathrm{x}^{\boldsymbol{\lambda}}$ is called characteristic monomial of $f$. In the same way we identify characteristic monomials of $h$ at zero with characteristic monomials of $f$. 
Since the irreducible factors $f^{(i)}$ of $f$ are also quasi-ordinary, the set of characteristic exponents $\Lambda_{C E}\left(f^{(i)}\right)$ verifies $\Lambda_{C E}\left(f^{(i)}\right) \subset \Lambda_{C E}(f)$ for every $i \in I$. The other characteristic exponents of $f$ measure the order of coincidence between distinct irreducible factors of $f$ (as in the case of plane curves).

The following partial ordering will be used in the paper. For any $\boldsymbol{\lambda}, \boldsymbol{\mu} \in \mathbb{Q}^{d}$, then $\boldsymbol{\lambda} \leq \boldsymbol{\mu}$ if $\lambda_{l} \leq \mu_{l}$ for all $l=1, \ldots, d$. We say that $\boldsymbol{\lambda}<\boldsymbol{\mu}$ if $\boldsymbol{\lambda} \leq \boldsymbol{\mu}$ and there is an $l$ such that $\lambda_{l}<\mu_{l}$. If $f(\mathbf{x}, z)$ is irreducible in $k[[\mathbf{x}, z]]$, then the elements of $\Lambda_{C E}(f)$ are totally ordered, see [24] and the references therein. But this is no longer true in the non-irreducible case.

Up to know to have the same characteristic exponents is just that two sets coincide. Later on, we will give a new definition of having the same characteristic exponents (which will extend the usual definition for curves).

\subsection{Newton polyhedron and good coordinates.}

In this section we show that for every quasi-ordinary power series $h(\mathbf{x}, z)$ there exist coordinates, called good coordinates, such that the compact faces of $N D(h)$ have only dimension 1 and 0 . This means that the 1-dimensional compact faces of $N D(h)$ are totally ordered by their slopes and they form a monotone polygonal path. Our definition is slightly more general than the definition of good coordinates given by P. González in [18, [19, Lemma 3.16]; we will call them P-good coordinates. He proves the existence of $P$-good coordinates for quasi-ordinary power series; in fact his $P$-good coordinates are a particular case of our good coordinates, see Remark 4.8. One of the main properties of $P$-good coordinates is that they are uniquely defined, which is not the case for good coordinates. The advantage of these last ones is that they are preserved when passing to transversal sections, see subsection 4.5. this is not the case for $P$-good coordinates.

Definition 4.4. A quasi-ordinary power series $h \in k[[\mathbf{x}]][z]$ is in good coordinates if $N D(h)$ is a monotone polygonal path such that either $z$ is a component of $h$ or $h_{\gamma}$ is not the product of a monomial in $\mathbf{x}$ and a power of a linear form, where $\gamma$ is the compact 1-dimensional face of $N D(h)$ which meets the plane $z=0$, i.e. $h_{\gamma} \neq x_{1}^{a_{1}} \ldots x_{d}^{a_{d}}\left(z-\alpha x_{1}^{b_{1}} \ldots x_{d}^{b_{d}}\right)^{m}$ for all $\alpha \in \mathbf{G}_{m, k}$.

Proposition 4.5. There exists a system of coordinates which are good for $h$.

Proof. Since $h$ and $f$ essentially differ by a monomial, the Newton polyhedron $\Gamma(h)$ is obtained from $\Gamma(f)$ by the translation induced by the corresponding monomial. Thus it is enough to prove the result for $f$ being a Weierstrass quasi-ordinary polynomial of degree $n$. We proceed by induction on $n$. The case $n=1$ is clear: we have $f=z+a(\mathbf{x})$, with $a(\mathbf{x}) \in k[[\mathbf{x}]]$, and the change $z_{1}=z+a(\mathbf{x})$ is enough.

In order to proceed by induction we need the following notion introduced by Hironaka and studied in [27.

Definition 4.6. Let $f(\mathbf{x}, z)=z^{n}+a_{n-1}(\mathbf{x}) z^{n-1}+\ldots+a_{0}(\mathbf{x})$ be a quasi-ordinary Weierstrass polynomial, set $\tau_{0}=(0, \ldots, 0, n)$ the corresponding vertex in $N D(f)$. We say that $f$ is $\nu$-quasi-ordinary with respect to $z$ if there is a vertex $\tau_{1}$ in $N D(f)$, 
$\tau_{1} \neq \tau_{0}$, such that if $\tau$ is the projection of $\tau_{1}$ over $\mathbb{N}^{d} \times\{0\}$ from $\tau_{0}$ and $\gamma$ is the segment joining $\tau_{0}$ and $\tau$ then $N D(f)$ is contained in $\cup_{\mathbf{n} \in \gamma}\left(\mathbf{n}+\mathbb{R}_{+}^{d+1}\right)$ and $f_{\gamma}$ is a polynomial which is not a power of a linear form.

In [27] it is proved that if we make the change $z=z_{1}-\frac{a_{n-1}(\mathbf{x})}{n}$ the polynomial $f$ becomes $\nu$-quasi-ordinary. If $N D(f)=\gamma$ (with the above notations) then $\gamma$ is the only 1-dimensional compact face, therefore the last one, and $f_{\gamma}$ is not a power of a linear form so we are done. Otherwise, in [27] it is shown that $f$ can be decomposed as a product $f=f_{0}(\mathbf{x}, z) f_{1}(\mathbf{x}, z), f_{i}(\mathbf{x}, z) \in k[[\mathbf{x}]][z]$, such that $f_{\gamma}$ corresponds with the factor $f_{0}$, recall that the Newton polytope of $f$ is the Minkowski sum of the Newton polytopes of each factor. The factor $f_{1}$ is a quasi-ordinary Weierstrass polynomial of degree less than $n$ and we apply induction to conclude. In fact the change of variables we need for the induction step are of type $z_{1}=z_{2}+b(\mathbf{x})$ and they do not affect the first segment of Newton polytope of $f$ because of the condition $N D(f)$ is contained in $\cup_{\mathbf{n} \in \gamma}\left(\mathbf{n}+\mathbb{R}_{+}^{d+1}\right)$ in the definition of $\nu$-quasi-ordinary.

Remark 4.7. Observe that from the proof of the proposition 4.5 one gets that it is enough to make change of variables of type $z=z_{1}+a(\mathbf{x}), a(\mathbf{x}) \in k[[\mathbf{x}]]$ to get good coordinates. The procedure to get good coordinates is far from being unique. For instance $f(x, z)=\left(z^{2}-x^{5}\right)\left(z-x^{2}\right)\left(z^{3}-x^{2}\right)$ is already in good coordinates but the described method gives us different good coordinates.

Let $h$ be a quasi-ordinary power series in good coordinates. Since $N D(h)$ is a monotone polygonal path we order the set $\{\gamma\}$ of compact 1-dimensional faces according with the slopes (which are rational $d$-tuples) of the edges using the partial order defined before. In fact they are totally ordered and the smallest one will be the $z$-highest. Assume that there are exactly $r$ of them which are ordered as $\gamma_{1}, \ldots, \gamma_{r}$. For $q \in\{1, \ldots, r\}$, since $\gamma_{q}$ is 1-dim, the weighted-homogeneous polynomial $f_{\gamma_{q}}=$ $z^{k} \prod x_{l}^{a_{l}}\left(z^{n_{1}^{q} m_{q}}+\ldots+\alpha\left(x_{1}^{b_{1}^{q}} \ldots x_{d}^{b_{d}^{q}}\right)^{m_{q}}\right)$, for some non negative integers $k, a_{l}$ and $m_{q}:=\operatorname{gcd}\left(n_{1}^{q} m_{q}, b_{1}^{q} m_{q}, \ldots, b_{d}^{q} m_{q}\right)$. Since $\gamma_{q}$ is an edge of the Newton polytope then $n_{1}^{q}$ is a positive integer, the $d$-uple $\left(b_{1}^{q}, \ldots, b_{d}^{q}\right) \in \mathbb{N}^{d}$ is non-zero and $\alpha \in \mathbf{G}_{m, k}$. The polynomial $f\left(\mathbf{1}, z^{1 / n_{1}^{q}}\right)$ of degree $m_{q}$ in $k[z]$ can be factorized. There are some positive integers $m_{q, j}, j=1, \ldots, v(q)$, such that $f_{\gamma_{q}}=z^{k} \prod x_{l}^{a_{l}} f_{\boldsymbol{\lambda}_{q}}$ where

$$
f_{\boldsymbol{\lambda}_{q}}:=\prod_{j=1}^{v(q)}\left(f_{q, j}\right)^{m_{q, j}}, \text { where } f_{q, j}:=z^{n_{1}^{q}}-\beta_{j}^{q} \mathbf{x}^{n_{1}^{q} \boldsymbol{\lambda}_{q}}=z^{n_{1}^{q}}-\beta_{j}^{q} x_{1}^{b_{1}^{q}} \ldots x_{d}^{b_{d}^{q}},
$$

$\lambda_{q, l}=\frac{b_{l}^{q}}{n_{1}^{q}}$ for each $l=1, \ldots, d$. We define the rational $d$-uple $\boldsymbol{\lambda}_{q}:=\left(\lambda_{q, 1}, \ldots, \lambda_{q, d}\right) \in$ $\mathbb{Q}^{d}$. We will say that $f_{\boldsymbol{\lambda}_{q}}$ is the weighted-homogeneous polynomial associated with the compact 1-dimensional face $\gamma_{q}$ of $\Gamma(f)$.

Remark 4.8. Let us see that we can find good coordinates which are not $P$-good coordinates in the example $f(x, z)=\left(z^{2}-x^{5}\right)\left(z-x^{2}\right)\left(z^{3}-x^{2}\right)$. The problem arises in the characteristic exponents which are integers. The definition-construction of $P$-good coordinates shows that there exists a change of coordinates of type $z_{1}=$ 
$z+a(\mathbf{x}), a(\mathbf{x}) \in k[[\mathbf{x}]]$, such that in the set $\Lambda_{N D}(h):=\left\{\boldsymbol{\lambda}_{1}, \ldots, \boldsymbol{\lambda}_{r}\right\}$, the greatest characteristic exponent $\boldsymbol{\lambda}_{r}$ is the unique which can be in $\mathbb{Z}^{d}$; moreover, its Newton initial form $f_{\boldsymbol{\lambda}_{r}}$ cannot be a power of a linear form. In the above example doing the change $z_{1}=z+2 x^{2}$ we get a new Newton polytope which is a polygonal path with only two 1-dimensional faces. We observe two facts. The first one is that for each quasi-ordinary power series in good coordinates, the method described in [19. Lemma 3.16] provides P-good coordinates. The second one is that in any case, the characteristic exponents appearing in both Newton polyhedra which are smaller than the first integer characteristic exponent coincide in both polytopes. Our constructions work in $P$-good coordinates but proofs must be slightly modify.

Proposition 4.9. If $h$ is in good coordinates then each $\boldsymbol{\lambda}_{q}$ is a characteristic exponent of $f$.

Proof. Since $h$ is in good coordinates we can use the process given in [27] to compute the roots of $f$ from the Newton polytope. In fact each root, different from zero, is found in only one of the edges of $N D(h)$. Assume $\gamma_{1}, \ldots, \gamma_{r}$ are the edges ordered as before. Then any pair $\zeta \in \gamma_{q}$ and $\zeta^{\prime} \in \gamma_{q+1}$ gives $\boldsymbol{\lambda}_{q}$ as characteristic exponent, that is $\zeta-\zeta^{\prime}=\mathbf{x}^{\boldsymbol{\lambda}_{q}} \epsilon(\mathbf{x}), \epsilon(\mathbf{0}) \neq 0$. If $\gamma_{q}=\gamma_{r}$, as $h$ is in good coordinates, either $z$ is a factor of $h$ and $\zeta-0=\mathbf{x}^{\boldsymbol{\lambda}_{r}}, \zeta \in \gamma_{r}$ or $f_{\gamma_{r}}$ is not a power of a linear form and taking two distinct roots in $\gamma_{r}$ gives $\boldsymbol{\lambda}_{r}$.

Thus each root $\zeta_{i}$ of $f$ is written in a unique way as

$$
\zeta_{i}(\mathbf{x})=\alpha_{1}^{(i)} \mathbf{x}^{\boldsymbol{\lambda}_{(i)}}+\sum_{\boldsymbol{\lambda}_{(i)}<\boldsymbol{\lambda}} \alpha_{\boldsymbol{\lambda}}^{(i)} \mathbf{x}^{\boldsymbol{\lambda}},\left(\alpha_{1}^{(i)} \neq 0\right) .
$$

We define $f_{q}$ to be the product of all roots $\zeta$ of $f$ whose initial term gives the Newton polytope of $f_{\boldsymbol{\lambda}_{q}}$. This means that

$$
f_{q}(\mathbf{x}, z):=\prod_{\operatorname{roots}: \boldsymbol{\lambda}_{(i)}=\boldsymbol{\lambda}_{q}}\left(z-\zeta_{i}\right)
$$

Remark 4.10. Since all the conjugates under the Galois group of a root $\zeta_{i}$ have the same $\boldsymbol{\lambda}_{(i)}$, the irreducible component in $k[[\mathbf{x}]][z]$ of $f(\mathbf{x}, z)$ determined by $\zeta_{i}$ divides $f_{q}(\mathbf{x}, z)$. Thus $f_{q} \in k[[\mathbf{x}]][z]$ because it is a product of some irreducible components of $f(\mathbf{x}, z)$.

Thus we define the set $\Lambda_{N D}(h):=\left\{\boldsymbol{\lambda}_{1}, \ldots, \boldsymbol{\lambda}_{r}\right\}$ which is a subset of the characteristic exponents of $h$ and which are ordered $\boldsymbol{\lambda}_{r}>\ldots>\boldsymbol{\lambda}_{1}$.

Let $f_{j}^{q}$ be the element in $k[[\mathbf{x}]][z]$ defined by

$$
f_{j}^{q}(\mathbf{x}, z):=\prod_{\operatorname{roots}: \boldsymbol{\lambda}_{(i)}=\boldsymbol{\lambda}_{q} \text { and } \beta^{(i)}=\beta_{j}^{q}}\left(z-\zeta_{i}\right) .
$$


The same ideas as in Remark 4.10 show that $f_{j}^{q} \in k[[\mathbf{x}]][z]$. Hence

$$
f_{q}=\prod_{j=1}^{v(q)} f_{j}^{q} \quad \text { and } \quad f= \begin{cases}\prod_{q=1}^{r} \prod_{j=1}^{v(q)} f_{j}^{q}, & \text { if } I=I^{\prime} \text { and } \\ z \prod_{q=1}^{r} \prod_{j=1}^{v(q)} f_{j}^{q}, & \text { otherwise. }\end{cases}
$$

Definition 4.11. If $h$ is in good coordinates then we call $\left\{f_{j}^{q}\right\}_{q, j}$ the Newton components of $f$; each $f_{j}^{q}$ defines a quasi-ordinary power series.

\subsection{Dual decomposition.}

Assume a pair $(h, \omega)$ verifying the support condition 2.3 is given, where $h$ is a quasi-ordinary power series in good coordinates as in (4.2) and $\omega=\prod_{j=1}^{d} x_{j}^{\nu_{j}-1} d x_{1} \wedge$ $\ldots \wedge d x_{d} \wedge d z, \nu_{j} \geq 1$. The compact faces of $\Gamma(f)$ (or $\left.\Gamma(h)\right)$ are 1-dim edges $\gamma_{1}, \ldots, \gamma_{r}$ with their corresponding vertices $\tau_{0}, \tau_{1}, \ldots, \tau_{r}$.

Remark 4.12. The main point in the Newton polytope is that its set of compact faces (and then its vertices) is totally ordered. In principle they are ordered by the order imposed to the characteristic exponents. From now on, if we do not emphasize the contrary, we assume they are ordered with the reverse order that is such that $\tau_{r}$ has the highest $z$-coordinate.

Let $e_{1}, \ldots, e_{d+1}$ denote the canonical basis of the dual space $\left(\mathbb{R}^{d+1}\right)^{*}$ where we choose coordinates $\left(v_{1}, \ldots, v_{d}, v_{d+1}\right)$. The fan $\Sigma(\Gamma(h)) \subset\left(\mathbb{R}^{d+1}\right)^{*}$ is obtained subdividing the cone $\left(\mathbb{R}_{+}^{d+1}\right)^{*}$ with linear hyperplanes $l^{q}: \eta_{q}=0$ where

$$
\eta_{q}:=\sum_{l=1}^{d} b_{l}^{q} v_{l}-n_{1}^{q} v_{d+1}=n_{1}^{q}\left(\sum_{l=1}^{d} \lambda_{\kappa_{q, l}} v_{l}-v_{d+1}\right), q=1, \ldots, r .
$$

Let $\Delta_{\gamma_{q}}=\left\{\mathbf{k} \in \mathbb{R}_{+}^{d+1} \mid \mathbf{k} \cdot\left(b_{1}^{q}, \ldots, b_{d}^{q},-n_{1}^{q}\right)=0\right\}$ be its dual cone.

Lemma 4.13. $\Delta_{\gamma_{q}}$ is the strictly positive simplicial cone $\Delta_{\gamma_{q}}=\left\{\lambda_{1} \mathbf{w}_{1}^{q}+\ldots+\right.$ $\left.\lambda_{d} \mathbf{w}_{d}^{q}, \lambda_{i} \in \mathbb{R}_{+}\right\}$, where $\mathbf{w}_{l}^{q}:=\frac{1}{c_{l}^{q}}\left(n_{1}^{q} e_{l}+b_{l}^{q} e_{d+1}\right) \in \mathbb{N}^{d+1}, c_{l}^{q}:=\operatorname{gcd}\left(n_{1}^{q}, b_{l}^{q}\right), \bar{b}_{l}^{q}:=$ $\frac{b_{l}^{q}}{c_{l}^{q}}, p_{l}^{q}:=\frac{n_{1}^{q}}{c_{l}^{q}}$ with $l=1 \ldots, d$.

The linearly independent vectors $\left\{\mathbf{w}_{l}^{q}\right\}_{l=1, . . d}$ are primitive, so the proof is clear. Let $G_{q}$ be the fundamental set of $\Delta_{\gamma_{q}}$, then (3.1) implies

$$
\Phi_{\Delta_{\gamma_{q}}}(\mathbf{y}):=\Phi_{\Delta_{\gamma_{q}} \cap \mathbb{P}^{d+1}}(\mathbf{y})=\frac{\sum_{\boldsymbol{\beta} \in G_{q}} \mathbf{y}^{\boldsymbol{\beta}}}{\prod_{l=1}^{d}\left(1-\mathbf{y}^{\mathbf{w}_{l}^{q}}\right)} .
$$

If $p=\left(p_{1}, \ldots, p_{d}, p_{d+1}\right)$ is an element in the closure of $\gamma_{q}$ then (3.5) gives

$$
S_{\Delta_{\gamma_{q}}}(h, \omega, T)=\Phi_{\Delta_{\gamma_{q}}}\left(\mathbb{L}^{-\nu_{1}} T^{p_{1}}, \ldots, \mathbb{L}^{-\nu_{d+1}} T^{p_{d+1}}\right) .
$$

The edge $\gamma_{q}$ is defined by the affine equations $n_{1}^{q} x_{l}+b_{l}^{q} z=M_{l}^{q}, \quad l=1, \ldots, d$, for some positive integers $M_{l}^{q}$. 
Lemma 4.14. $S_{\Delta_{\gamma_{q}}}(h, \omega, T) \in \mathbb{Z}\left[\mathbb{L}, \mathbb{L}^{-1},\left(1-\mathbb{L}^{-\left(\nu_{l} p_{l}^{q}+\bar{b}_{l}^{q}\right)} T^{\frac{M_{l}^{q}}{c_{l}^{q}}}\right)^{-1}\right][T], l \in\{1, \ldots, d\}$ and

$$
J_{\gamma_{q}}(h, \omega, s)=\frac{\left(n_{1}^{q}\right)^{d-1}}{\tilde{T}_{1}^{q} \ldots \tilde{T}_{d}^{q}}
$$

where $\tilde{T}_{l}^{q}:=\left(M_{l}^{q} s+\nu_{l} n_{1}^{q}+b_{l}^{q}\right), l=1, \ldots, d$ and $q=1, \ldots, r$.

Proof. Since $m_{h}\left(\mathbf{w}_{l}^{q}\right)=\mathbf{w}_{l}^{q} \cdot \mathbf{a}$, where $\mathbf{a} \in \gamma_{q}$, then $m_{h}\left(\mathbf{w}_{l}^{q}\right)=\frac{M_{l}^{q}}{c_{l}^{q}}$. On the other hand, $\sigma_{w}\left(\mathbf{w}_{l}^{q}\right)=\mathbf{w}_{l}^{q} \cdot\left(\nu_{1}, \ldots, \nu_{d}, 1\right)=\frac{1}{c_{l}^{q}}\left(\nu_{l} n_{1}^{q}+b_{l}^{q}\right)$. Let us compute $J_{\gamma_{q}}(h, \omega, s)$. Because of $\operatorname{gcd}\left(b_{1}^{q}, \ldots, b_{d}^{q},-n_{1}^{q}\right)=1$ we choose $\beta:=\left(a_{1}, \ldots, a_{l}, u\right)$ such that $\left(b_{1}^{q}, \ldots, b_{d}^{q},-n_{1}^{q}\right) \cdot \beta=$ 1. Computing $\operatorname{mult}\left(\Delta_{\gamma_{q}}\right)=\left|\operatorname{det}\left(\mathbf{w}_{1}^{q}, \ldots, \mathbf{w}_{d}^{q}, \beta\right)\right|$ by induction one can prove that

$$
\operatorname{det}\left(\mathbf{w}_{1}^{q}, \ldots, \mathbf{w}_{d}^{q}, \beta\right)=-\left(n_{1}\right)^{d-1} \frac{\left(b_{1}^{q}, \ldots, b_{d}^{q},-n_{1}^{q}\right) \cdot \beta}{\prod_{l=1}^{d} c_{l}^{q}}=-\frac{\left(n_{1}^{q}\right)^{d-1}}{\prod_{l=1}^{d} c_{l}^{q}} .
$$

The result follows from (3.7).

Consider next the first vertex $\tau_{0}$ with coordinates $\left(\mathbf{a}, d_{0}\right)$ in $\Gamma(h)$. The rational simplicial cone $\Delta_{\tau_{0}}$ is the $(d+1)$-dimensional cone: $\mathbb{R}_{+} \mathbf{w}_{1}^{1}+\cdots+\mathbb{R}_{+} \mathbf{w}_{d}^{1}+\mathbb{R}_{+} e_{d+1}$. In a similar manner as above if $G_{\tau_{0}}$ is the fundamental set of $\Delta_{\tau_{0}}$ then

$$
\Phi_{\Delta_{\tau_{0}}}(\mathbf{y})=\frac{\left(\sum_{\boldsymbol{\beta} \in G_{\tau_{0}}} \mathbf{y}^{\boldsymbol{\beta}}\right)}{\left(1-y_{d+1}\right) \prod_{l=1}^{d}\left(1-\mathbf{y}^{\mathbf{w}_{l}^{1}}\right)}
$$

and

$$
S_{\Delta_{\tau_{0}}}(h, \omega, T)=\Phi_{\Delta_{\tau_{0}}}\left(\mathbb{L}^{-\nu_{1}} T^{a_{1}}, \ldots, \mathbb{L}^{-\nu_{d}} T^{a_{d}}, \mathbb{L}^{-1} T^{d_{0}}\right) .
$$

Recall that $I=I^{\prime}$ if and only if $d_{0}=0$; otherwise $d_{0}=1$.

\section{Lemma 4.15.}

$$
S_{\Delta_{\tau_{0}}}(h, \omega, T) \in \mathbb{Z}\left[\mathbb{L}, \mathbb{L}^{-1},\left(1-\mathbb{L}^{-1} T\right)^{-\varepsilon},\left(1-\mathbb{L}^{-\left(\nu_{l} p_{1}^{1}+\bar{b}_{l}^{1}\right)} T^{\frac{M_{l}^{r}}{c_{l}^{1}}}\right)^{-1}\right][T]
$$

with $l \in\{1, \ldots, d\}$ and

$$
J_{\tau_{0}}(h, \omega, s)=\frac{\left(n_{1}^{1}\right)^{d}}{(s+1)^{\varepsilon} \tilde{T}_{1}^{1} \ldots \tilde{T}_{d}^{1}}, \quad \text { see Lemma 4.14, }
$$

where $\varepsilon=0$ or 1 accordingly with $I=I^{\prime}$ or not.

Proof. If $I=I^{\prime}, m_{h}\left(e_{d+1}\right)=0$ and $\sigma_{\omega}\left(e_{d+1}\right)=1$. Furthermore, $\operatorname{mult}\left(\Delta_{\tau_{0}}\right)=$ $\left|\operatorname{det}\left(\mathbf{w}_{1}^{1}, \ldots, \mathbf{w}_{d}^{1}, e_{d+1}\right)\right|=\frac{\left(n_{1}^{1}\right)^{d}}{\prod_{l=1}^{d} c_{l}^{1}}$, and we are done. The other case is similar.

Finally consider any other vertex $\tau_{q}$ of $\Gamma(h)$, different from $\tau_{0}$, which is the intersection of the edges $\gamma_{q}$ and $\gamma_{q+1}, q=1, \ldots, r$. If $q=r$ then $\gamma_{r+1}$ is a (non-compact) line parallel to $z$-axis. 
The dual cone $\Delta_{\tau_{q}}$ is $\sum_{i=1}^{d}\left(\mathbb{R}_{+} \mathbf{w}_{i}^{q}+\mathbb{R}_{+} \mathbf{w}_{i}^{q+1}\right)$, where if $q=r$ then $\mathbf{w}_{l}^{r+1}:=e_{l}$, for $l=1, \ldots, d$. For any $q=1, \ldots, r$, consider the strictly positive simplicial cone

$$
\Delta_{q}^{c}:=\sum_{l=1}^{d} \mathbb{R}_{+} \mathbf{w}_{l}^{q}+\mathbb{R}_{+} e_{d+1}
$$

For instance $\Delta_{r+1}^{c}$ is the whole positive cone $\mathbb{P}^{d+1}$. Moreover its multiplicity verifies

$$
\operatorname{mult}\left(\Delta_{q}^{c}\right)=\left|\operatorname{det}\left(\mathbf{w}_{1}^{q}, \ldots, \mathbf{w}_{d}^{q}, e_{d+1}\right)\right|=\frac{\left(n_{1}^{q}\right)^{d}}{\prod_{l=1}^{d} c_{l}^{q}} .
$$

The following relation holds for the indicator functions of these cones:

$$
\left[\Delta_{\tau_{q}}\right]-\left[\Delta_{q+1}^{c}\right]+\left[\Delta_{\gamma_{q}}\right]+\left[\Delta_{q}^{c}\right]=0, \quad q=1 \ldots, r,
$$

which implies the same relation among the corresponding generating functions. Since $\Delta_{q}^{c}$ and $\Delta_{\gamma_{q}}$ are simplicial cones,

$$
\Phi_{\Delta_{\tau_{q}}}(\mathbf{y})=\Phi_{\Delta_{q+1}^{c}}(\mathbf{y})-\left(\Phi_{\Delta_{\gamma_{q}}}(\mathbf{y})+\Phi_{\Delta_{q}^{c}}(\mathbf{y})\right) .
$$

If $G_{q}^{c}$ is the fundamental set of $\Delta_{q}^{c}$ then

$$
\begin{aligned}
\Phi_{\Delta_{\tau_{q}}}(\mathbf{y}) & =\frac{\left(\sum_{\boldsymbol{\beta} \in G_{q+1}^{c}} \mathbf{y}^{\boldsymbol{\beta}}\right)}{\left(1-y_{d+1}\right) \prod_{l=1}^{d}\left(1-\mathbf{y}^{\mathbf{w}_{l}^{q+1}}\right)}- \\
& -\left(\frac{\left(\sum_{\boldsymbol{\beta} \in G_{q}} \mathbf{y}^{\boldsymbol{\beta}}\right)}{\prod_{l=1}^{d}\left(1-\mathbf{y}^{\mathbf{w}_{l}^{q}}\right)}+\frac{\left(\sum_{\boldsymbol{\beta} \in G_{q}^{c}} \mathbf{y}^{\boldsymbol{\beta}}\right)}{\left(1-y_{d+1}\right) \prod_{l=1}^{d}\left(1-\mathbf{y}^{\mathbf{w}_{l}^{q}}\right)}\right) .
\end{aligned}
$$

If $\tau_{q} \in \Gamma(h)$ has $z$-height $d_{q}$ then

$$
S_{\Delta_{\tau_{q}}}(h, \omega, T)=\Phi_{\Delta_{\tau_{q}}}\left(\mathbb{L}^{-\nu_{1}} T^{a_{1}}, \ldots, \mathbb{L}^{-\nu_{d}} T^{a_{d}}, \mathbb{L}^{-1} T^{d_{q}}\right) .
$$

Hence since $m_{h}\left(e_{j}\right)=N_{j}$ and $\sigma_{\omega}\left(e_{j}\right)=\nu_{j}$ then

$$
S_{\Delta_{\tau_{r}}}(h, \omega, T) \in \mathbb{Z}\left[\mathbb{L}^{ \pm 1}, \frac{1}{1-\mathbb{L}^{-\nu_{j}} T^{N_{j}}}, \frac{1}{1-\mathbb{L}^{-\left(\nu_{l} p_{l}^{r}+\bar{b}_{l}^{r}\right)} T^{\frac{M_{l}^{r}}{c_{l}^{r}}}}\right][T],
$$

with $j, l \in\{1, \ldots, d\}$. In the same way, for each $q \in\{1, \ldots, r-1\}$,

$$
S_{\Delta_{\tau_{q}}}(h, \omega, T) \in \mathbb{Z}\left[\mathbb{L}^{ \pm 1}, \frac{1}{1-\mathbb{L}^{-\left(\nu_{l} p_{l}^{q}+\bar{b}_{l}^{q}\right)} T^{\frac{M_{l}^{q}}{c_{l}^{q}}}}, \frac{1}{1-\mathbb{L}^{-\left(\nu_{l} p_{l}^{q+1}+\bar{b}_{l}^{q+1}\right)} T^{\frac{M_{l}^{q+1}}{c_{l}^{q+1}}}}\right][T]
$$

with $l=1, \ldots, d$. 
To compute more explicitly the local topological zeta function we argue as follows. Recall that $J_{\tau_{q}}(h, \omega, s)$ can be computed using an adequate simplicial decomposition of $\Delta_{\tau_{q}}$. We use the decomposition from (4.11). Then

$$
J_{\tau_{q}}(h, \omega, s)=\chi_{\text {top }}\left((\mathbb{L}-1)^{d+1}\left(\Phi_{\Delta_{q+1}^{c}}(\mathbf{a})-\left(\Phi_{\Delta_{\gamma_{q}}}(\mathbf{a})+\Phi_{\Delta_{q}^{c}}(\mathbf{a})\right)\right)\right),
$$

where $\mathbf{a}:=\left(\mathbb{L}^{-\left(\nu_{1}+a_{1} s\right)}, \ldots, \mathbb{L}^{-\left(\nu_{d}+a_{d} s\right)}, \mathbb{L}^{-\left(1+d_{q} s\right)}\right)$. Since $\Phi_{\Delta_{\gamma_{q}}}(\mathbf{a})$ has at most $d$ poles then

$$
J_{\tau_{q}}(h, \omega, s)=\chi_{\text {top }}\left((\mathbb{L}-1)^{d+1}\left(\Phi_{\Delta_{q+1}^{c}}(\mathbf{a})-\Phi_{\Delta_{q}^{c}}(\mathbf{a})\right)\right) .
$$

Moreover we know from equation (4.9) that $\operatorname{mult}\left(\Delta_{q}^{c}\right)=\frac{\left(n_{1}^{q}\right)^{d}}{\prod_{l=1}^{d} c_{l}^{q}}$.

Corollary 4.16. For each $q \in\{1, \ldots, r-1\}$, then

$$
J_{\tau_{q}}(h, \omega, s)=\frac{1}{\left(1+d_{q} s\right)}\left(\frac{\left(n_{1}^{q+1}\right)^{d}}{\tilde{T}_{1}^{q+1} \ldots \tilde{T}_{d}^{q+1}}-\frac{\left(n_{1}^{q}\right)^{d}}{\tilde{T}_{1}^{q} \ldots \tilde{T}_{d}^{q}}\right),
$$

and if $q=r$ then

$$
J_{\tau_{r}}(h, \omega, s)=\frac{1}{\left(1+d_{r} s\right)}\left(\frac{1}{T_{1} \ldots T_{d}}-\frac{\left(n_{1}^{r}\right)^{d}}{\tilde{T}_{1}^{r} \ldots \tilde{T}_{d}^{r}}\right),
$$

where $T_{i}:=N_{i} s+\nu_{i}, \tilde{T}_{l}^{r}$ have been defined in Lemma 4.14, and $i, l \in\{1, \ldots, d\}$.

Remark 4.17. It follows from the above discussion, definition 3.3 and Remark 4.18 that

$$
\begin{gathered}
Z_{D L}^{A}(h, \omega, T)=\sum_{j=0}^{r} L_{\tau_{j}}^{A}(h) S_{\Delta_{\tau_{j}}}(h, \omega, T)+\sum_{q=1}^{r} L_{\gamma_{q}}^{A}(h) S_{\Delta_{\gamma_{q}}}(h, \omega, T)= \\
=\mathbb{L}^{-(d+1)}(\mathbb{L}-1)^{d+1}\left(\sum_{j=0}^{r} S_{\Delta_{\tau_{j}}}(h, \omega, T)+\sum_{q=1}^{r}\left(1-\frac{v(q)}{\mathbb{L}-1}\right) S_{\Delta_{\gamma_{q}}}(h, \omega, T)\right) \\
Z_{\text {top }, 0}^{A}(h, \omega, s)=\sum_{j=0}^{r} J_{\tau_{j}}(h, \omega, s)-\sum_{q=1}^{r} v(q) J_{\gamma_{q}}(h, \omega, s) .
\end{gathered}
$$

After Lemma 3.1] each $\left(1-\mathbb{L}^{-1} T^{d_{q}}\right)$ or $\left(1+d_{q} s\right)$ appearing in (4.12) and Corollary 4.16 do not contribute to the denominator of the $A$-part of the corresponding zeta functions of $(h, \omega)$.

\subsection{Newton map associated with a Newton component.}

We have already discussed the dual decomposition associated with the Newton polyhedra of quasi-ordinary power series in good coordinates. In general these power series are degenerated with respect to their Newton polyhedron. In this paragraph we describe how to improve a quasi-ordinary power series in terms of the complexity of the series. 
Each compact 1-dimensional face $\gamma_{q}$ of $\Gamma(f)$ corresponds to a polynomial

$$
f_{\boldsymbol{\lambda}_{q}}=\prod_{j=1}^{v(q)}\left(z^{n_{1}^{q}}-\beta_{j}^{q} \mathbf{x}^{n_{1}^{q} \boldsymbol{\lambda}_{q}}\right)^{m_{q, j}}=\prod_{j=1}^{v(q)}\left(z^{n_{1}^{q}}-\beta_{j}^{q} x_{1}^{b_{1}^{q}} \ldots x_{d}^{b_{d}^{q}}\right)^{m_{q, j}} .
$$

Fix $j \in\{1, \ldots, v(q)\}$, the corresponding polynomial $f_{q, j}=z^{n_{1}^{q}}-\beta_{j}^{q} \mathbf{x}^{n_{1}^{q} \boldsymbol{\lambda}_{q}}$ and the series $f_{j}^{q}$, see definition 4.11. We denote by $r \leq d$, the number of essential variables, $\mathbf{e v}\left(f_{q, j}\right)$, of the irreducible factor $f_{q, j}$. For the sake of simplicity we assume that $x_{1}, \ldots, x_{r}$ are the essential variables of $f_{q, j}$. Fix $\pi_{j}^{q}: \mathbb{A}_{k}^{r} \rightarrow V_{j}^{q} \subset \mathbb{A}_{k}^{r+1}$ a bijective morphism (a parametrization) of the irreducible variety defined by $f_{q, j}$ :

$$
\left(s_{1}, \ldots, s_{r}\right) \mapsto\left(s_{1}^{p_{1}^{q}}, \ldots, s_{r}^{p_{r}^{q}}, \alpha_{j}^{q} s_{1}^{\bar{b}_{1}^{q}} \ldots s_{r}^{\bar{b}_{d}^{r}}\right),
$$

where $\left(\alpha_{j}^{q}\right)^{n_{1}^{q}}=\beta_{j}^{q}$.

Remark 4.18. In $K_{0}\left(\operatorname{Var}_{k}\right)$, one has $\left[V_{j}^{q}\right]=\mathbb{L}^{r}$. Later on we will consider the subvariety $\left(V_{j}^{q} \times \mathbb{A}_{k}^{d-r}\right) \cap \mathbf{G}_{m, k}^{d+1}$ on the torus; its class in $K_{0}\left(\operatorname{Var}_{k}\right)$ is $(\mathbb{L}-1)^{d}$. Since $f_{\boldsymbol{\lambda}_{q}}$ has $v(q)$ irreducible components, $\left[N_{\gamma_{q}}\right]=v(q)(\mathbb{L}-1)^{d}$.

Definition 4.19. Let $X=\operatorname{Spec} k[[\mathbf{x}, z]], Y=\operatorname{Spec} k\left[\left[\mathbf{y}, z_{1}\right]\right]$. The Newton map associated with the Newton component $f_{j}^{q}$ (or with the polynomial $f_{q, j}$ ) of $f$ is the morphism $\pi_{q, j}: Y \rightarrow X, \pi_{q, j}\left(\mathbf{y}, z_{1}\right):=(\mathbf{x}, z)$ defined by the equations $x_{l}=y_{l}^{p_{l}^{q}}$ for every $l=1, \ldots, d$, and $z=\left(z_{1}+\alpha_{j}^{q}\right) \prod_{l=1}^{d} y_{l}^{\bar{b}_{l}^{q}}$.

The integers $\bar{b}_{l}^{q}, p_{l}^{q}$ were defined in Lemma 4.13 and if $l=r+1, \ldots, d$, then $\bar{b}_{l}^{q}:=0$ and $p_{l}^{q}:=1$.

Lemma 4.20. Let $h$ be a quasi-ordinary power series in good coordinates. The pullback $\bar{h}_{q, j}$ of $h$ under the Newton map $\pi_{q, j}$ associated with a Newton component $f_{j}^{q}$ of $f$ defines a quasi-ordinary power series at the root 0 . Moreover under any Newton map $\mathbf{e v}(h)$ does not increase. More precisely the set of essential variables of $\bar{h}_{q, j}$ is contained in the one of $h$ (identifying $x_{l}$ and $y_{l}$ ).

Proof. From the well-known properties of the discriminant the following identities will give the proof of the lemma.

$$
D_{z_{1}}\left(h \circ \pi_{q, j}\right)=y_{1}^{m_{1}} y_{2}^{m_{2}} \ldots y_{d}^{m_{d}}\left(D_{z}(h) \circ \pi_{q, j}\right)=y_{1}^{r_{1}} y_{2}^{r_{2}} \ldots y_{d}^{r_{d}} \varepsilon(\mathbf{y}),
$$

where $\varepsilon(0) \neq 0, m_{i} \geq 0, r_{i} \geq 0$. The conditions on $\mathbf{e v}(h)$ and the essential variables are also clear.

Let $f_{k}^{i}$ be a Newton component of $f$, hence it defines a quasi-ordinary power series. The previous lemma shows that the pull-back $f_{k}^{i} \circ \pi_{q, j}$ can be decomposed as $y_{1}^{a_{1}} y_{2}^{a_{2}} \ldots y_{d}^{a_{d}} \bar{f}_{k}^{i}$, for some power series $\bar{f}_{k}^{i} \in k\left[\left[\mathbf{y}, z_{1}\right]\right]$.

\section{Lemma 4.21.}

(1) If $\boldsymbol{\lambda}_{(i)}=\boldsymbol{\lambda}_{q}$ and $\beta_{k}^{i}=\beta_{j}^{q}$ then $\bar{f}_{k}^{i}(\mathbf{0})=0$, therefore $\bar{f}_{k}^{i}$ defines a quasi-ordinary power series. 
(2) Otherwise, $\bar{f}_{k}^{i}(\mathbf{0}) \neq 0$, and $\bar{f}_{k}^{i}$ is a unit in $k\left[\left[\mathbf{y}, z_{1}\right]\right]$.

Proof. The proof of the lemma will follow from the following description. Take one of the roots $\zeta$ of $f$ different from $z=0$. Since we are in a good system of coordinates, $\zeta$ is written as, see identity (4.4),

$$
\zeta=\alpha_{1}^{(i)} \mathbf{x}^{\boldsymbol{\lambda}_{(i)}}+\sum_{\boldsymbol{\lambda}_{(i)}<\boldsymbol{\lambda}} \alpha_{\boldsymbol{\lambda}}^{(i)} \mathbf{x}^{\boldsymbol{\lambda}}
$$

The pull-back of $\zeta$ under the Newton map $\pi_{q, j}$ is as follows.

(1) If $\boldsymbol{\lambda}_{(i)}=\boldsymbol{\lambda}_{q}$ and $\left(\alpha_{1}^{(i)}\right)^{n_{1}^{(i)}}=\beta_{j}^{q}$ then

$$
(z-\zeta) \circ \pi_{q, j}\left(\mathbf{y}, z_{1}\right)=\mathbf{y}^{\boldsymbol{\lambda}_{q}}\left(z_{1}-\sum_{\boldsymbol{\lambda}_{q}<\boldsymbol{\lambda}} \alpha_{\boldsymbol{\lambda}}^{(i)} \mathbf{y}^{\boldsymbol{\lambda}-\boldsymbol{\lambda}_{q}}\right) .
$$

(2) If $\boldsymbol{\lambda}_{(i)}=\boldsymbol{\lambda}_{q}$ but $\left(\alpha_{1}^{(i)}\right)^{n_{1}^{(i)}} \neq\left(\alpha_{j}^{q}\right)^{n_{1}^{q}}=\beta_{j}^{q}$ then

$$
(z-\zeta) \circ \pi_{q, j}\left(\mathbf{y}, z_{1}\right)=\mathbf{y}^{\boldsymbol{\lambda}_{q}}\left(z_{1}+\alpha_{j}^{q}-\alpha_{1}^{(i)}-\sum_{\boldsymbol{\lambda}_{q}<\boldsymbol{\lambda}} \alpha_{\boldsymbol{\lambda}}^{(i)} \mathbf{y}^{\boldsymbol{\lambda}-\boldsymbol{\lambda}_{q}}\right) .
$$

(3) If $\boldsymbol{\lambda}_{(i)}<\boldsymbol{\lambda}_{q}$ then

$$
(z-\zeta) \circ \pi_{q, j}\left(\mathbf{y}, z_{1}\right)=\mathbf{y}^{\boldsymbol{\lambda}_{(i)}}\left(\left(z_{1}+\alpha_{j}^{q}\right) \mathbf{y}^{\boldsymbol{\lambda}_{q}-\boldsymbol{\lambda}_{(i)}}-\alpha_{1}^{(i)}-\sum_{\boldsymbol{\lambda}_{(i)}<\boldsymbol{\lambda}} \alpha_{\boldsymbol{\lambda}}^{(i)} \mathbf{y}^{\boldsymbol{\lambda}-\boldsymbol{\lambda}_{(i)}}\right) .
$$

(4) If $\boldsymbol{\lambda}_{q}<\boldsymbol{\lambda}_{(i)}$ then

$$
(z-\zeta) \circ \pi_{q, j}\left(\mathbf{y}, z_{1}\right)=\mathbf{y}^{\boldsymbol{\lambda}_{q}}\left(z_{1}+\alpha_{j}^{q}-\alpha_{1}^{(i)} \mathbf{y}^{\boldsymbol{\lambda}_{(i)}-\boldsymbol{\lambda}_{q}}-\sum_{\boldsymbol{\lambda}_{(i)}<\boldsymbol{\lambda}} \alpha_{\boldsymbol{\lambda}}^{(i)} \mathbf{y}^{\boldsymbol{\lambda}-\boldsymbol{\lambda}_{q}}\right) .
$$

The pull-back $\bar{h}_{q, j}$ of $h$ is $\prod_{l=1}^{d} y_{l}^{N_{l}} \bar{f}_{q, j}\left(\mathbf{y}, z_{1}\right) w\left(\mathbf{y}, z_{1}\right)$ where $w(\mathbf{0}) \neq 0$. Moreover the characteristic exponents of the quasi-ordinary power series $\bar{f}_{q, j}\left(\mathbf{y}, z_{1}\right)$ are easily deduced from the characteristic exponents of $f$. In general the new quasi-ordinary power series $\bar{h}_{q, j}$ is not given in a good system of coordinates. Nevertheless the change of coordinates we need to put it in good coordinates is described in Remark 4.7.

Remark 4.22. If $\beta_{j}^{q}$ is a simple root of the polynomial $f_{\boldsymbol{\lambda}_{\kappa_{q}}}(\mathbf{1}, z)$ then $\bar{f}_{j}^{q}\left(\mathbf{0}, z_{1}\right)=$ $z_{1}+\cdots$. Using the Implicit Function Theorem the root of $\bar{f}_{j}^{q}$ is a series in $k[[\mathbf{y}]]$. As in the Puiseux Theorem $(d=1)$, all roots of $f$ can be found by using a sequence of Newton maps, (see [27, Theorem 2] for details).

If $f$ (or $h$ ) has non-degenerated Newton principal part then all roots of all $f_{\boldsymbol{\lambda}_{q}}(\mathbf{1}, z)$ are simple roots. Otherwise $f$ has degenerated principal part and following the proof of [27. Theorem 2] one can prove that by successive Newton maps and maps of type $z_{1}=z-m(\mathbf{x})$, to get again a good system of coordinates, we reach a point in which all pull-back of $f$ have non-degenerated principal part. 
Definition 4.23. We define the depth of a quasi-ordinary power series $h$, and denote it by $\operatorname{depth}(h)$, as follows. First we put $h$ in $P$-good coordinates; assume $h$ is represented using its Newton components as follows:

$$
h=x_{1}^{N_{1}} x_{2}^{N_{2}} \ldots x_{d}^{N_{d}} z^{\varepsilon} \prod_{q=1}^{r} \prod_{j=1}^{u(q)} f_{j}^{q}(\mathbf{x}, z) u(\mathbf{x}, z), \quad \text { with } u(\mathbf{0}, 0) \neq 0 .
$$

If $h=x_{1}^{N_{1}} x_{2}^{N_{2}} \ldots x_{d}^{N_{d}} z^{\varepsilon} u(\mathbf{x}, z), \varepsilon=0,1$, then we will say that $\operatorname{depth}(h):=0$. Otherwise

$$
\operatorname{depth}(h):=\max \left\{\operatorname{depth}\left(\bar{h}_{q, j}\right)\right\}+1,
$$

where the maximum is taken over all pull-backs $\bar{h}_{q, j}$ under the Newton maps associated with Newton components $f_{j}^{q}$ of $f$. If $h$ appears in good coordinates and a choice $\eta$ of good coordinates has been given after each Newton mapping, then we define in the same way $\operatorname{depth}_{\eta}(h)$.

Remark 4.24. From the above discussion $\operatorname{depth}(h) \in \mathbb{N}$ and decreases under the Newton maps. Moreover $h$ has non-degenerated Newton principal part and $h \neq$ $x_{1}^{N_{1}} x_{2}^{N_{2}} \ldots x_{d}^{N_{d}} z^{\varepsilon} u(\mathbf{x}, z)$ ( $u$ unity) if and only if $\operatorname{depth}(h)=1$. The finiteness of the invariant $\operatorname{depth}(h)$ will be used in the proof of the main result. It is easily seen that for any recursive choice $\eta$ of good coordinates, $\operatorname{depth}_{\eta}(h) \leq \operatorname{depth}(h)$. Note that the components which correspond to edges appearing after an edge with integer multi-slope need less Newton mappings to be improved.

We can keep all the informations on the Newton process in a tree, the same way, as Eisenbud and Neumann diagrams do for curves. Assume we have chosen a system of coordinates such that the Newton polygon of $h$ is a polygonal path. In our tree, each compact face will be represented by a vertex, and two vertices are connected by an edge (a vertical edge) if and only if the two faces intersect. The non compact faces are represented by arrows connected to the face they intersect. The arrow $\mathcal{F}_{0, . ., 0}$ representing $x_{i}=N_{i}, i=1, \ldots, d$ is decorated by $\left(N_{1}, \ldots, N_{d}\right)$, and the arrow $\mathcal{F}_{0}$, representing the hyperplane $z=0$ or $z=1$, is decorated by 0 or 1 . The edges are decorated as follows: let $\gamma_{q}$ be a face (represented by the vertex $v_{q}$ ) and $\boldsymbol{\lambda}_{q}=\left(\ldots, b_{l}^{q} / n_{1}^{q}, \ldots\right)$ its multislope, then the extremity of the edge attached to $v_{q}$ in the direction of $\mathcal{F}_{0}$, is decorated by $n_{1}^{q}$, and the edge attached to $v_{q}$ in the direction of $\mathcal{F}_{0, \ldots, 0}$ is decorated by $\left(b_{1}^{q}, \ldots b_{d}^{q}\right)$. Notice that the edge multideterminants are all strictly positive. Now if $f$ is non degenerated with respect to $\gamma_{q}$, we attached to $v_{q}$ as non vertical edges ending with arrows as the number of integer points on $\gamma_{q}$ minus one (recall that this is nothing but $v(q)$. If not, we consider a Newton map attached to $\gamma_{q}$ and one of its factors. We consider the pull back of $h$ under this map, and put it in coordinates such that the Newton polygon is a polygonal path. We consider the part of the tree corresponding to the new Newton polygon and we delete its arrow $\mathcal{F}_{0, \ldots, 0}$ and attached the edge to the vertex $v_{q}$, so that the edge is not vertical. Because after a finite number of steps we have something non degenerated, at the end we have a tree whose edges are attached to vertices or arrows. We can make this diagram minimal by first erasing the edges at the bottom of the tree ending 
with an arrow decorated with a 0 (we erase also this arrow) and decorated with 1 , and then the vertex on the other end of the edge if it is connected only to two other vertices. In good coordinates the diagrams are always minimal. If we are in $P$-good coordinates, there are no 1 on vertical edges. If we erase the decoration 1 on edges, and forget about vertical or horizontal edges, the diagram in good coordinates or $P$ - good coordinates are the same.

Definition 4.25. Let $h$ be a quasi-ordinary power series with a recursive choice $\eta$ of good coordinates. As usual suppose $h=x_{1}^{N_{1}} x_{2}^{N_{2}} \ldots x_{d}^{N_{d}} f(\mathbf{x}, z) u(\mathbf{x}, z)$ and assume $f$ is represented using its Newton components as: $f=z^{\varepsilon} \prod_{q=1}^{r} \prod_{j=1}^{v(q)} f_{j}^{q}, \varepsilon=0$ or 1 . Let $\omega$ be a differential form such that the pair $(h, \omega)$ verifies the support condition (2.3). According to (4.14), (4.15), (4.13) and (4.14) we consider a set $C P_{\eta}(h, \omega)$ of candidate poles for $(h, \omega)$ recursively as follows:

$$
C P_{\eta}(h, \omega):=\left\{(1,1)^{\varepsilon},\left(N_{i}, \nu_{i}\right)\right\}_{i=1}^{d} \cup \bigcup_{q=1}^{r}\left\{\left(\frac{M_{l}^{q}}{c_{l}^{q}}, \nu_{l} p_{l}^{q}+\bar{b}_{l}^{q}\right)\right\}_{l=1}^{d} \cup \bigcup C P_{\eta}\left(\bar{h}_{q, j}, \bar{\omega}_{q, j}\right),
$$

where the last union runs over all pull-back $\left(\bar{h}_{q, j}, \bar{\omega}_{q, j}\right)$ of $(h, \omega)$ under the Newton maps associated with Newton components $f_{j}^{q}$ of $f, q=1, \ldots, r$ and $j=1, \ldots, v(q)$. We will drop $\eta$ if $P$-good coordinates have been chosen.

Remark 4.26. Let $h \in k[[\mathbf{x}]][z]$ be a quasi-ordinary function with $h(\mathbf{0}, 0)=0$ such that $D_{z}(h)=x_{1}^{\alpha_{1}} x_{2}^{\alpha_{2}} \ldots x_{d}^{\alpha_{d}} \varepsilon(\mathbf{x})$, where $\varepsilon \in k[[\mathbf{x}]], \varepsilon(0) \neq 0$. After relabeling the variables $x_{i}$, one of the following conditions occurs. Either $\operatorname{ev}(h)=0$, therefore $\alpha_{i}=$ 0 for all $i=1, \ldots, d$ (which is is equivalent to $h=z^{\varepsilon}, \varepsilon=0,1$, in good coordinates) or $\alpha_{k}>0$ for all $k=1, \ldots, m \leq d$ and $\alpha_{k}=0$ for all $k \in\{m+1, \ldots, d\}$. In such a case the last $d-m$ coordinates of any characteristic exponents of $f$ are always zero. In particular compact faces of $\Gamma(h)$ are contained in the $(m+1)$-dimensional coordinate plane $x_{m+1}=\ldots=x_{d}=0$. The Newton maps never involve the last $d-m$ coordinates and therefore all compact faces of the new Newton polyhedra just only depend on the pull-back under the Newton maps of the coordinates $x_{1}, \ldots, x_{m}, z$.

Lemma 4.27. The set of candidate poles does not depend on $\eta$, i.e., it equals always the result obtained for $P$-good coordinates.

This is a consequence of the fact that the candidate poles can be read from the tree we have defined; note that the multiplicities of the differential form can be deduced from the decorated tree. This tree is the same for good coordinates and $P$-good coordinates.

Definition 4.28. We will say that two quasi-ordinary power series with recursive choices $\eta, \eta^{\prime}$ of good coordinates have the same $\left(\eta, \eta^{\prime}\right)$-characteristic exponents if their graphs are equal. We will drop the term $\left(\eta, \eta^{\prime}\right)$ if $P$-good coordinates is the common choice or if $\left(\eta, \eta^{\prime}\right)$ are clear in the context.

Next lemma states the behaviour of these graphs under the Newton mappings. 
Lemma 4.29. Let $f \in k[[\mathbf{x}]][z]$ be a quasi-ordinary power series with a recursive choice $\eta$ of good coordinates. Let $g \in k^{\prime}[[\mathbf{x}]][z]$ be other quasi-ordinary power series (maybe defined over distinct algebraically closed fields of characteristic zero $k^{\prime}$ ) with a recursive choice $\eta^{\prime}$. Then $f$ and $g$ have the same $\left(\eta, \eta^{\prime}\right)$-characteristic exponents if and only if

(1) $\Lambda_{N D}(f)=\Lambda_{N D}(g)$,

(2) there exists a bijection between the roots of the polynomials $f_{\boldsymbol{\lambda}_{q}}$ and $g_{\boldsymbol{\lambda}_{q}}$ and

(3) under this bijection the Newton component $f_{j}^{q}$ (or the root $f_{q, j}$ ) corresponds to the Newton component $g_{j}^{q}$ (or the root $g_{q, j}$ ) then the quasi-ordinary power series $\bar{f}_{q, j}$ and $\bar{g}_{q, j}$ have the same $\left(\eta, \eta^{\prime}\right)$-characteristic exponents.

It is possible to prove that the data $\eta$-characteristic exponents, that is the tree defined above, is the same as the characteristic exponents of each irreducible component of $f$ and the order of coincidence between distinct irreducible factors of $f$ (as in the case of plane curves).

Remark 4.30. Under any linear change of coordinates of type $z=\beta_{d+1} z_{1}, x_{l}=\beta_{l} y_{l}$, $l=1 \ldots, d$, with $\beta_{1}, \ldots, \beta_{d+1} \in \mathbf{G}_{m, k}$, the characteristic exponents, resp. monomials, of $f$ do not change.

\subsection{Transversal sections of a quasi-ordinary power series.}

Consider a quasi-ordinary $h \in k[[\mathbf{x}]][z]$ with $h(\mathbf{x}, z)=x_{1}^{N_{1}} x_{2}^{N_{2}} \ldots x_{d}^{N_{d}} g(\mathbf{x}, z)$, where no $x_{i}$ divides $g(\mathbf{x}, z)$ and $N_{l} \geq 0$ for any $l=1, \ldots, d$. As usual we write $h(\mathbf{x}, z)=x_{1}^{N_{1}} x_{2}^{N_{2}} \ldots x_{d}^{N_{d}} f(\mathbf{x}, z) u(\mathbf{x}, z), f(\mathbf{x}, z)$ being the quasi-ordinary Weierstrass $z$-polynomial of degree $n$ in $k[[\mathbf{x}]][z]$ whose roots are centred at $(\mathbf{0}, 0)$ and $u(\mathbf{0}, 0) \neq 0$. Assume that $h$ is given in good coordinates. Suppose that a pair $(h, \omega)$ verifying the support condition [2.3] is given, where $\omega=\prod_{j=1}^{d} x_{j}^{\nu_{j}-1} d x_{1} \wedge \ldots \wedge d x_{d} \wedge d z, \nu_{j} \geq 1$.

Fix $i \in\{1, \ldots, d\}$, and suppose that $d \geq 2$. Let $k_{i}$ denote an algebraic closure of the fraction field of the formal power series ring $k\left[\left[x_{i}\right]\right]$. The $i$-th transversal section of $h$ will be a finitely many disjoint union of $k_{i}$-quasi-ordinary formal power series obtained from $h$ and their corresponding differential forms verifying the support condition 2.3. Let $\mathbf{0}_{i}$ denote the $d$-tuple which has all coordinates but the $i$-th one, which is $x_{i}$, equal 0 . Consider the polynomial $f\left(\mathbf{0}_{i}, z\right)$ over $k_{i}$ of degree $n$ :

$$
f\left(\mathbf{0}_{i}, z\right)=z^{n}+\text { lower degree terms }=\prod_{m=1}^{v_{i}}\left(z-\alpha_{m}\right)^{\delta_{m}} \in k_{i}[z],
$$

$\alpha_{m} \in k_{i}$ and $n=\sum \delta_{m}$. Since the discriminant specializes, for each root $\alpha_{m}$ of $f\left(\mathbf{0}_{i}, z\right)=0$ in $k_{i}[z]$, Jung-Abhyankar Theorem shows that there exists a $k_{i}$-quasiordinary formal power series $h_{i}^{\alpha_{m}} \in k_{i}\left[\left[\widehat{\mathbf{x}}_{i}\right]\right][z]$ centred at $\left(\widehat{\mathbf{0}}_{i}, \alpha_{m}\right), \widehat{\mathbf{x}}_{i}$ being all variables but $x_{i}$.

Definition 4.31. The $i$-transversal section of $h$ consists in the set $\left\{h_{i}^{\alpha_{m}}: m=\right.$ $\left.1, \ldots, v_{i}\right\}$ of $k_{i}$-quasi-ordinary formal power series, $h_{i}^{\alpha_{m}}$ centred at $\left(\widehat{\mathbf{0}}_{i}, \alpha_{m}\right)$. It is clear that $\mathbf{e v}\left(h_{i}^{\alpha_{m}}\right)<d$. If the root $\alpha_{m} \neq 0$ then we need a translation of type 
$z_{1}=z-\alpha_{m}$ to describe the corresponding $h_{i}^{\alpha_{m}}$. On the other hand, if $x_{i}$ is not an essential variable for $h$ then the only root is $\alpha=0$.

Since we are also interested in keeping the differential form, the $i$-transversal section of a pair $(h, \omega)$ consists in the set of finitely many pairs $\left(h_{i}^{\alpha_{m}}, \omega_{i}\right)$ where

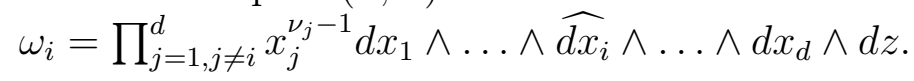

Example 4.32. Consider $f\left(x_{1}, x_{2}, z\right)=\left(\left(z^{2}-x_{1}^{3}\right)^{2}+x_{1}^{7}\right)^{3}+x_{1}^{25} x_{2}^{3}$. For $i=1$, the 1 -transversal sections are the $k_{1}$-quasi-ordinary power series centred at the roots of $\left(\left(z^{2}-x_{1}^{3}\right)^{2}+x_{1}^{7}\right)^{3}=0$ in $k_{1}$. In fact these roots can be found using the Newton-Puiseux algorithm in $k_{1}$ for the previous polynomial. For $i=2$, the 2-transversal section is the $k_{2}$-quasi-ordinary power series $f_{2}^{0}=\left(\left(z^{2}-x_{1}^{3}\right)^{2}+x_{1}^{7}\right)^{3}+\left(x_{2}^{3}\right) x_{1}^{25} \in k_{2}\left[x_{1}, z\right]$ centred at the root $\alpha=0$. In fact it is clear that the Newton polyhedron of $f_{2}^{0}$ is the projection over the plane $x_{2}=0$ of $\Gamma(f)$.

A description of the components $h_{i}^{\alpha}$ of the $i$-transversal section is as follows. Assume that

$$
f(\mathbf{x}, z)=\prod_{j=1}^{n}\left(z-\zeta_{j}\left(x_{1}^{1 / m}, \ldots, x_{d}^{1 / m}\right)\right)
$$

with $\zeta_{j}(\mathbf{0})=0$. If $\zeta_{j} \neq 0$, since $f$ is given in good coordinates, then

$$
\zeta_{j}(\mathbf{x})=\alpha_{1}^{(j)} \mathbf{x}^{\boldsymbol{\lambda}_{(j)}}+\sum_{\boldsymbol{\lambda}_{(j)}<\boldsymbol{\lambda}} \alpha_{\boldsymbol{\lambda}}^{(j)} \mathbf{x}^{\boldsymbol{\lambda}},\left(\alpha_{1}^{(j)} \neq 0\right) .
$$

The characteristic exponents of type $\left(\mathbf{0}, \frac{b_{i}}{n_{1}}, \mathbf{0}\right) \in \mathbb{Q}^{d}$, correspond to 1-dimensional faces in $C F(f)$ contained in the $\left(x_{i}, z\right)$-plane. We write in a unique way $\zeta_{j}(\mathbf{x})$ as sum of two formal power series $p_{j}\left(x_{i}^{1 / m}\right)+t_{j}(\mathbf{x})$ such that $p_{j}\left(x_{i}^{1 / m}\right) \in k\left[\left[x_{i}^{1 / m}\right]\right]$ is the sum of monomials of $\zeta_{j}$ depending only on $x_{i}$ and $t_{j}=\zeta_{j}-p_{j}\left(x_{i}\right)$. It is clear that $t_{j}\left(\mathbf{0}_{i}\right)=0$.

If $\alpha \in k_{i}$ is a root of $f\left(\mathbf{0}_{i}, z\right)=0, h_{i}^{\alpha}\left(\widehat{\mathbf{x}}_{i}, z\right)$ is

$$
h_{i}^{\alpha}=u(\mathbf{x}, z) \prod_{l=1}^{d} x_{l}^{N_{l}} \prod_{j: p_{j}\left(x_{i}^{1 / m}\right)=\alpha}\left(z-\left(\alpha+t_{j}\left(x_{1}^{1 / m}, \ldots, \widehat{x}_{i}, \ldots, x_{d}^{1 / m}\right)\right) \in k_{i}\left[\left[\widehat{\mathbf{x}}_{i}\right]\right][z],\right.
$$

where $t_{j}$ is seen as an element in $k_{i}\left[\left[x_{1}^{1 / m}, \ldots, \widehat{x}_{i}, \ldots, x_{d}^{1 / m}\right]\right]$. The last product gives a decomposition of $h_{i}^{\alpha}$ in its irreducible roots too, because each factor has $z$-degree one. To work with $h_{i}^{\alpha}$ we first do, if necessary, the translation $z_{1}=z-\alpha, \alpha \in k_{i}$. Such a translation is a composition of some Newton maps of $h$ associated with their corresponding Newton components (all of them of type $\left.\left(\mathbf{0}, a_{i} / w_{i}, \mathbf{0}\right)\right)$ and some change of variables for having good coordinates. The Newton map $\pi_{1}$ associated with a Newton component of $h$ of type $\left(\mathbf{0}, b_{i} / n_{1}, \mathbf{0}\right)$ is given by $x_{i}=y_{i}^{p_{i}}, z=\left(z_{1}+\alpha\right) y_{i}^{\bar{b}_{i}}$ and $x_{j}=y_{j}$, if $j \neq i$. After $\pi_{1}$, we need a translation of type $z_{1}=\tilde{z}-\phi(\mathbf{x})$ to get good coordinates. This translation does not change the position of the $i$-th variable. If in order to get $\alpha$ maybe we will need more Newton maps of type $\left(\mathbf{0}, a_{i} / w_{i}, \mathbf{0}\right)$ 
and we finish when no more characteristic exponent of the mentioned type appear to get $\alpha$. Therefore the composition of these Newton maps and their corresponding translations, which we denote by $\pi$, is the same as the Newton-Puiseux algorithm to find the root $\alpha$ of $f\left(\mathbf{0}_{i}, z\right)$ in $k_{i}[z]$. The map $\pi$ does nothing on the set of variables different from $i$-th one. Let $\tilde{f}$ (resp. $\tilde{h}$ ) denote the pull-back of $f$ (resp. $h$ ) under the map $\pi$. Up to a factor $x_{i}^{k / m}, \tilde{f}(\mathbf{x}, z)=\prod_{j=1}^{n}\left(z-t_{j}\left(x_{1}^{1 / m}, \ldots, x_{d}^{1 / m}\right)\right.$ and

$$
h_{i}^{\alpha}\left(\widehat{\mathbf{x}}_{i}, z\right)=\tilde{u} \prod_{l=1}^{d} x_{l}^{N_{l}} u(\mathbf{x}, z) \prod_{j: p_{j}\left(x_{i}^{1 / m}\right)=\alpha}\left(z-t_{j}\left(x_{1}^{1 / m}, \ldots, \widehat{x}_{i}, \ldots, x_{d}^{1 / m}\right)\right) .
$$

Moreover, $\omega_{i}$ and the pull-back $\pi^{*} \omega$ are the same, up to a constant, as differential forms over $k_{i}$.

Once this translation is done, the Newton polyhedron $\Gamma_{i}\left(h_{i}^{\alpha}\right)$ of $h_{i}^{\alpha}$ as $k_{i}$-quasiordinary power series is the projection over the hyperplane $x_{i}=0$ of the Newton polyhedron of the pull-back $\tilde{f}$; this is clear for $\alpha=0$ and for the other case is similar. In particular $\Gamma_{i}\left(h_{i}^{\alpha}\right)$ is a monotone polygonal path. In fact, the $k_{i}$-quasi-ordinary power series $h_{i}^{\alpha}$ is in good coordinates because the condition not to be a power of a linear form is generic.

Remark 4.33. Note that if $h$ is given in $P$-good coordinates, we cannot deduce that $h_{i}^{\alpha}$ is also in $P$-good coordinates. This is the main reason for introducing the more general concept of good coordinates.

Example 4.34. (1) Consider $f\left(x_{1}, x_{2}, z\right)=\left(z^{2}-x_{1}^{2} x_{2}^{5}\right)\left(z^{2}-x_{1}^{2} x_{2}^{7}\right)$. In principle $\Gamma(f)$ has two edges which are projected over just only one edge if we project over $x_{2}=0$. The 2-transversal section has at least two different components with the same characteristic exponent. (2) $f\left(x_{1}, x_{2}, z\right):=\left(z^{2}-x_{1}^{2} x_{2}^{5}\right)^{2}+x_{1}^{4} x_{2}^{17}$. Seeing this as a 2-transversal section one has $f_{2}^{0}=z^{4}-\left(2 x_{2}^{5}\right) x_{1}^{4} z+\left(x_{2}^{10}+x_{2}^{17}\right) x_{1}^{4}$. In this case some more monomials of $x_{1}^{4}$ have appeared. These monomials are hidden in $N D(f)$.

To sum up:

Proposition 4.35. With the above notations,

(1) The formal power series $h_{i}^{\alpha_{m}} \in k_{i}\left[\left[\widehat{\mathbf{x}}_{i}\right]\right][z]$ centred at $\left(\widehat{\mathbf{0}}_{i}, \alpha_{m}\right)$, defines a quasiordinary power series with $\mathbf{e v}\left(h_{i}^{\alpha_{m}}\right)<d$.

(2) The Newton process for $h$ induces Newton process for $h_{i}^{\alpha_{m}}$. Each step of the Newton process for $h$ is necessary for at least one $h_{i}^{\alpha_{m}}, i \in\{1, \ldots, d\}$, and $m \in\left\{1, \ldots, v_{i}\right\}$.

(3) Fix $i \in\{1, \ldots, d\}$ and $m \in\left\{1, \ldots, v_{i}\right\}$. Then

i) If $\alpha_{m}=0$ then the quasi-ordinary power series $h_{i}^{0}$ is in good coordinates.

ii) At each step of the Newton process for $h, \Gamma\left(h_{i}^{0}\right)$ is the projection of $\Gamma(h)$ on the hyperplane $x_{i}=0$.

iii) If $\alpha_{m} \neq 0$, then some steps of the Newton process for $h$ firstly provide the translation $z=z_{1}-\alpha$ we need to put $\alpha$ at zero. After that we apply $i$ ) and ii). 
iv) Every pair $\left(h_{i}^{\alpha_{m}}, \omega_{i}\right)$ verifies the support condition (2.3).

(4) In fact

$$
C P(h, \omega) \subset \bigcup_{i=1}^{d} \bigcup_{m=1}^{v} C P\left(h_{i}^{\alpha_{m}}, \omega_{i}\right),
$$

Recall that the second union runs over all roots of $f\left(\mathbf{0}_{i}, z\right)=0$ in $k_{i}[z]$.

The only fact in the proposition that is not yet proved is the equality (4.19). The set $C P(h, \omega)$ of candidate poles for $(h, \omega)$ can be written as $C P(h, \omega)=C P_{1}(h, \omega) \cup$ $\bigcup C P\left(\bar{h}_{q, j}, \bar{\omega}_{q, j}\right)$, see definition 4.25, where the set $C P_{1}(h, \omega)$ is completely described from $N D(h)$ and $\omega$. Since this monotone polygonal path is determined by (and determines) its projections over all hyperplanes $\left\{x_{i}=0\right\}, i=1, \ldots, d$, then

$C P_{1}(h, \omega)=\bigcup_{i=1}^{d} C P_{1}\left(h_{i}^{0}, w_{i}\right)$. In the previous identity we forget the roots which are not centred at zero because some Newton maps are needed to find them.

In general, (4.19) follows from the fact that candidate poles are determined by the characteristic monomials of the quasi-ordinary power series. The characteristic monomials are determined by difference of its roots. In particular for each of the $i$-transversal section the difference of two of its roots in $k_{i}\left[\left[x_{1}^{1 / m}, \ldots, \widehat{x}_{i}, \ldots, x_{d}^{1 / m}\right]\right]$ can be computed as the specialization of the difference of the corresponding roots in $k\left[\left[x_{1}^{1 / m}, \ldots, x_{i}^{1 / m}, \ldots, x_{d}^{1 / m}\right]\right]$.

In fact we can say more. Fix a characteristic exponent of $f$ and a Newton component with such a characteristic exponent. Fix $i \in\{1, \ldots, d\}$. If the characteristic exponent is $\left(\mathbf{0}, b_{i} / n_{1}, \mathbf{0}\right)$ then we have already described what is going on with the Newton map $\pi$ associated with the Newton component. Otherwise, reading the characteristic exponents and the roots from equations (4.17) and (4.18), the characteristic exponents of the $i$-transversal section of $f \circ \pi$ are the same as those one for the pull-back under the corresponding Newton map for the $i$-transversal section. In particular at each step the Newton polyhedron of the $i$-transversal section is the projection of the Newton polyhedron of $h$.

The fact that we get the same differential form, up to a constant factor, in $x_{i}$ is clear because this is a general fact no related with quasi-ordinary formal power series.

\section{Denef-Loeser motivic zeta function under the NeWton maps}

We keep the field $k$ being algebraically closed and denote an algebraic closure of the quotient field $k((t))$ of the domain $R:=k[[t]]$ by $K$. The goal is to compute zeta functions for a $k$-quasi-ordinary power series using induction on its depth. In the last section we have computed the $A$-part. In order to compute the $B$-part we must take into account that a quasi-ordinary power series may be degenerated with respect to its Newton polyhedron. The main idea is to use Newton maps to measure some sets of arcs in terms of the quasi-ordinary power series issued after these Newton maps. The problem is the existence of some arcs which cannot be lifted under such maps. We need to combine Newton maps and $k[t]$-morphisms to solve this problem. 
Therefore we introduce technical objects, called $\mathcal{W}$ and $\widetilde{\mathcal{W}}$-quasi-ordinary series in order to have families of power series closed under Newton maps to deal with. We will compute the Denef-Loeser motivic zeta functions for these series following the ideas of subsections 3.2 and 4.3 the computation of the $A$-part is similar to the one in 4.3 but there are some small differences and we will compute inductively the whole zeta function.

Definition 5.1. Let $h^{t}(\mathbf{x}, z) \in R\left[\left[x_{1}, \ldots, x_{d}\right]\right][z]$ be a formal power series such that $h^{t}=t^{\theta} x_{1}^{N_{1}} x_{2}^{N_{2}} \ldots x_{d}^{N_{d}} f^{t}(\mathbf{x}, z) u^{t}(\mathbf{x}, z)$ where $x_{j}$ does not divide $f^{t}(\mathbf{x}, z) \forall j=1, \ldots, d$, $t$ does not divide $f^{t}(\mathbf{x}, z) u^{t}(\mathbf{x}, z), \theta \in \mathbb{N}$ and $u^{t}(\mathbf{0}, 0) \neq 0$. We say that $h^{t}$ is $\mathcal{W}$ quasi-ordinary if its $z$-discriminant is

$$
D_{z}\left(f^{t}\right)=t^{\beta} x_{1}^{\alpha_{1}} x_{2}^{\alpha_{2}} \ldots x_{d}^{\alpha_{d}} \varepsilon(t, \mathbf{x}),
$$

where $\varepsilon(0, \mathbf{0}) \neq 0$; therefore if we consider $t$ as a variable, $f^{t} \in k[[t, \mathbf{x}]][z]$ is a $k$ quasi-ordinary power series. Let $J \subset\{1 \ldots, d\}$ and let $J^{\prime}$ be its complement; we say that $h^{t}$ is $J$-bounded if its roots $\zeta^{t}$ verify

$$
\zeta^{t} \in k\left[\mathbf{x}_{J}^{1 / m}\right]\left[\left[\mathbf{x}_{J^{\prime}}^{1 / m}, t\right]\right], \text { for some } \mathrm{m} \text {. }
$$

Remark 5.2. The main property of a $J$-bounded $\mathcal{W}$-quasi-ordinary series $h^{t}$ is that $\forall \mathbf{y}_{J}^{0} \in \mathbb{C}^{d}$ such that the entries in $J^{\prime}$ are zero the series $h^{t}\left(\mathbf{x}+\mathbf{y}_{J}^{0}, z\right)$ is a well-defined $\mathcal{W}$-quasi-ordinary series.

Let $f(\overline{\mathbf{x}}, \bar{z}) \in k\left[\bar{x}_{1}, \ldots, \bar{x}_{d}\right][\bar{z}]$ be a quasi-ordinary polynomial whose roots only contain the (finite set of) characteristic monomials of $f^{t}$ as $K$-quasi-ordinary power series in a new set of variables $\bar{x}_{1}, \ldots, \bar{x}_{d}, \bar{z}$. Let $h:=\bar{x}_{1}^{N_{1}} \ldots \bar{x}_{d}^{N_{d}} f(\overline{\mathbf{x}}, \bar{z})$. In particular the $K$-quasi-ordinary power series $h^{t}$ and the $k$-quasi-ordinary power series $h$ have the same characteristic exponents and the same characteristic monomials.

If $f^{t}(\mathbf{x}, z) \in k[[t]][[\mathbf{x}]][z]$ is not in good coordinates, by Remark 4.7] the change of coordinates to put $f^{t}(\mathbf{x}, z)$ in good coordinates is an automorphism of $k[[t]][[\mathbf{x}]][z]$ of type $z \mapsto z+m^{t}(\mathbf{x}), m \in k[[t]][[\mathbf{x}]]$. In particular the $t$-variable does not change and condition (5.1) on the discriminant is preserved. It is easily seen that if $h^{t}$ is $J$-bounded then condition (5.2) is also preserved because $m^{t}(\mathbf{x})$ comes from some monomials of the roots of $h^{t}$. Then we assume $f^{t}$ and $f(\overline{\mathbf{x}}, \bar{z})$ are in good coordinates.

Consider differential forms $\omega=\prod_{j=1}^{d} x_{j}^{\nu_{j}-1} d \mathbf{x} \wedge d z$ and $\bar{\omega}=\prod_{j=1}^{d} \bar{x}_{j}^{\nu_{j}-1} d \overline{\mathbf{x}} \wedge d \bar{z}$, with $\nu_{j} \geq 1$. From now on we assume that $(h, \bar{\omega})$ verifies the support condition 2.3, Let $X:=\mathbb{A}_{k}^{d+1}$ and set

$$
V_{n, m}^{t}:=\left\{\boldsymbol{\varphi} \in \mathcal{L}_{0}(X): \operatorname{ord}\left(h^{t} \circ \varphi\right)=n+\theta, \operatorname{ord}(\omega \circ \varphi)=m\right\} ;
$$

define $V_{n, m}$ in the same way for $h$ (forgetting $\theta$ ). The sets $V_{n, m}^{t}$ are measurable because of the support condition. We define the power series

$$
\begin{aligned}
Z_{D L}^{\mathcal{W}}\left(h^{t}, \omega, T\right) & :=T^{\theta} \sum_{n \in \mathbb{N}}\left(\sum_{m \in \mathbb{N}} \mathbb{L}^{-m} \mu_{X}\left(V_{n, m}^{t}\right)\right) T^{n} \in \widehat{\mathcal{M}}_{k}[[T]], \\
Z_{\text {top }, 0}^{\mathcal{W}}\left(h^{t}, \omega, s\right) & :=\chi_{\mathrm{top}}\left(Z_{D L}^{\mathcal{W}}\left(h^{t}, \omega, \mathbb{L}^{-s}\right)\right) .
\end{aligned}
$$


Recall that

$$
\begin{array}{r}
Z_{D L}(h, \bar{\omega}, T)=\sum_{n \in \mathbb{N}}\left(\sum_{m \in \mathbb{N}} \mathbb{L}^{-m} \mu_{X}\left(V_{n, m}\right)\right) T^{n} \\
Z_{\text {top }, 0}(h, \bar{\omega}, s)=\chi_{\text {top }}\left(Z_{D L}\left(h, \bar{\omega}, \mathbb{L}^{-s}\right)\right) .
\end{array}
$$

We know from the results of Denef and Loeser that $Z_{D L}(h, \bar{\omega}, T)$ is a rational function. The last part of the section is devoted to prove the rationality of $Z_{D L}^{\mathcal{W}}\left(h^{t}, \omega, T\right)$ and to provide a small set of candidate poles for such functions, see definition 4.25. Recent interesting results of J. Sebag [30] prove the rationality of this power series.

Theorem 5.3. If $h^{t}$ is $\mathcal{W}$-quasi-ordinary then the zeta function

$$
Z_{D L}^{\mathcal{W}}\left(h^{t}, \omega, T\right) \in \mathbb{Z}\left[\mathbb{L}, \mathbb{L}^{-1},\left(1-\mathbb{L}^{-\nu} T^{N}\right)^{-1}\right][T]
$$

where $(N, \nu) \in C P(h, \bar{\omega})$. Moreover, $Z_{\mathrm{top}, 0}^{\mathcal{W}}\left(h^{t}, \omega, s\right)$ exists.

We need to define a subfamily of $\mathcal{W}$-quasi-ordinary series in order to obtain more information on the local topological zeta function.

Definition 5.4. A symbol $\widetilde{\mathcal{W}}:=\left(p ; g_{1}, \ldots, g_{d}\right)$, where $p \in \mathbb{P}, g_{i} \in \mathbb{N}, i=1, \ldots, d$, is called a weight. The semigroup $\Gamma_{\widetilde{\mathcal{W}}} \subset \mathbb{N}^{d}$ associated with $\widetilde{\mathcal{W}}$ is the additive set of $\boldsymbol{\alpha} \in \mathbb{N}^{d}$ such that

$$
w_{\widetilde{\mathcal{W}}}(\boldsymbol{\alpha}):=\sum_{i=1}^{d} \frac{\alpha_{i} g_{i}}{p} \in \mathbb{N} .
$$

Definition 5.5. Let $h^{t}(\mathbf{x}, z) \in R\left[\left[x_{1}, \ldots, x_{d}\right]\right][z]$ be a formal power series such that $h^{t}=t^{\theta} x_{1}^{N_{1}} x_{2}^{N_{2}} \ldots x_{d}^{N_{d}} f^{t}(\mathbf{x}, z) u^{t}(\mathbf{x}, z)$ with $x_{j}$ does not divide $f^{t}(\mathbf{x}, z) \forall j=1, \ldots, d$, $u^{t}(\mathbf{0}, 0) \neq 0, t$ does not divide $f^{t}(\mathbf{x}, z) u^{t}(\mathbf{x}, z)$ and $\theta \in \mathbb{N}$. We call it $\widetilde{\mathcal{W}}$-quasiordinary if it is $\mathcal{W}$-quasi-ordinary and all monomials in $h^{t}$ are of type $t^{w} \widetilde{\mathcal{W}}^{(\boldsymbol{\alpha})} \mathbf{x}^{\boldsymbol{\alpha}} z^{n}$, where $\boldsymbol{\alpha} \in \Gamma_{\widetilde{\mathcal{W}}}$ (and some non-zero coefficient in $k$ ).

Remark 5.6. If $h^{t}$ is $\widetilde{\mathcal{W}}$-quasi-ordinary then the power series $h^{1}:=h_{\mid t=1}^{t}$ is $k$-quasiordinary and have the same characteristic exponents as $h^{t}$. If $h^{t}$ is $\widetilde{\mathcal{W}}$-quasi-ordinary, it is true also for $f^{t}$ because the $d$-tuple $\left(N_{1}, \ldots, N_{d}\right) \in \Gamma_{\widetilde{\mathcal{W}}}$ and $\theta=w_{\widetilde{\mathcal{W}}}\left(N_{1}, \ldots, N_{d}\right)$. Moreover, if we put $h^{t}$ in good coordinates, the new series is also $\widetilde{\mathcal{W}}$-quasi-ordinary.

Remark 5.7. Let $h^{t}$ be a $\widetilde{\mathcal{W}}$-quasi-ordinary power series, $\widetilde{\mathcal{W}}:=\left(p ; g_{1}, \ldots, g_{d}\right)$. Then, if $J:=\left\{j \mid 1 \leq j \leq d, g_{j} \neq 0\right\}$ then $h^{t}$ is $J$-bounded.

Definition 5.8. Let $h^{t}$ be a $\widetilde{\mathcal{W}}$-quasi-ordinary power series. We say that $h^{t}$ is Newton compatible if the number of irreducible factors (different from $t$ ) of $h_{\mid N D\left(h^{t}\right)}^{t}$ in $R$ equals the number of irreducible factors of $h_{\mid N D\left(h^{1}\right)}^{1}$ in $k$. For instance $z^{2}-t x^{2} y^{2}$ is not Newton compatible and it is $\widetilde{\mathcal{W}}$-quasi-ordinary for some adequate weight. 
Theorem 5.9. If $h^{t}$ is $\widetilde{\mathcal{W}}$-quasi-ordinary and Newton compatible then

$$
Z_{\mathrm{top}, 0}^{\mathcal{W}}\left(h^{t}, \omega, s\right)=Z_{\mathrm{top}, 0}(h, \bar{\omega}, s) .
$$

From now on we suppose that $h^{t}$ is $\mathcal{W}$-quasi-ordinary; we will indicate explicitly when we consider it as $\widetilde{\mathcal{W}}$-quasi-ordinary. The rest of this section is devoted to the proof of both theorems. We will focus on the proof of Theorem 5.3 and we will point out the special arguments required for the proof of Theorem [5.9. Since the results do not depend on the factor $T^{\theta}$, we can omit it. From now on we assume that $h^{t}$ (or $f^{t}$ ) is given in good coordinates. The proofs are given by induction on the depth.

Step 1. $\operatorname{depth}(h)=\operatorname{depth}\left(h^{t}\right)=0$.

Then $N D(h)$ has only one compact face which is 0-dimensional,

$$
Z_{D L}^{\mathcal{W}}\left(h^{t}, \omega, T\right)=\mathbb{L}^{-(d+1)}(\mathbb{L}-1)^{d+1} \frac{\mathbb{L}^{-\left(1+\sum \nu_{j}\right)} T^{1+\sum N_{j}}}{1-\mathbb{L}^{-1} T} \prod_{j=1}^{d} \frac{1}{1-\mathbb{L}^{-\nu_{j}} T^{N_{j}}},
$$

and $Z_{D L}^{\mathcal{W}}\left(h^{t}, \omega, T\right)=Z_{D L}(h, \bar{\omega}, T)$. Then $Z_{\mathrm{top}, 0}^{\mathcal{W}}\left(h^{t}, \omega, s\right)=Z_{\mathrm{top}, 0}(h, \bar{\omega}, s)$.

Step 2. Assume that $\operatorname{depth}(h)>0$.

We keep the notation of $\oint_{3}$. Let $S$ be defined by $x_{1} \cdots x_{d} z=0$, ( or $\left.\bar{x}_{1} \cdots \bar{x}_{d} \bar{z}=0\right)$; we consider arcs in $\boldsymbol{\varphi} \in \mathcal{L}_{0}(X) \backslash \mathcal{L}_{0}(S)$ and define $\mathbf{k}(\boldsymbol{\varphi})$ and $\mathbf{a}(\boldsymbol{\varphi})$ as in $\$ 3$,

The following easy remark will be a key point to understand the dual decomposition associated with $f^{t}(\mathbf{x}, z) \in R[[\mathbf{x}]][z]$. Each $\varphi \in \mathcal{L}_{0}(X)$ univocally defines an arc $\boldsymbol{\psi}:=(t, \boldsymbol{\varphi}) \in \mathcal{L}_{0}\left(\mathbb{A}_{k}^{1} \times X\right)$ and $\operatorname{ord}_{t}\left(f^{t} \circ \boldsymbol{\varphi}\right)=\operatorname{ord}_{t}(g \circ \boldsymbol{\psi})$, where $g(y, \mathbf{x}, z) \in k[[y, \mathbf{x}]][z]$ is the same function as $f^{t}$ but $t$ is substituted by a new variable $y$.

Step 3. Newton polyhedron of $h \in k[\overline{\mathbf{x}}, \bar{z}]$.

This Newton polyhedron $\Gamma(h)$ and its dual decomposition have been described in paragraph 4.3. Denote by $\bar{\gamma}_{1}, \ldots, \bar{\gamma}_{r}$ its 1-dimensional faces with corresponding vertices $\bar{\tau}_{0}, \bar{\tau}_{1}, \ldots, \bar{\tau}_{r}$. From now on we assume that $\bar{\tau}_{r}$ is the $\bar{z}$-highest vertex. Thus we can write

$$
f_{N D(f)}=z^{\varepsilon} \prod_{q=1}^{r} \prod_{\bar{j}=1}^{v(q)}\left(f_{q, \bar{j}}\right)^{m_{q, \bar{j}}} \text { where } f_{q, \bar{j}}:=\bar{z}^{n_{1}^{q}}-\bar{\beta}_{\bar{j}}^{q} \bar{x}_{1}^{b_{1}^{q}} \ldots \bar{x}_{d}^{b_{d}^{q}},
$$

with $\varepsilon=0$ or 1 depending if $I=I^{\prime}$ or not. Recall that in the dual space, the reduced integer equation of the hyperplane $l^{q}$ is $\eta_{q}\left(v_{1}, \ldots, v_{d}, v_{d+1}\right)=0$, see equation (4.5). The intersection of these hyperplanes with $\mathbb{R}_{>0}^{d+1}$ are the $d$-dimensional cones $\Delta_{\bar{\gamma}_{q}}$. They determine the $(d+1)$-dimensional cones $\Delta_{\bar{\tau}_{q}}, q=0,1, \ldots, r$. The cones associated with compact faces of $\Gamma(h)$ (or $\Gamma(f))$ give a partition of $\mathbb{R}_{>0}^{d+1}$ in the disjoint union $\cup \Delta_{\bar{\tau}}$.

If $\varphi \in \mathcal{L}_{0}(X) \backslash \mathcal{L}_{0}(S)$ and $\mathbf{k}:=\mathbf{k}(\varphi)$, the order of the differential form $\bar{\omega}$ is $m(\mathbf{k}):=\sigma_{\bar{\omega}}(\mathbf{k})-\mathbf{k}$. If $\mathbf{k} \in \mathbb{P}^{d+1}$ is fixed, the semialgebraic subset $V_{n, m}^{\mathbf{k}}$ is naturally 
defined. It is empty unless $m=m(\mathbf{k})$. Then for each compact face $\bar{\tau}$ define

$$
Z_{D L}^{\bar{\tau}}(h, \bar{\omega}, T):=\sum_{\mathbf{k} \in \Delta_{\bar{\tau}} \cap \mathbb{P}^{d+1}} Z_{D L}^{\mathbf{k}}(T) .
$$

Step 4. Description of the Newton polyhedron $\Gamma^{t}\left(f^{t}\right)$ of $f^{t} \in k[[t, \mathbf{x}]][z]$.

The discriminant condition (5.1) implies that $f^{t}$ is a $k$-quasi-ordinary power series in good coordinates in $(d+2)$ variables. Then its Newton polyhedron $\Gamma^{t}\left(f^{t}\right)$ has only 0 and 1-dimensional compact faces, say $m$ edges $\tilde{\gamma}_{1}, \ldots, \tilde{\gamma}_{m}$ and the $m+1$ corresponding vertices. We can write

$$
f_{N D\left(f^{t}\right)}^{t}=z^{\varepsilon} \prod_{p=1}^{m} \prod_{s=1}^{v(p)}\left(z^{\tilde{n}_{1}^{p}}-\beta_{s}^{p} \tilde{t}_{0}^{p} x_{1}^{\tilde{b}_{1}^{p}} \ldots x_{d}^{\tilde{b}_{d}^{p}}\right)^{m_{p, s}},
$$

where $\operatorname{gcd}\left(\tilde{n}_{1}^{p}, \tilde{b}_{0}^{p}, \tilde{b}_{1}^{p}, \ldots, \tilde{b}_{d}^{p}\right)=1$ and $\varepsilon=0$ or 1 depending if $I=I^{\prime}$ or not.

The characteristic exponents associated with $\Gamma^{t}\left(f^{t}\right)$ are of type $\left(\frac{\tilde{b}_{0}^{p}}{\tilde{n}_{1}^{p}}, \boldsymbol{\lambda}_{p}^{t}\right) \in \mathbb{Q}^{d+1}$ where $\tilde{n}_{1}^{p} \boldsymbol{\lambda}_{p}^{t}=\left(\tilde{b}_{1}^{p}, \ldots, \tilde{b}_{d}^{p}\right) \in \mathbb{N}^{d}$. We need also to define $n_{1}^{p}, b_{1}^{p}, \ldots, b_{d}^{p}$ such that $\operatorname{gcd}\left(n_{1}^{p}, b_{1}^{p}, \ldots, b_{d}^{p}\right)=1$ and $n_{1}^{p} \boldsymbol{\lambda}_{p}^{t}=\left(b_{1}^{p}, \ldots, b_{d}^{p}\right)$.

Given $\boldsymbol{\lambda}_{q}$ from a compact face $\bar{\gamma}_{q}$ of $\Gamma(h), q=1, \ldots, r$, we collect the set $S_{q}$ of rationals $\frac{b}{n}$ such that there exists a compact face $\tilde{\gamma}_{p}$ of $\Gamma^{t}\left(h^{t}\right)$ whose associated characteristic exponent is $\left(\frac{b}{n}, \boldsymbol{\lambda}_{q}\right)$; all characteristic exponents of $h^{t}$ are obtained in this way.

Let $u(q)$ be the cardinality of $S_{q}$; we denote by $\frac{\alpha_{q, j}}{n_{q, j}}, j=1, \ldots, u(q)$, the elements of $S_{q}$, where $\alpha_{q, j} \in \mathbb{N}$ and $n_{1}^{q, j}=\tilde{n}_{1}^{p}$ for the corresponding $p \in\{1, \ldots, m\}$. We denote also $s_{1}^{q, j}:=\frac{n_{1}^{q, j}}{n_{1}^{q}} \in \mathbb{N}$. Then identity (5.3) can be rewritten as follows

$$
f_{N D\left(f^{t}\right)}^{t}=z^{\varepsilon} \prod_{q=1}^{r} \prod_{j=1}^{u(q)} \prod_{w=1}^{w(q, j)}\left(\left(z^{n_{1}^{q}}\right)^{s_{1}^{q, j}}-\beta_{w} t^{\alpha_{q, j}}\left(x_{1}^{b_{1}^{q}} \ldots x_{d}^{b_{d}^{q}}\right)^{s_{1}^{q, j}}\right)^{m_{q, j, w}},
$$

where $v(q)=\sum_{j=1}^{u(q)} s_{1}^{q, j} w(q, j)$ since $f^{t}$ and $f$ have by hypothesis the same characteristic exponents.

Remark 5.10. If $h^{t}$ is $\widetilde{\mathcal{W}}$-quasi-ordinary and Newton compatible then $r=m$, it means that for each $q=1, \ldots, r$ we have $\# S_{q}=1$ and $S_{q} \in \mathbb{N}$. In this case $s_{1}^{q, j}=1$, $\tilde{b}_{j}^{q}=b_{j}^{q}$.

In the dual space $\left(\mathbb{R}^{d+2}\right)^{*}$, with coordinates $\left(v_{0}, v_{1}, \ldots, v_{d}, v_{d+1}\right)$, the dual decomposition induced by $\Gamma^{t}\left(h^{t}\right)$ is given by dual cones $\Delta_{\tilde{\gamma}_{p}}$ associated with the hyperplanes, $\tilde{l}^{p}: \tilde{b}_{0}^{p} v_{0}+\tilde{b}_{1}^{p} v_{1}+\ldots+\tilde{b}_{d}^{p} v_{d}-\tilde{n}_{1}^{p} v_{d+1}=0$, for $p \in\{1, \ldots, m\}$, and dual cones $\Delta_{\tilde{\tau}}$ associated with a vertex $\tilde{\tau}$. We identify the affine hyperplane $H=\left\{v_{0}=1\right\}$ with $\left(\mathbb{R}^{d+1}\right)^{*}$ and $H \cap\left(\mathbb{R}_{+}^{d+2}\right)^{*}$ with $\left(\mathbb{R}_{+}^{d+1}\right)^{*}$ and consider the decomposition induced by $\Delta_{\tilde{\gamma}_{p}}$ and $\Delta_{\tilde{\tau}}$ in $\left(\mathbb{R}_{+}^{d+1}\right)^{*}$. This decomposition has the same properties as the initial one. It has exactly $m$ ordered $d$-dimensional planes in $H$ and $m+1$ "pieces" of dimension $(d+1)$ between them. Let us describe them better. 
For $q=1, \ldots, r$, and $j \in\{1, \ldots, u(q)\}$ define the cone $\Delta_{q, j}^{t}$ as the intersection with $\mathbb{R}_{>0}^{d+1}$ of the hyperplane $l_{t}^{q, j}$ with integral equation:

$$
s_{1}^{q, j} \eta_{q}\left(v_{1}, \ldots, v_{d}, v_{d+1}\right)+\alpha_{q, j}=0, \text { see (4.5) }
$$

Observe that $m=\sum_{q=1}^{r} u(q)$.

The $(d+1)$-dimensional convex rational polyhedra $\Delta_{s}^{t}, s=1, \ldots, m-1$, are either the region $M_{t}^{q, j}$ (that we will call of type $M$ ) contained between two parallel hyperplanes $l_{t}^{q, j}$ and $l_{t}^{q, j+1}$ or the region $N_{t}^{q}$ (of type $N$ ) contained between the hyperplanes $l_{t}^{q, u(q)}$ and $l_{t}^{q+1,1}$ for some $q=1, \ldots, m-1$. The first one $\Delta_{0}^{t}$ and the last one $\Delta_{m}^{t}$ are

$$
\begin{array}{r}
\Delta_{0}^{t}:=\left\{v \in \mathbb{R}_{>0}^{d+1}: s_{1}^{1,1} \eta_{1}\left(v_{1}, \ldots, v_{d}, v_{d+1}\right)+\alpha_{1,1}<0\right\}, \\
\Delta_{m}^{t}:=\left\{v \in \mathbb{R}_{>0}^{d+1}: s_{1}^{r, u(r)} \eta_{r}\left(v_{1}, \ldots, v_{d}, v_{d+1}\right)+\alpha_{r, u(r)}>0\right\} .
\end{array}
$$

Thus we fix the partition of $\mathbb{R}_{>0}^{d+1}$ (identified with $H$ ) as disjoint union of three distinct types of convex rational polyhedra $\Delta_{\tau}^{t}$ :

- $d$-dimensional cones $\Delta_{q, j}^{t}$ corresponding to the hyperplanes $l_{t}^{q, j}$,

- convex rational polyhedra of type $N$, and

- convex rational polyhedra of type $M$.

Following the conventions of this work the convex rational polyhedra of type $M$ or $N$ will be called vertices and anyone of the first type an edge.

Let $\boldsymbol{\varphi} \in \mathcal{L}_{0}(X) \backslash \mathcal{L}_{0}(S)$ and let $\mathbf{k}:=\mathbf{k}(\boldsymbol{\varphi})$. The order of the differential form $\omega$ is the same as for $\bar{\omega}$, i.e., $m(\mathbf{k})=\sigma_{\omega}(\mathbf{k})-\mathbf{k}$. Fix $\mathbf{k} \in \mathbb{P}^{d+1}$ and define

$$
V_{n, m}^{\mathbf{k}, t}:=\left\{\boldsymbol{\varphi} \in \mathcal{L}_{0}(X) \backslash \mathcal{L}_{0}(S): \mathbf{k}(\boldsymbol{\varphi})=\mathbf{k}, \operatorname{ord}\left(h^{t} \circ \boldsymbol{\varphi}\right)=n+\theta, \operatorname{ord}(\omega \circ \boldsymbol{\varphi})=m\right\} ;
$$

These sets are empty unless $m=m(\mathbf{k})$, then we define

$$
Z_{D L}^{\mathbf{k}, \mathcal{W}}(T):=\sum_{n \in \mathbb{N}} \mathbb{L}^{-m(\mathbf{k})} \mu_{X}\left(V_{n, m(\mathbf{k})}^{\mathbf{k}, t}\right) T^{n}
$$

For each convex rational polyhedron $\Delta_{\tau}^{t}$ in the previous partition, define

$$
Z_{D L}^{\Delta_{\tau}^{t}, \mathcal{W}}\left(h^{t}, \omega, T\right):=\sum_{\mathbf{k} \in \Delta_{\tau}^{t} \cap \mathbb{P}^{d+1}} Z_{D L}^{\mathbf{k}, \mathcal{W}}(T) .
$$

Remark 5.11. Each hyperplane $l_{q}$ is parallel to the hyperplanes $l_{t}^{q, j}$. The region associated with a vertex $\bar{\tau}_{q}$ is a cone delimited by two hyperplanes which are parallel to the hyperplanes delimiting $N_{t}^{q}$ (different from the coordinate hyperplanes).

Remark 5.12. If $h^{t}$ is $\widetilde{\mathcal{W}}$-quasi-ordinary and Newton compatible then no region of type $M$ exists.

Step 5. Newton polyhedron of $h^{t}$ as $K$-quasi-ordinary function. 
This Newton polyhedron $\Gamma^{K}\left(h^{t}\right)$ of $h^{t}$ is the projection over the hyperplane $t=0$ of the Newton polyhedron of $h^{t}$ and it coincides with $\Gamma(h)$. For $q \in\{1, \ldots, r\}$, we define

$$
\widehat{f}_{\boldsymbol{\lambda}_{q}}^{t}:=\prod_{j=1, \alpha_{q, j} \in S_{q}}^{u(q)} \widehat{f}_{q, j}^{t}, \text { where } \widehat{f}_{q, j}^{t}:=\prod_{w=1}^{w(q, j)}\left(\left(z^{n_{1}^{q}}\right)^{s_{1}^{q, j}}-\beta_{w} t^{\alpha_{q, j}}\left(x_{1}^{b_{1}^{q}} \ldots x_{d}^{b_{d}^{q}}\right)^{s_{1}^{q, j}}\right)^{m_{q, j, w}} .
$$

\subsection{Vertices of the dual decomposition.}

In the following, the aim is to compute $Z_{D L}^{\Delta_{\tau}^{t}, \mathcal{W}}\left(h^{t}, \omega, T\right)$ for $\tau$ a vertex of type $M$ or $N$ and compare it with the computations we have already done for $Z_{D L}^{\bar{\tau}}(h, \bar{\omega}, T)$, see (4.15).

Take a convex rational polyhedron $\Delta_{\tau}^{t}$ of the dual decomposition of $\mathbb{R}_{+}^{d+1}$ which belongs to any of the two types $M$ or $N$. It corresponds to a monomial $t^{\delta} \mathbf{x}^{\boldsymbol{\alpha}} z^{n}$ whose support is the intersection of two 1-dimensional compact faces of $\Gamma^{K}\left(h^{t}\right)$. The cone $\Delta_{\tau}^{t}$ is the positive region delimited by two inequalities, say

$$
\begin{aligned}
& \tilde{\eta}_{1}\left(v_{1}, \ldots, v_{d}, v_{d+1}\right)+\tilde{\alpha}_{1}>0 \\
& \tilde{\eta}_{2}\left(v_{1}, \ldots, v_{d}, v_{d+1}\right)+\tilde{\alpha}_{2}<0
\end{aligned}
$$

where $\tilde{\eta}_{1}$ and $\tilde{\eta}_{2}$ can define parallel (or not) hyperplanes. Let $\varphi \in \mathcal{L}_{0}(X) \backslash \mathcal{L}_{0}(S)$ and let $\mathbf{k}:=\mathbf{k}(\boldsymbol{\varphi})$ and $n_{\tau}(\mathbf{k}):=\alpha_{1} k_{1}+\cdots+\alpha_{d} k_{d}+n k_{d+1}$. We have ord $\left(h^{t} \circ \boldsymbol{\varphi}\right)=$ $n_{\tau}(\mathbf{k})+N(\mathbf{k})+\delta$, with $N(\mathbf{k}):=\sum_{j=1}^{d} N_{j} k_{j}$ (we are forgetting the exponent $\theta$ ).

Lemma 5.13. If $\mathbf{k} \in \Delta_{\tau}^{t}$, then

$$
\mathbb{L}^{-m(\mathbf{k})} \mu_{X}\left(V_{n_{\tau}(\mathbf{k})+\delta+N(\mathbf{k}), m(\mathbf{k})}^{\mathbf{k}, t}\right)=\mathbb{L}^{-\left(d+1+\sigma_{\omega}(\mathbf{k})\right)}(\mathbb{L}-1)^{d+1} .
$$

Proof. The measure of the cylindrical sets can be computed as in Step 2 in $\S$, Denote $n_{\tau}(\mathbf{k})+\delta+N(\mathbf{k})$ by $\bar{n}$; hence $\mu_{X}\left(V_{\bar{n}, m(\mathbf{k})}^{\mathbf{k}, t}\right)=\left[\pi_{\bar{n}}\left(V_{\bar{n}, m(\mathbf{k})}^{\mathbf{k}, t}\right)\right] \mathbb{L}^{-(\bar{n}+1)(d+1)}$. It comes from a monomial since it is a vertex then

$$
\left[\pi_{\bar{n}}\left(V_{\bar{n}, m(\mathbf{k})}^{\mathbf{k}, t}\right)\right]=(\mathbb{L}-1)^{d+1} \mathbb{L}^{(d+1) \bar{n}-\left(k_{1}+\ldots+k_{d+1}\right)} .
$$

Hence the contribution in terms of generating functions of convex rational polyhedra is written as

$$
\begin{aligned}
Z_{D L}^{\Delta_{\tau}^{t}, \mathcal{W}} & =T^{\delta}(\mathbb{L}-1)^{d+1} \mathbb{L}^{-(d+1)} \sum_{\mathbf{k} \in \Delta_{\tau}^{t} \cap \mathbb{P}^{d+1}} \mathbb{L}^{-\sigma_{\omega}(\mathbf{k})} T^{n_{\tau}(\mathbf{k})+N(\mathbf{k})}= \\
& =T^{\delta}(\mathbb{L}-1)^{d+1} \mathbb{L}^{-(d+1)} \Phi_{\Delta_{\tau}^{t}}(\mathbf{y}),
\end{aligned}
$$

where $\mathbf{y}:=\left(\mathbb{L}^{-\nu_{1}} T^{\alpha_{1}}, \ldots, \mathbb{L}^{-\nu_{d}} T^{\alpha_{d}}, \mathbb{L}^{-1} T^{n}\right)$.

For any integer linear form $\eta\left(v_{1}, \ldots, v_{d}, v_{d+1}\right)$ and any $\alpha \in \mathbb{N}$ define $\Delta_{\eta}^{\alpha}:=\mathbb{P}^{d+1} \cap$ $\{\eta+\alpha=0\}$ and $\Delta_{\eta}^{c, \alpha}:=\mathbb{P}^{d+1} \cap\{\eta+\alpha<0\}$. The indicator functions verify the identity:

$$
\left[\Delta_{\eta}^{c, \alpha}\right]=\left[\Delta_{\eta}^{c, 0}\right]-\sum_{0<\alpha^{\prime} \leq \alpha}\left[\Delta_{\eta}^{\alpha^{\prime}}\right]
$$


In paragraph 4.3 we have already used $\Delta_{\eta}^{c, 0}$ without the superscript 0 . Since $\left[\Delta_{\tau}^{t} \cap\right.$ $\left.\mathbb{P}^{d+1}\right]=\left[\Delta_{\eta_{2}}^{c, \alpha_{2}}\right]-\left(\left[\Delta_{\eta_{1}}^{c, \alpha_{1}}\right]+\left[\Delta_{\eta_{1}}^{\alpha_{1}}\right]\right)$ then

$$
\left[\Delta_{\tau}^{t} \cap \mathbb{P}^{d+1}\right]=\left(\left[\Delta_{\eta_{2}}^{c, 0}\right]-\left[\Delta_{\eta_{1}}^{c, 0}\right]\right)-\left(\sum_{0<\alpha \leq \alpha_{2}}\left[\Delta_{\eta_{2}}^{\alpha}\right]-\sum_{0<\alpha<\alpha_{1}}\left[\Delta_{\eta_{1}}^{\alpha}\right]\right)
$$

If the corresponding hyperplanes are parallel, then $\left[\Delta_{\tau}^{t} \cap \mathbb{P}^{d+1}\right]=\sum_{\alpha_{2}<\alpha<\alpha_{1}}\left[\Delta_{\eta}^{\alpha}\right]$ being $\eta=\tilde{\eta}_{1}=\tilde{\eta}_{2}$.

Since $\eta_{q}$ has integer coefficients, $\Delta_{\eta_{q}}^{c, 0}$ is a convex (simplicial) cone with the origin as vertex. But in general the vertex of the convex rational cone $\Delta_{\eta_{q}}^{c, \alpha}$ is a rational $(d+1)$-uple which may have a non-integer coordinate and then its generating function cannot be computed from the one of $\Delta_{\eta_{q}}^{c, 0}$ with an integer translation. To avoid this problem we do the following.

Let $H_{q}^{\alpha}$ be the hyperplane of equation $\eta_{q}+\alpha=0, \alpha \in \mathbb{N}$. The difference of two elements in a given $H_{q}^{\alpha}$ belongs to $l^{q} \cap \mathbb{Z}^{d+1}$. Recall that

$$
l^{q} \cap \mathbb{Z}^{d+1}=G^{q}+\mathbb{Z} \mathbf{w}_{1}^{q}+\cdots+\mathbb{Z} \mathbf{w}_{d}^{q} \quad \text { and } \quad l^{q} \cap \mathbb{P}^{d+1}=G^{q}+\mathbb{N w}_{1}^{q}+\cdots+\mathbb{N}_{d}^{q},
$$

where $\Delta_{\eta_{q}}^{0}=l^{q} \cap \mathbb{P}^{d+1}$ and $G^{q}$ is the fundamental set of $\Delta_{\eta_{q}}^{0}$ :

$$
G^{q}:=\mathbb{N}^{d} \cap\left\{\sum_{l=1}^{d} \mu_{l} \mathbf{w}_{l}^{q}: 0<\mu_{l} \leq 1 \text { for } l=1, \ldots, d\right\} .
$$

We need the following lemma which we will prove later.

Lemma 5.14. For every $\alpha \in \mathbb{N}$, there exists a subset $G^{q, \alpha} \subset H_{q}^{\alpha}$ with a bijection between $G^{q}$ and $G^{q, \alpha}$ such that $H_{q}^{\alpha} \cap \mathbb{P}^{d+1}=G^{q, \alpha}+\mathbb{N w}_{1}^{q}+\cdots+\mathbb{N w}_{d}^{q}$.

In particular for any $\mathbf{k} \in \Omega^{q, \alpha}$ there exists a unique $\mathbf{g} \in G^{q, \alpha}$ and $\left(l_{1}, \ldots, l_{d}\right) \in \mathbb{N}^{d}$ such that $\mathbf{k}=\mathbf{g}+l_{1} \mathbf{w}_{1}^{q}+\cdots+l_{d} \mathbf{w}_{d}^{q}$.

As a consequence of equation (3.1), for every $\alpha \in \mathbb{N}$, we have

$$
\Phi_{\Delta_{\eta_{q}}^{\alpha}}(\mathbf{y})=\frac{\left(\sum_{\mathbf{g} \in G^{q, \alpha}} \mathbf{y}^{\mathbf{g}}\right)}{\prod_{l=1}^{d}\left(1-\mathbf{y}^{\mathbf{w}_{l}^{q}}\right)}
$$

Assume $\Delta_{\tau}^{t}$ is of type $N$ and is the region delimited by the inequalities $\eta_{q}+\alpha_{q, u(q)}>$ $0, \eta_{q+1}+\alpha_{q+1,1}<0$. Assume also that $\Delta_{\tau}^{t}$ is different from $\Delta_{m}^{t}$ and $\Delta_{0}^{t}$ (these will be studied later on). The associated vertex $\bar{\tau}$ of $\Gamma(h)$ gives a convex cone $\Delta_{\bar{\tau}}$ delimited by the inequalities $\eta_{q}>0, \eta_{q+1}<0$. Thus the monomial $\overline{\mathbf{x}}^{\boldsymbol{\alpha}} \bar{z}^{n}$ appears in the sum defining $h$. Moreover if $\mathbf{k} \in \Delta_{\bar{\tau}}$ and $\mathbf{k}(\boldsymbol{\varphi})=\mathbf{k}$ then $\operatorname{ord}(h \circ \boldsymbol{\varphi})=n_{\tau}(\mathbf{k})+N(\mathbf{k})$. As in lemma 5.13, $\mathbb{L}^{-m(\mathbf{k})} \mu_{X}\left(V_{n_{\tau}(\mathbf{k})+N(\mathbf{k}), m(\mathbf{k})}^{\mathbf{k}}\right)=\mathbb{L}^{-(d+1)}(\mathbb{L}-1)^{d+1} \mathbb{L}^{-\sigma_{\bar{\omega}}(\mathbf{k})}$.

In this case we can be more explicit in equality (5.6) because $\left[\Delta_{\eta_{q+1}}^{c, 0}\right]-\left[\Delta_{\eta_{q}}^{c, 0}\right]=$ $\left[\Delta_{\bar{\tau}_{q}}\right]+\left[\Delta_{\bar{\gamma}_{q}}\right]$, see equation (4.10). Moreover both generating functions are evaluated 
at the same value $\mathbf{y}:=\left(\mathbb{L}^{-\nu_{1}} T^{\alpha_{1}}, \ldots, \mathbb{L}^{-\nu_{d}} T^{\alpha_{d}}, \mathbb{L}^{-1} T^{n}\right)$. Thus

$Z_{D L}^{\Delta_{\tau}^{t}, \mathcal{W}}(T)=T^{\delta}(\mathbb{L}-1)^{d+1} \mathbb{L}^{-(d+1)}\left(\Phi_{\Delta_{\bar{\tau}_{q}}}(\mathbf{y})+\sum_{0<\alpha \leq \alpha_{2}} \Phi_{\Delta_{\eta_{q+1}}^{\alpha}}(\mathbf{y})-\sum_{0 \leq \alpha<\alpha_{1}} \Phi_{\Delta_{\eta_{q}}^{\alpha}}(\mathbf{y})\right)$

Lemma 5.15. For each $\Delta_{\tau}^{t}$ of type $N$ its contribution to the topological zeta function is $\chi_{\text {top }}\left(Z_{D L}^{\Delta_{r}^{t}, \mathcal{W}}\left(h^{t}, \omega, \mathbb{L}^{-s}\right)\right)=\chi_{\text {top }}\left(Z_{D L}^{\bar{\tau}}\left(h, \bar{\omega}, \mathbb{L}^{-s}\right)\right)$.

Proof. The proof follows directly first from the description in equation (15.7) and the fact that in the denominator of any $\Phi_{\Delta_{\eta}^{\alpha}}(\mathbf{y})$ there are at most $d$ factors.

Remark 5.16. This fact will be used for both theorems [5.3 and 5.9.

The indicator function $\left[\Delta_{0}^{t}\right]$ for the first convex rational polyhedron $\Delta_{0}^{t}=\left\{\eta_{1}+\right.$ $\left.\alpha_{1,1}<0\right\}$ also verifies the identity $\left[\Delta_{0}^{t}\right]=\left[\Delta_{\eta_{1}}^{c, 0}\right]-\sum_{0<\alpha \leq \alpha_{1,1}}\left[\Delta_{\eta_{1}}^{\alpha}\right]$. In particular one has for this convex polyhedron a lemma similar to Lemma 5.15 too.

For the last convex rational polyhedron $\Delta_{m}^{t}=\left\{\eta_{r}+\alpha_{r, u(r)}>0\right\},\left[\Delta_{m}^{t}\right]=\left[\Delta_{\eta_{r}}^{c, 0}\right]+$ $\sum_{0 \leq \alpha<\alpha_{r, u(r)}}\left[\Delta_{\eta_{r}}^{\alpha}\right]$. Thus

$$
Z_{D L}^{\Delta_{m}^{t}, \mathcal{W}}\left(h^{t}, \omega, T\right)=T^{\delta}(\mathbb{L}-1)^{d+1} \mathbb{L}^{-(d+1)}\left(\Phi_{\Delta_{\bar{\tau}_{r}}}(\mathbf{y})+\sum_{0 \leq \alpha<\alpha_{r, u(r)}} \Phi_{\Delta_{\eta_{r}}^{\alpha}}(\mathbf{y})\right)
$$

And again one gets for this polyhedron a lemma similar to Lemma 5.15 .

If the convex rational polyhedron $\Delta_{\tau}^{t}$ is of type $M$ limited by two parallel hyperplanes, say for instance $l_{q, j}^{t}$ and $l_{q, j+1}^{t}$, then $\Delta_{\tau}^{t}$ also comes from a vertex, say $t^{\delta} \mathbf{x}^{\boldsymbol{\alpha}} z^{n}$. Consider $\bar{\gamma}_{q}$ the corresponding compact face of $\Gamma(h)$ such that it defines $l^{q}$ in the dual. One of the vertices of $\bar{\gamma}_{q}$ has to be $\overline{\mathbf{x}}^{\boldsymbol{\alpha}} \bar{z}^{n}$ because it is connected with one of the vertices of type $N$. We have already mention that $\left[\Delta_{\tau}^{t} \cap \mathbb{P}^{d+1}\right]$ is nothing but $\sum_{\alpha_{q, j+1}<\alpha<\alpha_{q, j}}\left[\Delta_{\eta_{q}}^{\alpha}\right]$. Then

$$
Z_{D L}^{\Delta_{\tau}^{t}, \mathcal{W}}\left(h^{t}, \omega, T\right)=T^{\delta}(\mathbb{L}-1)^{d+1} \mathbb{L}^{-(d+1)}\left(\sum_{\alpha_{q, j+1}<\alpha<\alpha_{q, j}} \Phi_{\Delta_{\eta_{q}}^{\alpha}}(\mathbf{y})\right)
$$

where $\mathbf{y}:=\left(\mathbb{L}^{-\nu_{1}} T^{\alpha_{1}}, \ldots, \mathbb{L}^{-\nu_{d}} T^{\alpha_{d}}, \mathbb{L}^{-1} T^{n}\right)$. We observe that $\left(\alpha_{1}, \ldots, \alpha_{d}, n\right)$ also belongs to the closure of $\bar{\gamma}_{q}$. Therefore after identity (5.7) we have proved the next proposition.

Proposition 5.17. If $\Delta_{\tau}^{t}$ is of type $M$, then $\chi_{\text {top }}\left(Z_{D L}^{\Delta_{\tau}^{t}, \mathcal{W}}\left(h^{t}, \omega, \mathbb{L}^{-s}\right)\right)=0$.

We summarize the previous results and we collect also the information about the denominators of the partial motivic zeta functions, using the above arguments and the description of the corresponding cones given in lemmas 4.14, 4.15, 4.13 and 4.14,

Proposition 5.18. For each vertex $\bar{\tau} \in \Gamma(h)$ and each of the vertices $\Delta_{\tau}^{t}$ of type $M$ or $N$ of $\Gamma^{t}\left(h^{t}\right)$, the functions $Z_{D L}^{\Delta_{\tau}^{t}, \mathcal{W}}\left(h^{t}, \omega, T\right)$ and $Z_{D L}^{\bar{\tau}}(h, \bar{\omega}, T)$ belong to the subring $\mathbb{Z}\left[\mathbb{L}, \mathbb{L}^{-1},\left(1-\mathbb{L}^{-1} T\right)^{-1},\left(1-\mathbb{L}^{-\sigma_{\omega}\left(\mathbf{w}_{j}^{q}\right)} T^{m_{h}\left(\mathbf{w}_{j}^{q}\right)}\right)^{-1},\left(1-\mathbb{L}^{-\nu_{i}} T^{N_{i}}\right)^{-1}\right][T], j, i \in\{1, \ldots, d\}$, and $q=1, \ldots, r$. 
There is a one-to-one correspondence between elements of type $N$ and vertices of $\Gamma(h)$. If $\Delta_{\tau}^{t}$ is of type $N$ and under this bijection corresponds with $\bar{\tau} \in \Gamma(h)$ then $\chi_{\text {top }}\left(Z_{D L}^{\Delta_{\tau}^{t}, \mathcal{W}}\left(h^{t}, \omega, \mathbb{L}^{-s}\right)\right)=\chi_{\text {top }}\left(Z_{D L}^{\bar{\tau}}\left(h, \bar{\omega}, \mathbb{L}^{-s}\right)\right)$.

Moreover, if $\Delta_{\tau}^{t}$ is of type $M$ then $\chi_{\mathrm{top}}\left(Z_{D L}^{\Delta_{\tau}^{t}, \mathcal{W}}\left(h^{t}, \omega, \mathbb{L}^{-s}\right)\right)=0$.

Proof of Lemma 5.14. Let $\mathbf{h} \in H_{q}^{\alpha} \cap \mathbb{Z}^{d+1}$, and let $\mathbf{g} \in G^{q}$. Write

$$
\mathbf{h}+\mathbf{g}=\left(h_{1}, h_{2}, \ldots, h_{d}, h_{d+1}\right) .
$$

Using the division algorithm, for $l=1, \ldots, d+1$, we decompose $h_{l}=s_{l} p_{l}^{q}+r_{l}$, where $s_{l} \in \mathbb{Z}$ and $0<r_{l} \leq p_{l}^{q}$. Since $\mathbf{h} \in H_{q}^{\alpha}$ and $\mathbf{g} \in l^{q}$, then $\mathbf{h}+\mathbf{g} \in H_{q}^{\alpha}$. This implies that

$$
\sum_{l=1}^{d} b_{l}^{q} r_{l}+\alpha=h_{d+1} n_{1}^{q}-\sum_{l=1}^{d} b_{l}^{q} s_{l} p_{l}^{q}=\left(h_{d+1}-\sum_{l=1}^{d} \bar{b}_{l}^{q} s_{l}\right) n_{l}^{q}, \text { i.e. } h_{d+1}-\sum_{l=1}^{d} \bar{b}_{l}^{q} s_{l} \in \mathbb{P} .
$$

Let us denote

$$
\mathbf{h}_{\mathrm{g}}^{1}=\left(r_{1}, \ldots, r_{d}, h_{d+1}-\sum_{l=1}^{d} \bar{b}_{l}^{q} s_{l}\right) \in H_{q}^{\alpha} \cap \mathbb{P}^{d+1} .
$$

We have $\mathbf{h}+\mathbf{g}=\mathbf{h}_{\mathbf{g}}^{1}+s_{1} \mathbf{w}_{1}^{q}+\cdots+s_{d} \mathbf{w}_{d}^{q}$.

Let $\mathbf{h}^{\prime} \in H_{q}^{\alpha} \cap \mathbb{P}^{d+1}$. Then $\mathbf{h}^{\prime}-\mathbf{h} \in l^{q} \cap \mathbb{Z}^{d+1}$. There exist $\mathbf{g} \in G^{q}$ and $\left(u_{1}, \ldots, u_{d}\right) \in$ $\mathbb{Z}^{d}$ such that

$$
\mathbf{h}^{\prime}=\mathbf{h}+\mathbf{g}+u_{1} \mathbf{w}_{1}^{q}+\cdots+u_{d} \mathbf{w}_{d}^{q} \text {, i.e. } \mathbf{h}^{\prime}=\mathbf{h}_{\mathbf{g}}^{1}+\sum_{l=1}^{d}\left(u_{l}-s_{l}\right) \mathbf{w}_{l}^{q} .
$$

If $\mathbf{h}^{\prime}=\left(h_{1}^{\prime}, \ldots, h_{d}^{\prime}, h_{d+1}^{\prime}\right)$ then, for $l=1, \ldots, d$, we have $h_{l}^{\prime}=r_{l}+\left(u_{l}-s_{l}\right) p_{l}^{q}$.

Since $h_{l}^{\prime} \in \mathbb{P}$ and $0<r_{l} \leq p_{l}^{q}$, then $\left(u_{l}-s_{l}\right) \in \mathbb{N}$. We define

$$
G^{q, \alpha}:=\left\{\mathbf{h}_{\mathrm{g}}^{1} \mid \mathbf{g} \in G^{q}\right\} .
$$

We have proved that $H_{q}^{\alpha} \cap \mathbb{N}_{>0}^{d+1}=G^{q, \alpha}+\mathbb{N w}_{1}^{q}+\cdots+\mathbb{N w}_{d}^{q}$.

It is enough to prove that $G^{q}$ and $G^{q, \alpha}$ are bijective. Now let us suppose that $\mathbf{h}_{\mathbf{g}}^{1}=\mathbf{h}_{\mathbf{g}^{\prime}}^{1}$ for some $\mathbf{g}, \mathbf{g}^{\prime} \in G^{q}$. We can write:

$$
\mathbf{h}+\mathbf{g}=\mathbf{h}_{\mathbf{g}}^{1}+\sum_{l=1}^{d} s_{l} \mathbf{w}_{l}^{q} \text { and } \mathbf{h}+\mathbf{g}^{\prime}=\mathbf{h}_{\mathbf{g}^{\prime}}^{1}+\sum_{l=1}^{d} s_{l}^{\prime} \mathbf{w}_{l}^{q} .
$$

We have $\mathbf{g}-\mathbf{g}^{\prime}=\sum_{l=1}^{d}\left(s_{l}-s_{l}^{\prime}\right) \mathbf{w}_{l}^{q} \in l^{q} \cap \mathbb{Z}^{d+1}$. Since the elements of this set can be written in a unique form as elements of $G^{q}+\mathbb{Z} \mathbf{w}_{1}^{q}+\cdots+\mathbb{Z} \mathbf{w}_{d}^{q}$ then $\mathbf{g}=\mathbf{g}^{\prime}$.

\subsection{Edges of the Newton polytope.}

We now deal with edges. Consider the edges $\bar{\gamma}_{1}, \ldots, \bar{\gamma}_{r}$ of $\Gamma(h)$. Fix $q=1, \ldots, r$. Since $f_{\boldsymbol{\lambda}_{q}}=\prod_{\bar{j}=1}^{v(q)}\left(z^{n_{1}^{q}}-\bar{\beta}_{\bar{j}}^{q} \mathbf{x}^{n_{1}^{q} \boldsymbol{\lambda}_{q}}\right)^{m_{q, j}}$ is the quasihomogeneous part of $f$ corresponding to this face, $\left[N_{\bar{\gamma}_{q}}\right]=\left[\mathbf{G}_{m, k}^{d+1} \cap\left\{f_{\boldsymbol{\lambda}_{q}}=0\right\}\right]=v(q)(\mathbb{L}-1)^{d}$, see Remark 4.18, 
In Step [5, for each $j \in\{1, \ldots, u(q)\}$, the polynomial $\widehat{f}_{q, j}^{t}:=\prod_{w=1}^{w(q, j)}\left(\left(z^{n_{1}^{q}}\right)^{s_{1}^{q, j}}-\right.$ $\left.\beta_{w} t^{\alpha_{q, j}}\left(\mathbf{x}^{n_{1}^{q} \boldsymbol{\lambda}_{\kappa, q}}\right)^{s_{1}^{q, j}}\right)^{m_{q, j, w}}$ was defined. This polynomial is associated with a region $M_{t}^{q, j}$ which is contained in a hyperplane parallel to $l^{q}$. Next result is trivial because under the hypothesis of the proposition there is no points with positive integer coordinates in $M_{t}^{q, j}$.

Proposition 5.19. If $s_{1}^{q, j}>1$ then $M_{t}^{q, j} \cap \mathbb{N}^{d+1}=\emptyset$ and $Z_{D L}^{M_{t}^{q, j}, \mathcal{W}}\left(h^{t}, \omega, T\right)=0$.

From now on we will suppose $s_{1}^{q, j}=1$. Because of this proposition we cannot state the equality for the topological zeta function in Theorem 5.3. For the other edges, following the notation in $\oiint 3$ we break the sets of $\operatorname{arcs}$ in $A$ - and $B$-parts. We will consider,

$$
\widehat{f}_{q, j \mid t=1}^{t}:=\prod_{w=1}^{w(q, j)}\left(z^{n_{1}^{q}}-\beta_{w} \mathbf{x}^{n_{1}^{q} \boldsymbol{\lambda}_{\kappa, q}}\right)^{m_{q, j, w}} .
$$

Remark 5.20. Observe that for $\widetilde{\mathcal{W}}$-quasi-ordinary and Newton compatible series, for each $q$ we have $u(q)=1$. The extra hypothesis $s_{1}^{q, j}=1$ is fulfilled by $\widetilde{\mathcal{W}}$-quasiordinary and Newton compatible series and this is why equality for topological zeta functions may be obtained in Theorem 5.9.

Step 6. Edges in the A-part.

Take a convex rational cone $\Delta_{q, j}^{t}$ corresponding to an edge. We decompose its contribution into two disjoint parts as in the non-degenerated case. Given $\mathbf{k} \in \Delta_{q, j}^{t}$, we set:

$$
\begin{aligned}
V_{n, A, m}^{\mathbf{k}, t}:=\left\{\boldsymbol{\varphi} \in \mathcal{L}_{0}(X) \backslash \mathcal{L}_{0}(S):\right. \\
\left.\quad \mathbf{k}(\boldsymbol{\varphi})=\mathbf{k}, \operatorname{ord}\left(h^{t} \circ \boldsymbol{\varphi}\right)=n+\theta, \operatorname{ord}(\omega \circ \boldsymbol{\varphi})=m, \widehat{f}_{q, j \mid t=1}^{t}(\mathbf{a}(\boldsymbol{\varphi})) \neq 0\right\} .
\end{aligned}
$$

These semialgebraic subsets are empty unless $m=m(\mathbf{k})$ and $n=\eta_{q}(\mathbf{k})+\alpha_{q, j}+N(\mathbf{k})$. In particular $\left[\mathbf{G}_{m, k}^{d+1} \cap\left\{\widehat{f}_{q, j \mid t=1}^{t}=0\right\}\right]=w(q, j)(\mathbb{L}-1)^{d} \in K_{0}\left(\operatorname{Var}_{k}\right)$, see Remark 4.18.

Let us (re)define

$$
Z_{q, j, A}^{\mathcal{W}}\left(h^{t}, \omega, T\right):=\sum_{\mathbf{k} \in \Delta_{q, j}^{t}} \mathbb{L}^{-m(\mathbf{k})} \mu_{X}\left(V_{\hat{n}_{q}(\mathbf{k})+\alpha_{q, j}+N(\mathbf{k}), A, m(\mathbf{k})}^{\mathbf{k}, t}\right) T^{\eta_{q}(\mathbf{k})+\alpha_{q, j}+N(\mathbf{k})} .
$$

In the same way, given $\mathbf{k} \in \Delta_{\bar{\gamma}_{q}}$ we set:

$$
\begin{aligned}
V_{n, A, m}^{\mathbf{k}}:=\left\{\boldsymbol{\varphi} \in \mathcal{L}_{0}(X) \backslash \mathcal{L}_{0}(S):\right. \\
\left.\quad \mathbf{k}(\boldsymbol{\varphi})=\mathbf{k}, \operatorname{ord}(h \circ \boldsymbol{\varphi})=n, \operatorname{ord}(\omega \circ \boldsymbol{\varphi})=m, f_{\boldsymbol{\lambda}_{\kappa, q}}(\mathbf{a}(\boldsymbol{\varphi})) \neq 0\right\} .
\end{aligned}
$$

Again the semialgebraic subsets are empty unless $m=m(\mathbf{k})$ and $n=\eta_{q}(\mathbf{k})+N(\mathbf{k})$. We recall that

$$
Z_{\bar{\gamma}_{q}, A}(h, \bar{\omega}, T)=\sum_{\mathbf{k} \in \Delta_{\bar{\gamma}_{q}}} \mathbb{L}^{-m(\mathbf{k})} \mu_{X}\left(V_{\hat{n}_{q}(\mathbf{k})+N(\mathbf{k}), A, m(\mathbf{k})}^{\mathbf{k}}\right) T^{\eta_{q}(\mathbf{k})+N(\mathbf{k})} .
$$


Lemma 5.21. For each $q=1, \ldots, r, j=1, \ldots, u(q)$, the functions $Z_{q, j, A}^{\mathcal{W}}\left(h^{t}, \omega, T\right)$ and $Z_{\bar{\gamma}_{q}, A}(h, \bar{\omega}, T)$ belong to the subring $\mathbb{Z}\left[\mathbb{L}, \mathbb{L}^{-1},\left(1-\mathbb{L}^{-\sigma_{\omega}\left(\mathbf{w}_{l}^{q}\right)} T^{m_{h}\left(\mathbf{w}_{l}^{q}\right)}\right)^{-1}\right][T], l=$ $1, \ldots, d$. Moreover, the following equality holds:

$$
\sum_{j=1}^{u(q)} \chi_{\text {top }}\left(Z_{q, j, A}^{\mathcal{W}}\left(h^{t}, \omega, \mathbb{L}^{-s}\right)\right)=\chi_{\text {top }}\left(Z_{\bar{\gamma}_{q}, A}\left(h, \bar{\omega}, \mathbb{L}^{-s}\right)\right) .
$$

Proof. We can follow the proofs of Theorem 3.4. Lemma 5.13 and Lemma 5.15, Since we are in the $A$-part of the decomposition, the measure of the sets $V_{\eta_{q}(\mathbf{k})+N(\mathbf{k}), A, m(\mathbf{k})}^{\mathbf{k}}$ and $V_{\eta_{q}(\mathbf{k})+\alpha_{q, j}+N(\mathbf{k}), A, m(\mathbf{k})}^{\mathbf{k}, t}$ can be explicitly computed. Since $h^{t}$ and $h$ has the same characteristic exponents then the Newton polyhedra $\Gamma^{K}\left(h^{t}\right)$ and $\Gamma(h)$ coincide and we get a sum as (5.9) but with only one $\alpha=\alpha_{q, j}$. Then

$$
Z_{q, j, A}^{\mathcal{W}}\left(h^{t}, \omega, T\right)=\mathbb{L}^{-(d+1)}\left((\mathbb{L}-1)^{d+1}-w(q, j)(\mathbb{L}-1)^{d}\right) T^{\alpha_{q, j}} \Phi_{\Delta_{\eta_{q}}^{\alpha_{q, j}}}(\mathbf{y}),
$$

where $\mathbf{y}:=\left(\mathbb{L}^{-\nu_{1}} T^{\alpha_{1}}, \ldots, \mathbb{L}^{-\nu_{d}} T^{\alpha_{d}}, \mathbb{L}^{-1} T^{n}\right)$ and $\left(\alpha_{1}, \ldots, \alpha_{d}, n\right)$ is an element in the closure of $\bar{\gamma}$. In the same way

$$
Z_{\bar{\gamma}_{q}, A}(h, \bar{\omega}, T)=\mathbb{L}^{-(d+1)}\left((\mathbb{L}-1)^{d+1}-v(q)(\mathbb{L}-1)^{d}\right) \Phi_{\Delta_{\eta_{q}}^{\alpha_{q, j}}}(\mathbf{y}) .
$$

Since there is a bijection between the sets $G^{q}$ and $G^{q, \alpha_{q, j}}$ and $v(q)=\sum w(q, j)$ for $j=1, \ldots, u(q)$ then taking the usual Euler characteristic the lemma is proved.

To sum up we collect the $A$-part in the arc decomposition as follows:

$$
\begin{array}{r}
Z_{D L}^{A}(h, \bar{\omega}, T)=\sum_{\bar{\tau} \text { vertex }} Z_{D L}^{\bar{\tau}}(h, \bar{w}, T)+\sum_{q=1}^{r} Z_{\bar{\gamma}_{q}, A}(h, \bar{\omega}, T), \\
Z_{A}^{\mathcal{W}}\left(h^{t}, \omega, T\right):=\sum_{\Delta_{\tau}^{t} \text { vertex }} Z_{D L}^{\Delta_{\tau}^{t}, \mathcal{W}}\left(h^{t}, \omega, T\right)+\sum_{q=1}^{r} \sum_{j=1}^{u(q)} Z_{q, j, A}^{\mathcal{W}}\left(h^{t}, \omega, T\right) .
\end{array}
$$

Remark 5.22. Up to now we have proved two facts:

- For a $\mathcal{W}$-quasi-ordinary power series the denominators of the $A$-part of the motivic zeta function are controlled.

- For a $\widehat{\mathcal{W}}$-quasi-ordinary power series which is Newton compatible, identity (4.16) also holds.

We need to replace $k$ by $k[[t]]$ because of computations of the $B$-part. Observe that in the $B$-part there are no vertices.

Step 7. Edges in the $B$-part.

Given $\mathbf{k} \in \Delta_{q, j}^{t}$, consider the semialgebraic subsets

$$
\begin{aligned}
V_{n, B, m}^{\mathbf{k}, t}:=\left\{\boldsymbol{\varphi} \in \mathcal{L}_{0}(X) \backslash \mathcal{L}_{0}(S):\right. \\
\left.\quad \mathbf{k}(\boldsymbol{\varphi})=\mathbf{k}, \operatorname{ord}\left(h^{t} \circ \boldsymbol{\varphi}\right)=n+\theta, \operatorname{ord}(\omega \circ \boldsymbol{\varphi})=m, \widehat{f}_{q, j \mid t=1}^{t}(\mathbf{a}(\boldsymbol{\varphi}))=0\right\} .
\end{aligned}
$$


If $\mathbf{k} \in \Delta_{\bar{\gamma}_{q}}$ we define $V_{n, B, m}^{\mathbf{k}}$ in the same way. We have to compute $Z_{q, j, B}^{\mathcal{W}}\left(h^{t}, \omega, T\right)$ and $Z_{\bar{\gamma}_{q}, B}(h, \bar{\omega}, T)$ which have obvious definitions.

The sets $\Delta_{\bar{\gamma}_{q}} \cap \mathbb{P}^{d+1}$, resp. $\Delta_{q, j}^{t} \cap \mathbb{P}^{d+1}$, are the disjoint unions of the sets $\mathbf{g}+$ $\mathbf{w}_{1}^{q} \mathbb{N}+\ldots+\mathbf{w}_{d}^{q} \mathbb{N}$ where $\mathbf{g} \in G^{q}$, resp. $\mathbf{g} \in G^{q, \alpha_{q, j}}$. Accordingly, we define the sets

$$
V_{n, B, m}^{\bar{\gamma}_{q}, \mathbf{g}}:=\bigcup_{\mathbf{k} \in \mathbf{g}+\mathbf{w}_{1}^{q} \mathbb{N}+\ldots+\mathbf{w}_{d}^{q} \mathbb{N}} V_{n, B, m}^{\mathbf{k}}, \quad \mathbf{g} \in G^{q},
$$

and

$$
V_{n, B, m}^{q, j, \mathbf{g}, t}:=\bigcup_{\mathbf{k} \in \mathbf{g}+\mathbf{w}_{1}^{q} \mathbb{N}+\ldots+\mathbf{w}_{d}^{q} \mathbb{N}} V_{n, B, m}^{\mathbf{k}, t}, \quad \mathbf{g} \in G^{q, \alpha_{q, j}} .
$$

Let $\boldsymbol{\varphi} \in V_{n, B, m}^{\bar{\gamma}_{q}, \mathbf{g}}$, with $\mathbf{g} \in G^{q}$; this arc is related to exactly one Newton component of $f$, see subsection 4.4. There is a unique $\bar{j}=1, \ldots, v(q)$ such that $\mathbf{a}(\boldsymbol{\varphi}) \in \mathbf{G}_{m, k}^{d+1}$ belongs to the zero locus $V_{\bar{j}}^{q} \subset \mathbb{A}_{k}^{d+1}$ of the quasi-ordinary polynomial $f_{q, j}=z^{n_{1}^{q}}-$ $\bar{\beta} \frac{q}{j} \mathbf{x}^{n_{1}^{q} \boldsymbol{\lambda}_{q}}$.

Remark 5.23. Let $J_{q}$ be the subset of $\{1, \ldots, d\}$ of the non-zero coordinates of $\boldsymbol{\lambda}_{q}$. It may happen that $J_{q} \varsubsetneqq\{1, \ldots, d\}$. For the sake of simplicity we suppose that there is equality and we will point out where the non-equality may affect.

We decompose the set $V_{n, B, m}^{\bar{\gamma}_{q}, \mathbf{g}}$ in $v(q)$ disjoint sets according to this property:

$$
V_{n, B, m}^{\bar{\gamma}_{q}, \mathbf{g}}=\bigcup_{\bar{j}=1}^{v(q)} V_{n, B, m}^{\bar{\gamma}_{q}, \mathbf{g}, \bar{j}} .
$$

In the same way the sets $V_{n, B, m}^{t, q, j, \mathbf{g}, w}, w=1, \ldots, w(q, j)$, are defined. It is also possible to decompose

$$
V_{n, B, m}^{\bar{\gamma}_{q}, \mathbf{g}, \bar{j}}=\bigcup_{\mathbf{r}:=\left(r_{1}, \ldots, r_{d}\right) \in \mathbb{N}^{d}} V_{n, B, m}^{\bar{\gamma}_{q}, \mathbf{g}, j, \mathbf{r}},
$$

where $V_{n, B, m}^{\bar{\gamma}_{q}, \mathbf{g}, \bar{j}, \mathbf{r}}:=\left\{\boldsymbol{\varphi} \in V_{n, B, m}^{\bar{\gamma}_{q}, \mathbf{g}, \bar{j}}: \mathbf{k}(\boldsymbol{\varphi})=\mathbf{g}+r_{1} \mathbf{w}_{1}^{q}+\ldots+r_{d} \mathbf{w}_{d}^{q}\right\}$, and consider in the same way $V_{n, B, m}^{t, q, j, \mathbf{g}, w, \mathbf{r}}$. Define

$$
Z^{\bar{\gamma}_{q}, \mathbf{g}, \bar{j}, \mathbf{r}}(T):=\sum_{n \geq 1}\left(\sum_{m \geq 1} \mathbb{L}^{-m} \mu_{X}\left(V_{n, B, m}^{\bar{\gamma}_{q}, \mathbf{g}, \bar{j}, \mathbf{r}}\right)\right) T^{n},
$$

and also $Z_{q, j}^{\mathcal{W}, \mathbf{g}, w, \mathbf{r}}(T)$. We define $Z^{\bar{\gamma}_{q}, \mathbf{g}, j}(T):=\sum_{\mathbf{r} \in \mathbb{N}^{d}} Z^{\bar{\gamma}_{q}, \mathbf{g}, \bar{j}, \mathbf{r}}(T)$ and the correspond$\operatorname{ing} Z_{q, j}^{\mathcal{W}, \mathbf{g}, w}(T)$; then we have the decompositions:

$$
\begin{array}{r}
Z_{D L}^{\mathcal{W}}\left(h^{t}, \omega, T\right)=Z_{A}^{\mathcal{W}}\left(h^{t}, \omega, T\right)+\sum_{q=1}^{r} \sum_{j=1}^{u(q)} \sum_{\mathbf{g} \in G^{q, \alpha} q, j} \sum_{w=1}^{w(q, j)} Z_{q, j}^{\mathcal{W}, \mathbf{g}, w}(T) \\
Z_{D L}(h, \bar{\omega}, T)=Z_{D L}^{A}(h, \bar{\omega}, T)+\sum_{q=1}^{r} \sum_{\mathbf{g} \in G^{q}} \sum_{\bar{j}=1}^{v(q)} Z^{\bar{\gamma}_{q}, \mathbf{g}, \bar{j}}(T),
\end{array}
$$


where $v(q)=\sum_{j=1}^{u(q)} w(q, j)$. Using Proposition 5.18 and Lemma 5.21 we have proved that $Z_{A}^{\mathcal{W}}\left(h^{t}, \omega, T\right)$ verifies Theorems 5.3 and 5.9 in their respective cases. Then we have to prove them for arcs in the $B$-part of the decomposition. In particular we will describe in the following steps the computations in the identities of (5.13).

Step 8. Computations for $h$ and $\mathbf{g}=\mathbf{g}^{1}:=\mathbf{w}_{1}^{q}+\ldots+\mathbf{w}_{d}^{q} \in G^{q}$, and $\bar{j}=1, \ldots, v(q)$.

We use the notation of subsection 4.4. To compute $Z^{\bar{\gamma}_{q}, \mathbf{g}^{1}, \bar{j}, \mathbf{r}}(T)$, for $\mathbf{r} \in \mathbb{N}^{d}$, then $\mathbf{k}=\left(r_{1}+1\right) \mathbf{w}_{1}^{q}+\ldots+\left(r_{d}+1\right) \mathbf{w}_{d}^{q}$ has coordinates $k_{l}=\left(r_{l}+1\right) p_{l}^{q}$ with $l=1, \ldots, d$, and $k_{d+1}=\sum_{l=1}^{d}\left(r_{l}+1\right) \bar{b}_{l}^{q}$. If $\boldsymbol{\varphi}$ verifies that $\mathbf{k}(\boldsymbol{\varphi})=\mathbf{k}$ then $m_{\mathbf{k}}:=\operatorname{ord}(\bar{\omega} \circ \boldsymbol{\varphi})=$ $\sum_{l=1}^{d}\left(r_{l}+1\right) p_{l}^{q}\left(\nu_{l}-1\right)$. Therefore $m_{\mathbf{k}}$ is the unique value of $m$ for which $V_{n, B, m}^{\bar{\gamma}_{q}, \mathbf{g}^{1}, \bar{j}, \mathbf{r}}$ may be non-empty. Hence

$$
Z^{\bar{\gamma}_{q}, \mathbf{g}^{1}, \bar{j}, \mathbf{r}}(T)=\sum_{n \geq 1} \mathbb{L}^{-m_{\mathbf{k}}} \mu_{X}\left(V_{n, B, m_{\mathbf{k}}}^{\bar{\gamma}_{q}, \mathbf{g}^{1}, \bar{j}, \mathbf{r}}\right) T^{n}
$$

Fix the parametrization $\pi_{\frac{j}{j}}^{q}: \mathbb{A}_{k}^{d} \rightarrow V_{\bar{j}}^{q}$ as in subsection 4.4. Take $\varphi \in V_{n, B, m_{\mathbf{k}}}^{\bar{\gamma}_{q}, \mathbf{g}^{1}, \bar{j}, \mathbf{r}}$ and the unique $\mathbf{s}^{0} \in \mathbf{G}_{m, k}^{d}$ such that $\pi_{j}^{q}\left(\mathbf{s}^{0}\right)=\mathbf{a}(\boldsymbol{\varphi})$. The idea is to lift $\boldsymbol{\varphi}$ to an affine space $Y:=\mathbb{A}_{k}^{d+1}$ with coordinates $\left(\mathbf{y}, z_{1}\right)$ using the Newton map $\pi_{q, \bar{j}}$ associated with $f_{\frac{q}{j}}^{q}$

This Newton map defines a $k[t]$-morphism $\pi_{q, \bar{j}}: \mathcal{L}(Y) \rightarrow \mathcal{L}(X)$ (in fact nothing is done on the variable $t)$. We can apply to $\pi_{q, \bar{j}}$ the change of variables formula, see theorem 2.2.

For $l=1, \ldots, d$, the $l$-th component of $\boldsymbol{\varphi}$ is $\varphi_{l}(t)=t^{\left(r_{l}+1\right) p_{l}^{q}} v_{l}(t)$, where $v_{l}(t) \in$ $k[[t]]$ such that $v_{l}(0)=\left(s_{l}^{0}\right)^{p_{l}^{q}}$. There exists a unique $w_{l}(t) \in k[[t]]$ such that $w_{l}(0)=$ $s_{l}^{0}$ and $w_{l}(t)^{p_{l}^{q}}=v_{l}(t)$. If $\psi_{l}(t):=t^{\left(r_{l}+1\right)} w_{l}(t)$ then

$$
\varphi_{d+1}(t)=t^{\left(r_{1}+1\right) \bar{b}_{1}^{q}+\ldots+\left(r_{d}+1\right) \bar{b}_{d}^{q}} v_{d+1}(t) \text { and } v_{d+1}(0)=\prod_{l=1}^{d}\left(s_{l}^{0}\right)^{\bar{b}_{l}^{q}} .
$$

The equation

$$
\begin{aligned}
t^{\left(r_{1}+1\right) \bar{b}_{1}^{q}+\ldots+\left(r_{d}+1\right) \bar{b}_{d}^{q}} v_{d+1}(t) & =\left(\psi_{d+1}(t)+\bar{\beta}_{\bar{j}}^{q}\right) \psi_{1}(t)^{\bar{b}_{1}^{q}} \ldots \psi_{d}(t)^{\bar{b}_{d}^{q}}= \\
& =\left(\psi_{d+1}(t)+\bar{\beta}_{\bar{j}}^{q}\right) t^{\left(r_{1}+1\right) \bar{b}_{1}^{q}+\ldots+\left(r_{d}+1\right) \bar{b}_{d}^{q}} w_{1}(t)^{\bar{b}_{1}^{q}} \ldots w_{d}(t)^{\bar{b}_{d}^{q}}
\end{aligned}
$$

completely determines an arc $\psi_{d+1}(t)$ such that $\psi_{d+1}(0)=0$. So there is a unique $\boldsymbol{\psi}:=\left(\psi_{1}, \ldots, \psi_{d}, \psi_{d+1}\right) \in \mathcal{L}_{0}(Y)$ such that $\pi_{q, \bar{j}}(\boldsymbol{\psi})=\boldsymbol{\varphi}$.

Moreover, if $\bar{h}_{q, \bar{j}}:=h \circ \pi_{q, \bar{j}}$ and $\omega_{q, \bar{j}}:=\bar{\omega} \circ \pi_{q, \bar{j}}$, we denote by $B_{n, m_{\mathbf{k}}}^{\mathbf{r}}$ the set of arcs $\boldsymbol{\psi} \in \mathcal{L}_{0}(Y)$ such that

- $\operatorname{ord}\left(\bar{h}_{q, \bar{j}} \circ \boldsymbol{\psi}\right)=n$,

- $\operatorname{ord}\left(\omega_{q, \bar{j}} \circ \boldsymbol{\psi}\right)=m_{\mathbf{k}}$,

- if $\mathbf{k}(\boldsymbol{\psi})=\left(\tilde{k}_{1}, \ldots, \tilde{k}_{d}, \tilde{k}_{d+1}\right)$ then $\tilde{k}_{l}=r_{l}+1$, for $l=1, \ldots, d$. 
Then $\pi_{q, \bar{j}}$ defines a bijection between $B_{n, m_{\mathbf{k}}}^{\mathbf{r}}$ and $V_{n, B, m_{\mathbf{k}}}^{\bar{\gamma}_{q}, \mathbf{g}^{1}, \bar{j}, \mathbf{r}}$. The order of the Jacobian of the $k[t]$-morphism $\pi_{q, \bar{j}}$ is constant on $B_{n, m_{\mathbf{k}}}^{\mathbf{r}}$ and equals to:

$$
\operatorname{ord}_{t}\left(\mathcal{J}_{\pi_{q, \bar{j}}}\right)=\sum_{l=1}^{d}\left(r_{l}+1\right)\left(p_{l}^{q}+\bar{b}_{l}^{q}-1\right)
$$

The set $B_{n, m_{\mathbf{k}}}^{\mathbf{r}}$ is strongly measurable in $\mathcal{L}(Y)$. Hence $V_{n, B, m_{\mathbf{k}}}^{\bar{\gamma}_{q}, \mathbf{g}^{1}, \bar{j}, \mathbf{r}}$, its bijective image by $\pi_{q, \bar{j}}$, is also strongly measurable in $\mathcal{L}(X)$, [16, Theorem A.8]. To measure $V_{n, B, m_{\mathbf{k}}}^{\bar{\gamma}_{q}, \mathbf{g}^{1}, \bar{j}, \mathbf{r}}$ the change variables formula gives

$$
\mu_{X}\left(V_{n, B, m_{\mathbf{k}}}^{\bar{\gamma}_{q}, \mathbf{g}^{1}, \bar{j}, \mathbf{r}}\right)=\int_{V_{n, B, m_{\mathbf{k}}}^{\bar{\gamma} q, \mathbf{g}^{1}, \bar{j}, \mathbf{r}}} d \mu_{X}=\int_{B_{n, m_{\mathbf{k}}}^{\mathbf{r}}} \mathbb{L}^{-\operatorname{ord}_{t} \mathcal{J}_{\pi_{q, \bar{j}}(y)}} d \mu_{Y} .
$$

If $\widehat{\omega}_{1}$ is the pullback by $\pi_{q, \bar{j}}$ of the differential form $d \bar{x}_{1} \wedge \cdots \wedge d \bar{x}_{d} \wedge d \bar{z}$ then $\mu_{X}\left(V_{n, B, m_{\mathbf{k}}}^{\bar{\gamma}_{q}, \mathbf{g}^{1}, \bar{j}, \mathbf{r}}\right)=\sum_{k \in \mathbb{P}} \mathbb{L}^{-k} \mu_{Y}\left(B_{n, m_{\mathbf{k}}}^{\mathbf{r}} \cap\left\{\operatorname{ord}_{t}\left(\widehat{\omega}_{1}\right)=k\right\}\right)=\mathbb{L}^{-\sum_{l=1}^{d}\left(r_{l}+1\right)\left(p_{l}^{q}+\bar{b}_{l}^{q}-1\right)} \mu_{Y}\left(B_{n, m_{\mathbf{k}}}^{\mathbf{r}}\right)$.

Putting all these terms together

$$
Z^{\bar{\gamma}_{q}, \mathbf{g}^{1}, \bar{j}, \mathbf{r}}(T)=\sum_{n \geq 1} \mathbb{L}^{-\sum_{l=1}^{d}\left(r_{l}+1\right)\left(p_{l}^{q} \nu_{l}+\bar{b}_{l}^{q}-1\right)} \mu_{Y}\left(B_{n, m_{\mathbf{k}}}^{\mathbf{r}}\right) T^{n} .
$$

Since $\operatorname{ord}_{t}\left(\omega_{q, \bar{j}} \circ \boldsymbol{\psi}\right)=\sum_{l=1}^{d}\left(r_{l}+1\right)\left(p_{l}^{q} \nu_{l}+\bar{b}_{l}^{q}-1\right)$ for any arc $\boldsymbol{\psi} \in B_{n, m}^{r}$,

$$
Z^{\bar{\gamma}_{q}, \mathbf{g}^{1}, \bar{j}, \mathbf{r}}(T)=\sum_{n \geq 1} \mathbb{L}^{-m_{\mathbf{k}}} \mu_{Y}\left(B_{n, m_{\mathbf{k}}}^{\mathbf{r}}\right) T^{n} .
$$

The set $B_{n, m}^{\mathbf{r}}$ is empty if $m \neq m_{\mathbf{k}}$ so

$$
Z^{\bar{\gamma}_{q}, \mathbf{g}^{1}, \bar{j}}(T)=\sum_{\mathbf{r} \in \mathbb{N}^{d}} \sum_{n \geq 1} \mathbb{L}^{-m_{\mathbf{k}}} \mu_{Y}\left(B_{n, m_{\mathbf{k}}}^{\mathbf{r}}\right) T^{n}=Z_{D L}\left(\bar{h}_{q, \bar{j}}, \omega_{q, \bar{j}}, T\right), \forall \bar{j}=1, \ldots, v(q) .
$$

Remark 5.24. If $h$ has $\mathbf{e v}(h)=1$, no more steps to get a formula for $Z_{D L}(h, \omega, T)$ are needed, see section 6.2. This happen because each fundamental set $G^{q}$ has only one element.

Step 9. Computations for $h^{t}, \mathbf{g} \in G^{q, \alpha_{q, j}}$ and $w=1, \ldots, w(q, j)$.

By the proof of Lemma 5.14, in particular its definition in (5.10) one can write $\mathbf{g}=\left(\mathbf{0}, \frac{\alpha_{q, j}}{n_{1}^{q}}\right)+\sum_{l=1}^{d} \mu_{l}^{\mathbf{g}} \mathbf{w}_{l}^{q}$. Thus

$$
\mathbf{g}=\left(\mu_{1}^{\mathbf{g}} p_{1}^{q}, \ldots, \mu_{d}^{\mathbf{g}} p_{d}^{q}, \frac{\alpha_{q}}{n_{1}^{q}}+\sum_{l=1}^{d} \mu_{l}^{\mathbf{g}} \bar{b}_{l}^{q}\right) \in \mathbb{P}^{d+1} .
$$

By definition of $G^{q, \alpha_{q, j}}, 0<\mu_{l}^{\mathrm{g}} \leq 1$ is rational. Choose $v_{1}, \ldots, v_{d} \in \mathbb{P}$ such that $\mu_{l}^{\mathbf{g}}=\frac{v_{l}}{p_{l}^{q}}, l=1, \ldots, d$ and let $c^{\mathbf{g}} \in \mathbb{P}$ the $(d+1)^{\mathrm{th}}$-coordinate of $\mathbf{g}$. 
Fix $\mathbf{r} \in \mathbb{N}^{d}$. Let $\varphi \in V_{n, B, m}^{t, q, j, \mathbf{g}, w, \mathbf{r}}=\left\{\boldsymbol{\varphi} \in V_{n, B, m}^{t, q, j, \mathbf{g}}: \mathbf{k}(\boldsymbol{\varphi})=\mathbf{g}+r_{1} \mathbf{w}_{1}+\ldots+r_{d} \mathbf{w}_{d}\right\}$ be an arc and denote:

- $\mathbf{k}:=\mathbf{k}(\boldsymbol{\varphi})=\left(\left(\mu_{1}^{\mathbf{g}}+r_{1}\right) p_{1}^{q}, \ldots,\left(\mu_{d}^{\mathbf{g}}+r_{d}\right) p_{d}^{q}, \frac{\alpha_{q, j}}{n_{1}^{q}}+\sum_{l=1}^{d}\left(\mu_{l}^{\mathbf{g}}+r_{l}\right) \bar{b}_{l}^{q}\right)$.

- $\mathbf{a}:=\mathbf{a}(\boldsymbol{\varphi}) \in \mathbf{G}_{m, k}^{d+1} \cap\left\{\widehat{f}_{q, j}^{t, w}=0\right\}$, where $\widehat{f}_{q, j}^{t, w}=z^{n_{1}^{q}}-\beta_{w} \mathbf{x}_{1}^{n_{1}^{q} \boldsymbol{\lambda}_{\kappa_{q}}}$. Consider $\mathbf{a}=\pi_{j}^{q, w}\left(s^{0}\right), s^{0} \in \mathbf{G}_{m, k}^{d}$ with the fixed parametrization of $\left\{\hat{f}_{q, j}^{t, w}=0\right\}$ induced by $\pi_{j}^{q, w}: \mathbb{A}_{k}^{d} \rightarrow \mathbf{G}_{m, k}^{d+1} \cap\left\{\widehat{f}_{q, j}^{t, w}=0\right\}:\left(s_{1}, \ldots, s_{d}\right) \mapsto\left(s_{1}^{p_{1}^{q}}, \ldots, s_{d}^{p_{d}^{q}}, \alpha_{w} s_{1}^{\bar{b}_{1}^{q}} \ldots s_{d}^{\bar{b}_{d}^{q}}\right)$ where $\left(\alpha_{w}\right)^{n_{1}^{q}}=\beta_{w}$.

For each $\boldsymbol{\varphi}$ with $\mathbf{k}(\boldsymbol{\varphi})=\mathbf{k}$ then $m_{\mathbf{k}}:=\operatorname{ord}(\omega \circ \boldsymbol{\varphi})=\sum_{l=1}^{d}\left(r_{l}+\mu_{l}^{\mathbf{g}}\right) p_{l}^{q}\left(\nu_{l}-1\right)$. Since $m_{\mathbf{k}}$ is the unique value of $m$ for which $V_{n, B, m}^{t, q, j, \mathbf{g}, w, \mathbf{r}}$ may be non-empty,

$$
Z_{q, j}^{\mathcal{W}, \mathbf{g}, w}(T)=\sum_{r \in \mathbb{N}^{d}} Z_{q, j}^{\mathcal{W}, \mathbf{g}, w, \mathbf{r}}(T)=\sum_{r \in \mathbb{N}^{d}} \mathbb{L}^{-m_{\mathbf{k}}} \mu_{X}\left(V_{n, B, m_{\mathbf{k}}}^{t, q, j, \mathbf{g}, \mathbf{r}}\right) T^{n} .
$$

Let $Y=\mathbb{A}_{k}^{d+1}$ and let $\pi_{\mathbf{g}}: \mathcal{L}(Y) \rightarrow \mathcal{L}(X)$ be the $k[t]$-morphism defined by $x_{l}=t^{v_{l}} y_{l}$, for every $l=1, \ldots, d$, and $z=t^{c^{\mathbf{g}}} z_{1}$.

Lemma 5.25. Under the above conditions:

(1) The map $\pi_{\mathbf{g}}$ defines a $k[t]$-morphism.

(2) The Jacobian of $\pi_{\mathbf{g}}$ has constant order $\nu_{\mathbf{g}}:=\operatorname{ord}_{t}\left(J_{\pi_{\mathbf{g}}}\right)=\sum_{l=1}^{d} v_{l} c^{\mathbf{g}}$ on the arcs of $\mathcal{L}(Y)$.

(3) The $K$-quasi-ordinary function $h^{\{t\}}:=h^{t} \circ \pi_{\mathrm{g}}$ has the same characteristic exponents (and monomials) as $h^{t}$, and consequently the same as $h$ (this follows directly from Remark 4.30).

Fix $\mathbf{r} \in \mathbb{N}^{d}$, and take an arc $\varphi \in V_{n, B, m_{\mathrm{k}}}^{t, q, j, \mathbf{g}, w, \mathbf{r}}$. There exists a unique arc $\boldsymbol{\psi} \in \mathcal{L}(Y)$ such that $\boldsymbol{\varphi}=\pi_{\mathrm{g}} \circ \boldsymbol{\psi}$. Properties of $\boldsymbol{\psi}$ will depend on $\mathbf{r}$ :

(2.a) $\mathbf{r} \in \mathbb{P}^{d}$; in this case $\boldsymbol{\psi} \in \mathcal{L}_{0}(Y), \mathbf{a}(\boldsymbol{\psi})=\mathbf{a}$ and

$$
\mathbf{k}(\psi)=\left(r_{1} p_{1}^{q}, \ldots, r_{d} p_{d}^{q}, \sum_{l=1}^{d} r_{l} \bar{b}_{l}^{q}\right) .
$$

(2.b) $\mathbf{r} \in \mathbb{N}^{d} \backslash \mathbb{P}^{d}$; in this case $\boldsymbol{\psi} \in \mathcal{L}_{(\mathbf{y}, 0)}(Y)$. More precisely; let $J_{\mathbf{r}} \varsubsetneqq\{1, \ldots, d\}$ be the set of indices such that $r_{l}=0 \Leftrightarrow l \in J_{\mathbf{r}}$; we observe that $\emptyset \neq J_{\mathbf{r}} \varsubsetneqq$ $\{1, \ldots, d\}$. The point $\mathbf{y}$ corresponds to $\left(y_{1}, \ldots, y_{d}\right)$ where $y_{l}=\left(s_{l}^{0}\right)_{l}^{q}$ if $l \in J_{\mathbf{r}}$ and $y_{l}=0$ otherwise. We include in this case the case where $\mathbf{r}=\mathbf{0} \in \mathbb{N}^{d}$ and $J=\{1, \ldots, d\}$.

Remark 5.26. Note that in fact $J \subset J_{q}$, see Remark 5.23.

$$
\begin{gathered}
\text { Decompose } Z_{q, j}^{\mathcal{W}, \mathbf{g}, w}(T)=Z_{1, q, j}^{\mathcal{W}, \mathbf{g}, w}(T)+\sum_{\emptyset \varsubsetneqq J \subset\{1, \ldots, d\}} Z_{J, q, j}^{\mathcal{W}, \mathbf{g}, w}(T), \text { where } \\
Z_{1, q, j}^{\mathcal{W}, \mathbf{g}, w}(T):=\sum_{\mathbf{r} \in \mathbb{P}^{d}} \sum_{n \geq 1} \mathbb{L}^{-m_{\mathbf{k}}} \mu_{X}\left(V_{n, B, m_{\mathbf{k}}}^{t, q, \mathbf{g}, w, \mathbf{r}}\right) T^{n}
\end{gathered}
$$


and

$$
Z_{J, q, j}^{\mathcal{W}, \mathbf{g}, w}(T):=\sum_{\mathbf{r} \in \mathbb{N}^{d}: J=J_{\mathbf{r}}} \sum_{n \geq 1} \mathbb{L}^{-m_{\mathbf{k}}} \mu_{X}\left(V_{n, B, m_{\mathbf{k}}}^{t, q, j, \mathbf{g}, w, \mathbf{r}}\right) T^{n}
$$

Define $\omega_{\mathbf{g}}^{\{t\}}:=\pi_{\mathbf{g}}^{*} \omega$ and for $\mathbf{r} \in \mathbb{P}^{d}$ denote by $W_{n, B, m_{\mathbf{k}}}^{t, q, j, \mathbf{g}, \mathbf{r}}$ the semialgebraic subset of $\operatorname{arcs} \boldsymbol{\psi} \in \mathcal{L}_{0}(Y)$ such that:

- $\operatorname{ord}\left(h^{\{t\}} \circ \boldsymbol{\psi}\right)=n$.

- $\mathbf{k}(\boldsymbol{\psi})=\left(r_{1} p_{1}^{q}, \ldots, r_{d} p_{d}^{q}, \sum_{l=1}^{d} r_{l} \bar{b}_{l}^{q}\right)$.

- $\operatorname{ord}\left(\omega_{\mathbf{g}}^{\{t\}} \circ \boldsymbol{\psi}\right)=m_{\mathbf{k}}+\nu_{\mathbf{g}}$. The differential form $\omega_{\mathbf{g}}^{\{t\}}$ is defined as $t^{A_{\mathbf{g}}} \omega_{\mathbf{g}}$ where $\omega_{\mathbf{g}}:=\prod_{l=1}^{d} y_{l}^{\nu_{l}-1} d \mathbf{y} \wedge d z_{1}$ and $A_{\mathbf{g}}:=\sum_{l=1}^{d} v_{l}\left(\nu_{l}-1\right)$. Then $\tilde{m}_{\mathbf{k}}:=$ $\operatorname{ord}\left(\omega_{\mathbf{g}} \circ \boldsymbol{\psi}\right)=m_{\mathbf{k}}-A_{\mathbf{g}}$ and

$$
\nu_{\mathbf{g}}+A_{\mathbf{g}}=\frac{\alpha_{q}}{n_{1}^{q}}+\sum_{l=1}^{d} \mu_{l}^{\mathbf{g}}\left(p_{l}^{q} \nu_{l}+\bar{b}_{l}^{q}\right) .
$$

The map $\pi_{\mathbf{g}}$ induces a bijection between the sets $V_{n, B, m_{\mathbf{k}}}^{t, q, j, \mathbf{g}, w, \mathbf{r}}$ and $W_{n, B, m_{\mathbf{k}}}^{t, q, j, \mathbf{g}, w, \mathbf{r}}$. Since $\mu_{X}\left(V_{n, B, m_{\mathbf{k}}}^{t, q, j, \mathbf{g}, w, \mathbf{r}}\right)=\mathbb{L}^{-\nu_{\mathbf{g}}} \mu_{Y}\left(W_{n, B, m_{\mathbf{k}}}^{t, q, j, \mathbf{g}, w, \mathbf{r}}\right)$, applying the change of variables formula to $\pi_{\mathrm{g}}$ we have

$$
\begin{aligned}
Z_{1, q, j}^{\mathcal{W}, \mathbf{g}, w}(T) & =\sum_{\mathbf{r} \in \mathbb{P}^{d}} \sum_{n \geq 1} \mathbb{L}^{-m_{\mathbf{k}}-\nu_{\mathbf{g}}} \mu_{Y}\left(W_{n, B, m_{\mathbf{k}}}^{t, q, j, \mathbf{g}, w, \mathbf{r}}\right) T^{n} \\
& =\sum_{\mathbf{r} \in \mathbb{P}^{d}} \sum_{n \geq 1} \mathbb{L}^{-\nu_{\mathbf{g}}-A_{\mathbf{g}}-\operatorname{ord}_{t}\left(\omega_{\mathbf{g}}\right)} \mu_{Y}\left(W_{n, B, m_{\mathbf{k}}}^{t, q, j, \mathbf{g}, w, \mathbf{r}}\right) T^{n} \\
& =\mathbb{L}^{-\left(\nu_{\mathbf{g}}+A_{\mathbf{g}}\right)} \sum_{\mathbf{r} \in \mathbb{P}^{d}} \sum_{n \geq 1} \mathbb{L}^{-\tilde{m}_{\mathbf{k}}} \mu_{Y}\left(W_{n, B, \tilde{m}_{\mathbf{k}}}^{t, q, j, \mathbf{g}^{1}, w, \mathbf{r}}\right) T^{n}
\end{aligned}
$$

Consider the following Newton map associated with the Newton component of $h^{t}$ (as a $K$-quasi-ordinary power series) we are dealing with. Its Newton polyhedron is defined by $z^{n_{1}^{q}}-\boldsymbol{\beta}_{j, w}^{q}(t) x_{1}^{b_{1}^{q}} \ldots x_{d}^{b_{d}^{q}}$, where $\boldsymbol{\beta}_{j, w}^{q}(t)=\beta_{w} t^{\alpha_{q, j}}+$ higher order terms $\epsilon$ $k[[t]]$ and $\beta_{w} \in \mathbf{G}_{m, k}$. We can write $\boldsymbol{\beta}_{j, w}^{q}(t)=t^{\alpha_{q, j}} \alpha(t)$ being $\alpha(t)=\left(\alpha_{w}+\ldots\right)^{n_{1}^{q}} \in$ $k[[t]]$ because $\left(\alpha_{w}\right)^{n_{1}^{q}}=\beta_{w}$.

Let $\pi_{j, t}^{q, w}$ be the $k[[t]]$-morphism associated to the transformation $y_{l}=\tilde{y}_{l}^{q}$ for all $i=1, \ldots, d$ and $z_{1}=\left(z_{2}+\alpha(t)\right) \prod_{l=1}^{d} \tilde{y}_{l}^{q}$. Recall the change of variables formula is also applied here, see Remark 2.4.

Taking into account the pull-back of the discriminants and applying the same proof as in Step 8, the following proposition follows.

Proposition 5.27. Let $h_{q, j, \mathbf{g}, w}^{t}:=h^{\{t\}} \circ \pi_{j, t}^{q, w}=h^{t} \circ \pi_{\mathbf{g}} \circ \pi_{j, t}^{q, w}$ and $\tilde{\omega}_{q, j, \mathbf{g}, w}=\left(\pi_{j, t}^{q, w}\right)^{*} \omega_{\mathbf{g}}$. Then: 
(1) The series $h_{q, j, \mathbf{g}, w}^{t}$ is $\mathcal{W}$-quasi-ordinary and its depth is strictly less than the one of $h^{t}$. We have

$$
Z_{1, q, j}^{\mathcal{W}, \mathbf{g}, w}(T)=\mathbb{L}^{-\left(\nu_{\mathbf{g}}+A_{\mathbf{g}}\right)} Z_{D L}^{\mathcal{W}}\left(h_{q, j, \mathbf{g}, w}^{t}, \tilde{\omega}_{q, j, \mathbf{g}, w}, T\right) .
$$

(2) If $h^{t}$ is $\widetilde{\mathcal{W}}$-quasi-ordinary and Newton compatible then there exists a weight $\widetilde{\mathcal{W}^{\prime}}$ such that $h_{q, j, \mathbf{g}, w}^{t}$ is $\widetilde{\mathcal{W}^{\prime}}$-quasi-ordinary and Newton compatible.

(3) The series $h_{q, j, \mathbf{g}, w}^{t}$ is $J_{q}$-bounded, see Remark 5.23.

Proof. The only non trivial facts are the two last ones. It is easy to prove from the Newton map that $h_{q, j, \mathbf{g}, w}^{t}$ is $\widetilde{\mathcal{W}^{\prime}}$-quasi-ordinary, where

$$
\widetilde{\mathcal{W}^{\prime}}:=\left(p n_{1}^{q} ;\left(p v_{1}+g_{1}\right) c_{1}^{q}, \ldots,\left(p v_{d}+g_{d}\right) c_{d}^{q}\right) .
$$

This is due to the following equalities:

$$
\alpha_{q, j}=\frac{1}{p} \sum_{l=1}^{d} b_{l}^{q} g_{l}, \quad c^{\mathbf{g}}=\frac{\alpha_{q, j}}{n_{1}^{q}}+\sum_{l=1}^{d} \frac{v_{l}}{p_{l}^{q}} \bar{b}_{l}^{q}=\frac{\alpha_{q, j}}{n_{1}^{q}}+\sum_{l=1}^{d} \frac{v_{l}}{n_{1}^{q}} b_{l}^{q}=\frac{1}{n_{1}^{q}} \sum_{l=1}^{d}\left(v_{l}+\frac{g_{l}}{p}\right) b_{l}^{q} .
$$

The $\widetilde{\mathcal{W}}{ }^{\prime}$ depends only on $\widetilde{\mathcal{W}}$ and on the Newton map. It implies that we can choose a generic $\widetilde{\mathcal{W}}$-quasi-ordinary series $\tilde{h}^{t}$ with the same characteristic monomials and then Newton compatible. Then, $\tilde{h}_{q, j, \mathbf{g}, w}^{t}$ is also $\widetilde{\mathcal{W}}^{\prime}$-quasi-ordinary. If we pass to good coordinates the weight $\widetilde{\mathcal{W}}^{\prime}$ remains and both power series have the same Newton polyhedron. For $\tilde{h}_{q, j, \mathbf{g}, w}^{t}$ all the coefficients in its restriction to the Newton polyhedron will be non zero and it is easily seen that it implies that $\tilde{h}_{q, j, \mathbf{g}, w}^{t}$ is Newton compatible and it is also the case for $h_{q, j, \mathbf{g}, w}^{t}$.

The fact that $h_{q, j, \mathbf{g}, w}^{t}$ is $J_{q}$-bounded follows from the behaviour of the $t$-morphism $\pi_{\mathbf{g}}$; note that each variable of $J_{q}$ is replaced by this variable times a power of $t$; then for a fixed power of $t$ only a finite number of monomials in $t$ and the variables of $J_{q}$ can contribute.

Consider now arcs in Case $(2 \underline{b})$. Let $\emptyset \subsetneq J \subset\{1, \ldots, d\}$; in fact, from Remark 5.26. we are only interested in the case $\emptyset \subsetneq J \subset J_{q}$. Let us fix a point $(\mathbf{y}, 0)$ corresponding to the subset $J$. Since $h_{q, j, \mathbf{g}, w}^{t}$ is $\mathcal{W}$-quasi-ordinary $J$-bounded power series the germ of $h_{q, j, \mathbf{g}, w}^{t}$ at $(\mathbf{y}, 0)$ is also a $\mathcal{W}$-quasi-ordinary power series, see Remark 5.2. We can indeed evaluate at $(\mathbf{y}, 0)$ because the coefficients of its monomials in $t$ belong to $k[\widetilde{\mathbf{y}}, z]$. Since $h_{q, j, \mathbf{g}, w}^{t}$ is $\mathcal{W}$-quasi-ordinary its discriminant is computed from the pull-back of $D_{z}\left(h^{t}\right)=t^{\beta} x_{1}^{\alpha_{1}} \ldots x_{d}^{\alpha_{d}} \varepsilon(\mathbf{x}, t)$ where $\varepsilon(0,0) \neq 0$. Since $\mathbf{g} \in \mathbb{P}^{d+1}$ then $\tilde{\varepsilon}:=\varepsilon \circ \pi_{\mathbf{g}} \circ \pi_{t, j}^{q, w}(\tilde{\mathbf{y}}, t)$ also verifies $\tilde{\varepsilon}(0,0) \neq 0$. The characteristic exponents do not depend on the particular $\mathbf{y}$ but only on $J$ and the depth has decreased. Namely, by induction $Z_{D L}^{\mathcal{W}}\left(\left(h_{q, j, \mathbf{g}, w}^{t}\right)_{(\mathbf{y}, 0)},\left(\tilde{\omega}_{q, j, \mathbf{g}, w}\right)_{(\mathbf{y}, 0)}, T\right)$ satisfies the theorem. Observe also that the variety of such $\mathbf{y}$ 's is $\mathbb{G}_{m, k}^{\# J}$.

Proposition 5.28. The following equality holds:

$$
Z_{J, q, j}^{\mathcal{W}, \mathbf{g}, w}(T)=(\mathbb{L}-1)^{\# J} \mathbb{L}^{-\left(\nu_{\mathbf{g}}+A_{\mathbf{g}}\right)} Z_{D L}^{\mathcal{W}}\left(\left(h_{q, j, \mathbf{g}, w}^{t}\right)_{(\mathbf{y}, 0)},\left(\tilde{\omega}_{q, j, \mathbf{g}, w}\right)_{(\mathbf{y}, 0)}, T\right),
$$

where $(\mathbf{y}, 0)$ means the germ at this point. 
Proof. This result is a consequence of Proposition 5.34.

Remark 5.29. We note that $\left(h_{q, j, \mathbf{g}, w}^{t}\right)_{(\mathbf{y}, 0)}$ is a $\mathcal{W}$-quasi-ordinary series in good coordinates but its depth is strictly less than $\operatorname{depth}\left(h_{q, j, \mathbf{g}, w}^{t}\right)$. Moreover its Newton polyhedron is obtained projecting the Newton polyhedron of $h_{q, j, \mathbf{g}, w}^{t}$ on the corresponding coordinate planes.

Step 10. Computations for $h$ and $\mathbf{g}=\mu_{1}^{\mathbf{g}} \mathbf{w}_{1}^{q}+\ldots+\mu_{d}^{\mathbf{g}} \mathbf{w}_{d}^{q} \in G^{q}$ such that $0<\mu_{l}^{\mathbf{g}}<1$ for some $l \in\{1, \ldots, d\}$.

This case can be computed as in step 9 since we may consider $h$ as $\widetilde{\mathcal{W}}$-quasiordinary where $\widetilde{\mathcal{W}}=(1 ; 0, \ldots, 0)$.

We are going to collect all the results in this section to compare motivic zeta functions for the functions $h^{t}$ and $h$ in order to obtain recurrence formulæ.

By the identities in (5.13),

$$
\begin{aligned}
Z_{D L}^{\mathcal{W}}\left(h^{t}, \omega, T\right) & =Z_{A}^{\mathcal{W}}\left(h^{t}, \omega, T\right)+ \\
& +\sum_{q=1}^{r} \sum_{\substack{j=1 \\
s_{1}^{j, q}=1}}^{u(q)}\left(\sum _ { \mathbf { g } \in G ^ { q , \alpha _ { q , j } } } \mathbb { L } ^ { - ( \nu _ { \mathbf { g } } + A _ { \mathbf { g } } ) } \left(\sum _ { w = 1 } ^ { w ( q , j ) } \left(Z_{D L}^{\mathcal{W}}\left(h_{q, j, \mathbf{g}, w}^{t}, \tilde{\omega}_{q, j, \mathbf{g}, w}^{t}, T\right)+\right.\right.\right. \\
& \left.\left.+\sum_{\emptyset \subseteq J \subset\{1, \ldots, d\}}(\mathbb{L}-1)^{\# J} Z_{D L}^{\mathcal{W}}\left(\left(h_{q, j, \mathbf{g}, w}^{t}\right)_{(\mathbf{y}, 0)},\left(\tilde{\omega}_{q, j, \mathbf{g}, w}\right)_{(\mathbf{y}, 0)}, T\right)\right)\right)
\end{aligned}
$$

where $\nu_{\mathbf{g}}+A_{\mathbf{g}}=\frac{\alpha_{q, j}}{n_{1}^{q}}+\sum_{l=1}^{d} \mu_{l}^{\mathbf{g}}\left(p_{l}^{q} \nu_{l}+\bar{b}_{l}^{q}\right)$. In the same way,

$$
\begin{aligned}
Z_{D L}(h, \bar{\omega}, T) & =Z_{D L}^{A}(h, \bar{\omega}, T)+\sum_{q=1}^{r} \sum_{\bar{j}=1}^{v(q)}\left(Z_{D L}\left(\bar{h}_{q, \bar{j}}, \bar{\omega}_{q, \bar{j}}, T\right)\right. \\
& +\sum_{\mathbf{g} \in G^{q}, \mathbf{g} \neq \mathbf{g}^{1}}\left(\mathbb { L } ^ { - ( \nu _ { \mathbf { g } } + A _ { \mathbf { g } } ) } \left(Z_{D L}^{\mathcal{W}}\left(\tilde{h}_{q, \mathbf{g}, \bar{j}}^{t}, \tilde{\omega}_{q, \mathbf{g}, \bar{j}}, T\right)+\right.\right. \\
& \left.\left.+\sum_{\emptyset \subseteq J \subset\{1, \ldots, d\}}(\mathbb{L}-1)^{\# J} Z_{D L}^{\mathcal{W}}\left(\left(\tilde{h}_{q, \mathbf{g}, \bar{j}}^{t}\right)_{(\mathbf{y}, 0)},\left(\tilde{\omega}_{q, \mathbf{g}, \bar{j}}\right)_{(\mathbf{y}, 0)}, T\right)\right)\right) .
\end{aligned}
$$

where $v(q)=\sum_{j=1}^{u(q)} w(q, j)$ and $\nu_{\mathbf{g}}+A_{\mathbf{g}}=\sum_{l=1}^{d} \mu_{l}^{\mathbf{g}}\left(p_{l}^{q} \nu_{l}+\bar{b}_{l}^{q}\right)$.

Proof of Theorem 5.3. The starting point of the induction has been done in Step 1,

Let us attack the general case. Since $h^{t}$ and $h$ (as $K$-quasi-ordinary power series) have the same characteristic exponents then by Lemma 4.29 there exist one-to-one correspondences between the sets of faces of their Newton polyhedra and their sets of roots on such faces for $h^{t}$ and $h$. Assume that under these bijections the $\operatorname{root} \boldsymbol{\beta}_{j, w}^{q}(t)$, where $s_{1}^{q, j}=1$, corresponds to $\bar{\beta}_{\bar{j}}^{q}$, namely the polynomial $z^{n_{1}^{q}}-\boldsymbol{\beta}_{j, w}^{q}(t) x_{1}^{b_{1}^{q}} \ldots x_{d}^{b_{d}^{q}}$ 
corresponds to $\bar{z}^{n_{1}^{q}}-\bar{\beta}_{\bar{j}}^{q} \bar{x}_{1}^{b_{1}^{q}} \ldots \bar{x}_{d}^{b_{d}^{q}}$. Let $h_{\boldsymbol{\beta}}^{t}$ and $\bar{h}_{q, \bar{j}}$ denote the pull-back of $h^{t}$ and $h$ under the corresponding Newton maps associated with the roots $\boldsymbol{\beta}_{j, w}^{q}(t)$ and $\bar{\beta}_{\bar{j}}^{q}$.

(1) By Lemma 4.29, the characteristic exponents of $h_{\boldsymbol{\beta}}^{t}$ and $\bar{h}_{q, \bar{j}}$ are equal.

(2) For each $\mathbf{g} \in G^{q}$ (resp. $\mathbf{g} \in G^{q, \alpha_{q, j}}$ ) the $K$-quasi-ordinary power series $h \circ \pi_{\mathbf{g}}$ (resp. $h^{t} \circ \pi_{\mathbf{g}}$ ) has the same characteristic exponents as $h\left(\right.$ resp. $\left.h^{t}\right)$. This is Remark 4.30.

(3) Applying (1) and (2) for each $\mathbf{g} \in G^{q} \backslash\left\{\mathbf{g}^{1}\right\}$, then $\tilde{h}_{q, \mathbf{g}, \bar{j}}$ has the same characteristic exponents as $\bar{h}_{q, \bar{j}}$ (recall that $\bar{h}_{q, \bar{j}}$ is obtained in Step 8 ).

(4) In the same way, for each $\mathbf{g} \in G^{q, \alpha_{q, j}}, h_{q, j, \mathbf{g}, w}^{t}$ has the same characteristic exponents as $h_{\boldsymbol{\beta}}^{t}$ which has the same as $\bar{h}_{q, \bar{j}}$. In all these cases, the depth of all these quasi-ordinary power series are smaller than depth $(h)$. In particular we can follow by induction.

(5) For the statement of the Theorem the terms $\mathbb{L}^{-\nu}$ are not essential then we have that

$$
Z_{D L}^{\mathcal{W}}\left(\tilde{h}_{q, \mathbf{g}, \bar{j}}^{t}, \tilde{\omega}_{q, \mathbf{g}, \bar{j}}, T\right) \in \mathbb{Z}\left[\mathbb{L}, \mathbb{L}^{-1},\left(1-\mathbb{L}^{-\nu} T^{N}\right)^{-1}\right][T],
$$

where $(N, \nu) \in C P\left(\bar{h}_{q, \bar{j}}, \bar{\omega}_{q, \bar{j}}\right)$.

Proof of Theorem 5.9. The starting point of the induction was done in Step 1 .

For the general case we consider the formulæ (5.18) and (5.19). In this case the restriction $s_{1}^{j, q}=1$ is empty and the series appearing in the second line of (5.18) are $\widetilde{\mathcal{W}}^{\prime}$-quasi-ordinaries for some weights and they have depth less than $h$. The series in the third line are only $\mathcal{W}$-quasi-ordinaries.

Because of Theorem 5.3 we can compute $\chi_{\text {top }}\left(\bullet,\left(\mathbb{L}^{-s}\right)\right)$ for each term. For the second line terms we deduce by induction that

$$
\chi_{\text {top }}\left(\mathbb{L}^{-\left(\nu_{\mathbf{g}}+A_{\mathbf{g}}\right)} Z_{D L}^{\mathcal{W}}\left(h_{q, j, \mathbf{g}, w}^{t}, \tilde{\omega}_{q, j, \mathbf{g}, w},\left(\mathbb{L}^{-s}\right)\right)\right)=Z_{\text {top }, 0}\left(\bar{h}_{q, \bar{j}}, \omega_{q, \bar{j}}, s\right) .
$$

By Theorem [5.3 and (5.17), the terms in the third line vanish when applying $\chi_{\text {top }}\left(\bullet\left(\mathbb{L}^{-s}\right)\right)$.

\subsection{Zeta functions along strata.}

To finish the proof of Theorems 5.3 and 5.9 we must prove Proposition 5.17. We will proceed by induction in families of $\mathcal{W}$-quasi-ordinary power series.

Definition 5.30. Let $h^{t} \in k[[t]][[\mathbf{x}, \mathbf{y}]][z]$ be a $\mathcal{W}$-quasi-ordinary power series, $\mathbf{x}=$ $\left(x_{1}, \ldots, x_{d}\right), \mathbf{y}=\left(y_{1}, \ldots, y_{e}\right)$ which is bounded for the $\mathbf{y}$-variables. We say that $h^{t}$ is a family of $\mathcal{W}$-quasi-ordinary power series if for any $\mathbf{y}^{0} \in \mathbf{G}_{k, m}^{e}$, the well-defined germ $h_{\mathbf{y}^{0}}^{t}:=h^{t}\left(\mathbf{x}, \mathbf{y}+\mathbf{y}^{0}\right)$ is also $\mathcal{W}$-quasi-ordinary power series with the same characteristic monomials. 
Definition 5.31. The Denef-Loeser zeta function of a family of $\mathcal{W}$-quasi-ordinary power series and a form $\omega$ satisfying the support condition (2.3) is defined by

$$
\begin{array}{r}
Z_{D L}^{\mathcal{W}, \mathbf{y}}\left(h^{t}, \omega, T\right):=T^{\theta} \sum_{n \in \mathbb{N}}\left(\sum_{m \in \mathbb{N}} \mathbb{L}^{-m} \mu_{X}\left(V_{n, m}^{t}\right)\right) T^{n} \in \widehat{\mathcal{M}}_{k}[[T]], \\
Z_{\mathrm{top}, 0}^{\mathcal{W}}\left(h^{t}, \omega, s\right):=\chi_{\mathrm{top}}\left(Z_{D L}^{\mathcal{W}}\left(h^{t}, \omega, \mathbb{L}^{-s}\right)\right) .
\end{array}
$$

where we are considering arcs having their origin along $\{0\} \times \mathbf{G}_{k, m}^{e}$.

Remark 5.32. The powers of the variables in $\mathbf{y}$ for $\omega$ are negligible.

Lemma 5.33. $Z_{D L}^{\mathcal{W}, \mathbf{y}}\left(h^{t}, \omega, T\right)=Z_{D L}^{\mathcal{W}, \mathbf{y}}\left(\tilde{h}^{t}, \omega, T\right)$ where $\tilde{h}^{t}(\mathbf{x}, \mathbf{y})=h^{t}\left(\mathbf{x}, \mathbf{y}^{\mathbf{n}}\right)$.

Proposition 5.34. Let $h^{t}$ be a family of $\mathcal{W}$-quasi-ordinary power series and $\omega$ a form verifying the support condition (2.3). Then for all $\mathbf{y}^{0} \in \mathbf{G}_{k, m}^{e}$,

$$
Z_{D L}^{\mathcal{W}, \mathbf{y}}\left(h^{t}, \omega, T\right)=(\mathbb{L}-1)^{e} Z_{D L}^{\mathcal{W}}\left(h_{\mathbf{y}^{0}}^{t}, \omega, T\right) .
$$

Proof. The proof is by induction on the depth of $h^{t}$; if depth $h^{t}=0$, the result is trivial. We compute $Z_{D L}^{\mathcal{W}, \mathbf{y}}\left(h^{t}, \omega, T\right)$ following the Newton polyhedron and the Newton maps.

We begin with the $A$-part. If we consider a vertex, it is related to a monomial $t^{\alpha} \mathbf{x}^{\mathbf{n}} \mathbf{y}^{\mathbf{m}} z^{n}$. It is clear that both terms in the equality differ by an $(\mathbb{L}-1)^{e}$ factor. Let us fix an edge of the Newton polytope and a factor of the principal part which will give the key for the Newton map, i.e., we fix some factor $\left(z^{n}-\beta t^{\alpha} \mathbf{x}^{\mathbf{n}} \mathbf{y}^{\mathbf{m}}\right)^{m}$.

The mapping

$$
(\mathbf{x}, \mathbf{y}, z) \mapsto\left(\mathbf{x}, y_{1}^{n}, \ldots, y_{e}^{n}, y_{1}^{m_{1}} \ldots y_{e}^{m_{e}} z\right)
$$

does not change $Z_{D L}^{\mathcal{W}, \mathbf{y}}\left(h^{t}, \omega, T\right)$. Thus we may suppose that the factor is $\left(z^{n}-\right.$ $\left.\beta t^{\alpha} \mathbf{x}^{\mathbf{n}}\right)^{m}$. Since the computations for the $A$-part depend only on this Newton principal part, it is clear that both terms in the equality differ by an $(\mathbb{L}-1)^{e}$ factor.

Let us consider now the $B$-part. For the sake of simplicity we can suppose that all coordinates of $\mathbf{n}$ are non zero. Following the argument of Step 9, the zeta function corresponding to the $B$-part is the zeta function associated with a power series $\tilde{h}^{t}$ and a form $\tilde{\omega}$ where we must consider arcs based at $\mathbb{A}_{k}^{d} \times \mathbf{G}_{k, m}^{e}$. We can perform a stratification of this space and decompose the result in a sum of zeta functions of families of $\mathcal{W}$-quasi-ordinary power series, where variables corresponding to $J \subset\{1, \ldots, d\}$, pass to $\mathbf{y}$. Since these power series have strictly less depth we can also obtain the $(\mathbb{L}-1)^{e}$ factor.

The Theorems 5.3 and 5.9 are proved.

\section{Consequences of the main theorems}

\subsection{Essential variables.}

Let $h \in k[[\mathbf{x}]][z]$ be a quasi-ordinary power series. For any subset $\emptyset \subsetneq I \subsetneq$ $\{1, \ldots, d\}$, let $k_{I}$ be an algebraic closure of the quotient field of the domain $k\left[\left[x_{i}\right]\right]_{i \in I}$. 
Thus $h_{I} \in k_{I}\left[\left[\widehat{\mathbf{x}}_{I}\right]\right][z]$ is a $k_{I}$-quasi-ordinary power series, where $\widehat{\mathbf{x}}_{I}$ are the variables not in $I$.

Assume $h \in k[[\mathbf{x}]][z]$ is a quasi-ordinary function with $\mathbf{e v}(h)=e<d$. Relabeling the variables we assume that $x_{1}, \ldots, x_{e}$ are the essential variables. Set $I=\{e+$ $1, \ldots, d\}$. Let $\omega=\prod_{l=1}^{d} x_{j}^{\nu_{j}-1} d \mathbf{x} \wedge d z$ be a differential form such that $(h, \omega)$ verifies the support condition 2.3. Whenever the variable $x_{j}$ is not essential then the support condition implies $\nu_{j}=1$. In particular if $\omega_{I}=\prod_{l=1}^{e} x_{j}^{\nu_{j}-1} d \widehat{\mathbf{x}}_{I} \wedge d z$ then the pair $\left(h_{I}, w_{I}\right)$ also verifies the support condition 2.3 over the field $k_{I}$.

Corollary 6.1. In the previous conditions, the following formal identity holds:

$$
Z_{D L}(h, \omega, T)=\mathbb{L}^{-(d+1-e)} Z_{D L}\left(h_{I}, w_{I}, T\right) .
$$

Here formal means that in the RHS, resp. LHS, the varieties are over $k$, resp. $k_{I}$. The proof of the lemma follows essentially from the proof of theorems 5.3 and 5.9 , In fact Newton maps do affect the non-essential variables.

\subsection{Curves case.}

Suppose a $\mathcal{W}$-quasi-ordinary power series $h^{t}$ has $\mathbf{e v}\left(h^{t}\right)=1$. After relabeling the variables $x_{i}$, assume $D_{z}\left(h^{t}\right)=t^{\alpha} x_{1}^{\alpha} \varepsilon(t, \mathbf{x})$, with $\varepsilon(0,0) \neq 0$. We assume the same for the quasi-ordinary power series $h$. Then the compact faces of their Newton polyhedra are contained in 2-dim plane $x_{2}=\ldots=x_{d}=0$. In particular, in the dual space one has the same property. The dual hyperplanes $l_{q, j}^{t}$ are defined by $\alpha_{q, j}+s_{1}^{q, j}\left(b_{1}^{q} v_{1}-n_{1}^{q} v_{d+1}\right)=0$, where $\operatorname{gcd}\left(b_{1}^{q}, n_{1}^{q}\right)=1$. The dual hyperplanes are determined by the linearly independent vectors $\mathbf{w}_{1}^{q}$ and $\mathbf{w}_{l}^{q}=e_{l}$ for $l=2, \ldots, d$. Therefore the sets $G^{q}$ and $G^{q, \alpha_{q, j}}$ have one element. Then we can conclude that

$$
\begin{aligned}
& Z_{D L}^{\mathcal{W}}\left(h^{t}, \omega, T\right)=Z_{A}^{\mathcal{W}}\left(h^{t}, \omega, T\right)+\sum_{q=1}^{r} \sum_{\substack{j=1 \\
s_{1}^{q, j}=1}}^{u(q)} \mathbb{L}^{-\left(\nu_{\mathbf{g}}+A_{\mathbf{g}}\right)} \sum_{w=1}^{w(q, j)} Z_{D L}^{\mathcal{W}}\left(h_{q, j, \mathbf{g}, w}^{t}, \tilde{\omega}_{q, j, \mathbf{g}, w}^{t}, T\right), \\
& Z_{D L}(h, \bar{\omega}, T)=Z_{D L}^{A}(h, \bar{\omega}, T)+\sum_{q=1}^{r} \sum_{\bar{j}=1}^{v(q)} Z_{D L}\left(\bar{h}_{q, \bar{j}}, \omega_{q, \bar{j}}, T\right),
\end{aligned}
$$

where $\nu_{\mathbf{g}}+A_{\mathbf{g}}=\frac{\alpha_{q, j}}{n_{1}^{q}}+\mu_{1}^{\mathbf{g}}\left(p_{1}^{q} \nu_{l}+\bar{b}_{1}^{q}\right)$ and $v(q)=\sum_{j=1}^{u(q)} w(q, j)$.

Corollary 6.2. For a quasi-ordinary power series $h$ with $\mathbf{e v}(h)=1$ and a form $\omega$ such that $(h, \omega)$ verifies condition (2.3) the second line in equations (6.1) can be used as recursive formula to compute $Z_{D L}(h, \bar{\omega}, T)$.

A general formula for the topological zeta function will be given in Theorem 6.3 .

\subsection{The topological zeta function.}

Assume the quasi-ordinary power series $h(\mathbf{x}, z):=\prod_{l=1}^{d} x_{l}^{N_{l}} f(\mathbf{x}, z) u(\mathbf{x}, z), N_{l} \in$ $\mathbb{N}$, is given in a good system of coordinates and that the differential form $\omega=$ $\prod_{j=1}^{d} x_{j}^{\nu_{j}-1} d x_{1} \wedge \ldots \wedge d x_{d} \wedge d z, \nu_{j} \geq 1$, verifies condition (2.3). Assume that $\gamma_{1}, \ldots, \gamma_{r}$ 
(resp. $\left.\tau_{0}, \ldots, \tau_{r}\right)$ are the 1-dimensional (resp. 0-dimensional) compact faces of its Newton polyhedron. Assume for each $q=1, \ldots, r$, on $\gamma_{q}$ there are exactly $v(q)$ distinct roots. Let $\pi_{q, j}$ be the Newton map associated with the Newton component $f_{j}^{q}$ of $f$.

Theorem 6.3. Under the above conditions the following equality holds

$$
\begin{aligned}
Z_{\mathrm{top}, 0}(h, w, s)= & \sum_{l=0}^{r} J_{\tau_{l}}(h, \omega, s)-\sum_{q=1}^{r} v(q) J_{\gamma_{q}}(h, \omega, s)+ \\
& +\sum_{q=1}^{r} \operatorname{mult}\left(\Delta_{\gamma_{q}}\right) \sum_{j=1}^{v(q)} Z_{\mathrm{top}, 0}\left(h \circ \pi_{q, j}, w \circ \pi_{q, j}, s\right) .
\end{aligned}
$$

Moreover if $h$ has non-degenerated principal part then for each $q=1, \ldots, r$ the integer number $v(q)$ is nothing but $V\left(\gamma_{q}\right)$ and for any $j=1, \ldots, v(q)$, the following identity is verified

$$
Z_{\mathrm{top}, 0}\left(h \circ \pi_{q, j}, w \circ \pi_{q, j}, s\right)=\frac{J_{\gamma_{q}}(h, \omega, s)}{(s+1) \operatorname{mult}\left(\Delta_{\gamma_{q}}\right)} .
$$

Proof. The same ideas as in the construction of the above section give $Z_{D L}(h, \omega, T)$ as

$$
Z_{D L}(h, \omega, T)=Z_{D L}^{A}(h, \omega, T)+\sum_{q=1}^{r} \sum_{j=1}^{v(q)} \sum_{\mathbf{g} \in G^{q}} Z^{\gamma_{q}, \mathbf{g}, j}(T),
$$

see equation (5.13). The contribution of the A-part was given in equation (4.16).

Contribution of the $B$-part. Fix a 1-dim face $\gamma_{q}$, a root $\beta_{j}^{q}$ of $\gamma_{q}$ and $\mathbf{g}$ in $G^{q}$.

(1) If $\mathbf{g}=\mathbf{g}^{1}$ then $Z^{\gamma_{q}, \mathbf{g}^{1}, j}(T)=Z_{D L}(\tilde{h}, \tilde{\omega}, T)$, where $\tilde{h}=h \circ \pi_{q, j}$ and $\tilde{\omega}=\omega \circ \pi_{q, j}$, see equation (5.14) in Step 8

(2) If $\mathbf{g} \neq \mathbf{g}^{1}$ then $Z^{\gamma_{q}, \mathbf{g}, j}(T)=Z_{1}^{\gamma_{q}, \mathbf{g}, j}(T)+\sum_{\emptyset \varsubsetneqq J \subset\{1, \ldots, d\}} Z_{J}^{\gamma_{q}, \mathbf{g}, j}(T)$, see Step 9 ,

Recall that the following properties are also verified.

(1) $\chi_{\text {top }}\left(Z_{1}^{\gamma_{q}, \mathbf{g}, j}\left(\mathbb{L}^{-s}\right)\right)=Z_{\text {top }, 0}\left(h \circ \pi_{q, j}, \omega \circ \pi_{q, j}, s\right)$, see equation (5.16).

(2) $\chi_{\text {top }}\left(Z_{J}^{\gamma_{q}, \mathrm{~g}, j}\left(\mathbb{L}^{-s}\right)\right)=0$, see equation (5.17).

Hence the contribution to $Z_{\text {top }, 0}(h, w, s)$ of the $B$-part is

$$
\sum_{q=1}^{r} \sum_{j=1}^{v(q)} \sum_{\mathbf{g} \in G^{q}} Z_{\mathrm{top}, 0}\left(h \circ \pi_{q, j}, \omega \circ \pi_{q, j}, s\right) .
$$

Finally the cardinality of $G^{q}$ is $\operatorname{mult}\left(\Delta_{\gamma_{q}}\right)$.

The second part of the theorem follows from the results of section $\S 3$, in particular from Lemma 3.5 . 
Algorithm. Our method gives an effective algorithm to compute $Z_{\text {top }, 0}(h, w, s)$. After a Newton map $\pi_{q, j}, h \circ \pi_{q, j}\left(\mathbf{y}, z_{1}\right)=y_{1}^{N_{1}} \cdots y_{d}^{N_{d}} \tilde{f}\left(\mathbf{y}, z_{1}\right) \tilde{u}\left(\mathbf{y}, z_{1}\right)$ where $\tilde{f} \in$ $k[[\mathbf{y}]]\left[z_{1}\right]$ is a $z_{1}$-quasi-ordinary power series. Hence $\tilde{f}(\mathbf{0})=0$ and $N_{l}=\inf _{\mathbf{x} \in \Gamma(h)}\left\{\mathbf{w}_{l} \cdot \mathbf{x}\right\}$ for any $l=1, \ldots, d$. Apply again Theorem 6.3 until depth 0 . This algorithm can be implemented in Maple based on a Maple program made by K. Hoornaert and D. Loots for the non-degenerated case, 21.

\subsection{A special candidate pole.}

In this paragraph we will show that some candidate poles disappear when we add their local contributions. For a candidate pole $s_{0}=(N, \nu)$ coming from a compact 1-dimensional face $\gamma$, its local contribution is defined as the sum of the $A$-part of the local Denef-Loeser motivic zeta function which corresponds to $\gamma$ and its two vertices plus the sum of the contributions of the $B$-part. The latter is nothing but the contribution of the highest vertex in the new Newton polyhedron after all Newton maps of Newton components associated with $\gamma$ and at all possible new quasi-ordinary power series.

Consider the following formal power series

$$
\begin{aligned}
h^{t}(\mathbf{x}, z) & :=x_{1}^{N_{1}} \ldots x_{d-1}^{N_{d-1}} z^{n-n_{1} m}\left(z^{n_{1}}-\beta t^{\alpha} x_{1}^{b_{1}} \ldots x_{d-1}^{b_{d-1}} x_{d}\right)^{m}+\ldots, \\
h(\mathbf{x}, z) & :=x_{1}^{N_{1}} \ldots x_{d-1}^{N_{d-1}} z^{n-n_{1} m}\left(z^{n_{1}}-\beta x_{1}^{b_{1}} \ldots x_{d-1}^{b_{d-1}} x_{d}\right)^{m}+\ldots,
\end{aligned}
$$

where the other terms appears behind their Newton polyhedra and $\alpha \in \mathbb{P}, \beta \in \mathbf{G}_{m, k}$. Assume $h^{t} \in k[[t]][[\mathbf{x}]][z]$ is $\mathcal{W}$-quasi-ordinary and $h \in k[[\mathbf{x}]][z]$ is a quasi-ordinary power series.

Consider the differential form $\omega:=x_{1}^{\nu_{1}-1} \ldots x_{d-1}^{\nu_{d-1}-1} d x_{1} \wedge \cdots \wedge d x_{d} \wedge d z$, in such a way $\left(h^{t}, \omega\right)$ and $(h, \omega)$ verify the support condition 2.3 . In other words the pair $\left(N_{d}, b_{d}\right)=(0,1)$. In this section the local contribution of the candidate pole $s_{0}:=$ $1-\mathbb{L}^{-\left(n_{1}+1\right)} T^{n}$ which corresponds with the $d$-th coordinate of the first characteristic exponent of $h^{t}$ and $h$ is computed. Under the above conditions we have the following result.

Proposition 6.4. If there is no pair $\left(N_{i}, b_{i}\right)$ equals to $(0,1)$ other than $\left(N_{d}, b_{d}\right)$ then $s_{0}=1-\mathbb{L}^{-\left(n_{1}+1\right)} T^{n}$ does not appear in the denominator of the local contribution of $s_{0}$ to $Z_{D L}^{\mathcal{W}}\left(h^{t}, \omega, T\right)$ and $Z_{D L}(h, \omega, T)$.

Proof. The compact faces of $\Gamma(h)$ and $\Gamma^{t}\left(h^{t}\right)$ are the same and consist of a face $\gamma$ with vertices $\tau_{0}:=\left(N_{1}+m b_{1}, \ldots, N_{d-1}+m b_{d-1}, m, n-n_{1} m\right)$ and $\tau_{1}:=\left(N_{1}, \ldots, N_{d-1}, 0, n\right)$.

Let $\eta:=b_{1} v_{1}+\ldots b_{d-1} v_{d-1}+v_{d}-n_{1} v_{d+1}$. In the dual space, the dual decomposition induced by $\Gamma(h)$ has only three convex rational cones $\Delta_{\tau_{1}}=\{\eta>0\}, \Delta_{\tau_{0}}=\{\eta<0\}$ and $\Delta_{\gamma}=\{\eta=0\}$. In the same way, the decomposition induced by $\Gamma^{t}(h)$ is given by the convex rational polyhedron $\Delta_{\tau_{1}}^{t}=\{\eta+\alpha>0\}$, and the cones $\Delta_{\tau_{0}}^{t}=\{\eta+\alpha<0\}$ and $\Delta_{\gamma}^{t}=\{\eta+\alpha=0\}$. Since there is only one exponent $\alpha$ of $t$, convex polyhedra delimited by parallel hyperplanes do not exist. 
To compute the contributions of the candidate pole $s_{0}:=1-\mathbb{L}^{-\left(n_{1}+1\right)} T^{n}$ we multiply $Z_{D L}^{\mathcal{W}}\left(h^{t}, \omega, T\right)$ and $Z_{D L}(h, \omega, T)$ by $s_{0}$ and simplify the result under the condition $\mathbb{L}^{-\left(n_{1}+1\right)} T^{n}=1$.

The contribution coming from the Newton polyhedra of $h$ and $h^{t}$ written in terms of generating functions are:

$$
\begin{gathered}
Z_{D L}^{\Delta_{\tau_{0}}^{t}, \mathcal{W}}(T)=T^{m \alpha}(\mathbb{L}-1)^{d+1} \mathbb{L}^{-(d+1)} \Phi_{\Delta_{\tau_{0}}^{t}}(\mathbf{y}), \\
Z_{D L}^{\Delta_{\tau_{1}}^{t}, \mathcal{W}}(T)=(\mathbb{L}-1)^{d+1} \mathbb{L}^{-(d+1)} \Phi_{\Delta_{\tau_{0}}^{t}}(\mathbf{a}), \\
Z_{D L}^{\Delta_{\gamma}^{t}, \mathcal{W}}(T)=\mathbb{L}^{-(d+1)}\left((\mathbb{L}-1)^{d+1}-(\mathbb{L}-1)^{d}\right) \Phi_{\Delta_{\gamma}^{t}}(\mathbf{a}), \\
Z_{D L}^{\Delta_{\tau_{0}}}(T)=(\mathbb{L}-1)^{d+1} \mathbb{L}^{-(d+1)} \Phi_{\Delta_{\tau_{0}}}(\mathbf{y}), \\
Z_{D L}^{\Delta_{\tau_{1}}}(T)=(\mathbb{L}-1)^{d+1} \mathbb{L}^{-(d+1)} \Phi_{\Delta_{\tau_{0}}}(\mathbf{a}), \\
Z_{D L}^{\Delta_{\gamma}}(T)=\mathbb{L}^{-(d+1)}\left((\mathbb{L}-1)^{d+1}-(\mathbb{L}-1)^{d}\right) \Phi_{\Delta_{\gamma}}(\mathbf{a}),
\end{gathered}
$$

where $\mathbf{a}:=\left(a_{1}, \ldots, a_{d-1}, a_{d}, a_{d+1}\right)=\left(\mathbb{L}^{-\nu_{1}} T^{N_{1}}, \ldots, \mathbb{L}^{-\nu_{d-1}} T^{N_{d-1}}, \mathbb{L}^{-1}, \mathbb{L}^{-1} T^{n}\right)$ and $\mathbf{y}:=\left(y_{1}, \ldots, y_{d}, y_{d+1}\right)=\left(\mathbb{L}^{-\nu_{1}} T^{N_{1}+m b_{1}}, \ldots, \mathbb{L}^{-\nu_{d-1}} T^{N_{d-1}+m b_{d-1}}, \mathbb{L}^{-1} T^{m}, \mathbb{L}^{-1} T^{n-n_{1} m}\right)$.

If $\Delta_{1}$ denotes the positive cone $\mathbb{P}^{d+1}$ then the following identities among indicator functions hold: $\left[\Delta_{\tau_{1}}^{t}\right]=\left[\Delta_{1}\right]-\left[\Delta_{\tau_{0}}^{t}\right]-\left[\Delta_{\gamma}^{t}\right]$ and $\left[\Delta_{\tau_{1}}\right]=\left[\Delta_{1}\right]-\left[\Delta_{\tau_{0}}\right]-\left[\Delta_{\gamma}\right]$. Summing and looking only at the terms where $1-\mathbb{L}^{-\left(n_{1}+1\right)} T^{n}$ appears, the contribution of $s_{0}$ is given by

where

$$
\begin{aligned}
& \mathbb{L}^{-(d+1)}(\mathbb{L}-1)^{d+1}\left(-A^{t}(\mathbf{a})+T^{m \alpha} A^{t}(\mathbf{y})-\frac{\mathbb{L}^{-1} \Phi_{\Delta_{\gamma}^{t}}(\mathbf{a})}{\left(1-\mathbb{L}^{-1}\right)}\right), \text { for } h^{t} \text { and } \\
& \mathbb{L}^{-(d+1)}(\mathbb{L}-1)^{d+1}\left(-A(\mathbf{a})+A(\mathbf{y})-\frac{\mathbb{L}^{-1} \Phi_{\Delta_{\gamma}}(\mathbf{a})}{\left(1-\mathbb{L}^{-1}\right)}\right), \text { for } h,
\end{aligned}
$$

$$
A^{t}(\mathbf{x}):=\frac{\sum_{\boldsymbol{\beta} \in G_{0}^{t}} \mathbf{x}^{\boldsymbol{\beta}}}{\left(1-x_{d+1}\right) \prod_{l=1}^{d}\left(1-\mathbf{x}^{\mathbf{w}_{l}}\right)}, \quad A(\mathbf{x}):=\frac{\sum_{\boldsymbol{\beta} \in G_{0}} \mathbf{x}^{\boldsymbol{\beta}}}{\left(1-x_{d+1}\right) \prod_{l=1}^{d}\left(1-\mathbf{x}^{\mathbf{w}_{l}}\right)},
$$

and $G_{0}^{t}$ (resp. $\left.G_{0}\right)$ is the fundamental set of $\Delta_{\tau_{0}}^{t}\left(\right.$ resp. $\left.\Delta_{\tau_{0}}\right)$.

We will compute the terms

$$
A_{1}^{t}:=\left(1-\mathbb{L}^{-\left(n_{1}+1\right)} T^{n}\right)\left(-A^{t}(\mathbf{a})+T^{m \alpha} A^{t}(\mathbf{y})-\frac{\mathbb{L}^{-1} \Phi_{\Delta_{\gamma}^{t}}(\mathbf{a})}{\left(1-\mathbb{L}^{-1}\right)}\right)
$$

and $A_{1}:=\left(1-\mathbb{L}^{-\left(n_{1}+1\right)} T^{n}\right)\left(-A(\mathbf{a})+A(\mathbf{y})-\frac{\mathbb{L}^{-1} \Phi_{\Delta_{\gamma}}(\mathbf{a})}{\left(1-\mathbb{L}^{-1}\right)}\right)$ under the given condition $\mathbb{L}^{-\left(n_{1}+1\right)} T^{n}=1$.

Consider the vertex $\tau_{1}$. The simplification $\mathbb{L}^{-\left(n_{1}+1\right)} T^{n}=1$ gives $a_{d}^{n_{1}} a_{d+1}=1$. Define $w_{j}:=a_{j}^{p_{j}} a_{d+1}^{\bar{b}_{j}}, 1 \leq j \leq d-1$, and $c_{i}:=\operatorname{gcd}\left(n_{1}, b_{i}\right)$ and $p_{i}:=\frac{n_{1}}{c_{i}}, \bar{b}_{i}:=\frac{b_{i}}{c_{i}}$, $i=1, \ldots, d-1$. Thus $w_{j}=\mathbb{L}^{-\left(\nu_{j} p_{j}+\bar{b}_{j}\right)} T^{p_{j} N_{j}+n \bar{b}_{j}}=\mathbb{L}^{\left(b_{j}-\nu_{j}\right) p_{j}} T^{p_{j} N_{j}}$, and $w_{j}=u_{j}^{p_{j}}$ with $u_{j}:=\mathbb{L}^{b_{j}-\nu_{j}} T^{N_{j}}$. 
The elements of $\Delta_{\tau_{0}}$ are written as

$$
\sum_{j=1}^{d-1} \mu_{j}\left(p_{j} e_{j}+\bar{b}_{j} e_{d+1}\right)+\mu_{d}\left(n_{1} e_{d}+e_{d+1}\right)+\mu_{d+1} e_{d+1} \in \mathbb{P}^{d+1}, \mu_{i} \in \mathbb{Q}_{>0} .
$$

The elements of $\Delta_{\tau_{0}}^{t}$ can be written as

$$
\sum_{j=1}^{d-1} \mu_{j}\left(p_{j} e_{j}+\bar{b}_{j} e_{d+1}\right)+\mu_{d}\left(n_{1} e_{d}+e_{d+1}\right)+\mu_{d+1} e_{d+1} \in \mathbb{P}^{d+1}, \mu_{i} \in \mathbb{Q}_{>0}, \mu_{d+1}>\frac{\alpha}{n_{1}} \text {. }
$$

To parametrize the sets $G_{0}^{t}$ and $G_{0}$ we may replace $\mu_{d}$ by $\mu_{d}+s, s \in \mathbb{Z}$, because of the substitution $a_{d}^{-n_{1}}=a_{d+1}$. Thus the elements in $G_{0}^{t}$ (and in $G_{0}$ when $\alpha=0$ ) are obtained with

$$
\left(\mu_{1}, \ldots, \mu_{d-1}, \mu_{d}, \mu_{d+1}\right)=\left(\frac{i_{1}}{p_{1}}, \ldots, \frac{i_{d-1}}{p_{d-1}},-\frac{\alpha+k+\sum_{j=1}^{d-1} i_{j} b_{j}}{n_{1}}, \frac{\alpha+k}{n_{1}}\right),
$$

$i_{j}=1, \ldots, p_{j}, j=1, \ldots, d-1$, and $k=1, \ldots, n_{1}$. Therefore $A^{t}(\mathbf{a})(\operatorname{resp} . A(\mathbf{a}))$ multiplied by $\left(1-a_{d}^{n_{1}} a_{d+1}\right)=1-\mathbb{L}^{-\left(n_{1}+1\right)} T^{n}$ is nothing but:

$$
\begin{gathered}
\frac{\sum_{i_{1}=1}^{p_{1}} \cdots \sum_{i_{d-1}=1}^{p_{d-1}} \sum_{k=1}^{n_{1}} a_{d}^{-\left(\alpha+k+\sum_{j=1}^{d-1} i_{j} b_{j}\right)} \prod_{j=1}^{d-1} a_{j}^{i_{j}}}{\left(1-a_{d+1}\right) \prod_{j=1}^{d-1}\left(1-a_{j}^{p_{j}} a_{d+1}^{\bar{b}_{j}}\right)}=\frac{a_{d}^{-\alpha}\left(\sum_{k=1}^{n_{1}} a_{d}^{-k}\right) \prod_{j=1}^{d-1} \sum_{i_{j}=1}^{p_{j}}\left(a_{j} a_{d}^{-b_{j}}\right)^{i_{j}}}{\left(1-a_{d+1}\right) \prod_{j=1}^{d-1}\left(1-a_{j}^{p_{j}} a_{d+1}^{\bar{b}_{j}}\right)}= \\
=-\frac{a_{d}^{-\alpha}\left(1-a_{d}^{-n_{1}}\right) \prod_{j=1}^{d-1}\left(a_{j} a_{d}^{-b_{j}}\left(1-\left(a_{j} a_{d}^{-b_{j}}\right)^{p_{j}}\right)\right)}{\left(1-a_{d}\right)\left(1-a_{d+1}\right) \prod_{j=1}^{d-1}\left(\left(1-a_{j} a_{d}^{-b_{j}}\right)\left(1-a_{j}^{p_{j}} a_{d+1}^{\bar{b}_{j}}\right)\right)} .
\end{gathered}
$$

The first summand $A^{t}(\mathbf{a})\left(1-a_{d}^{n_{1}} a_{d+1}\right)\left(\right.$ resp. $A(\mathbf{a})\left(1-a_{d}^{n_{1}} a_{d+1}\right)$ when $\left.\alpha=0\right)$ is:

$$
-\frac{a_{d}^{-\alpha} \prod_{j=1}^{d-1} u_{j}}{\left(1-a_{d}\right) \prod_{j=1}^{d-1}\left(1-u_{j}\right)} .
$$

Consider the term from $\gamma$. The elements of $\Delta_{\gamma}^{t}$ (resp. $\Delta_{\gamma}$ when $\alpha=0$ ) are

$$
\sum_{j=1}^{d-1} \mu_{j}^{\prime}\left(p_{j} e_{j}+\bar{b}_{j} e_{d+1}\right)+\ldots+\mu_{d}^{\prime}\left(n_{1} e_{d}+e_{d+1}\right)+\frac{\alpha}{n_{1}} e_{d+1} \in \mathbb{P}^{d+1}, \mu_{i}^{\prime} \in \mathbb{Q}_{>0} .
$$


After simplifying, the fundamental set $G_{\gamma}^{t}$ (resp. $\left.G_{\gamma}\right)$ of $\Delta_{\gamma}^{t}\left(\right.$ resp. $\left.\Delta_{\gamma}\right)$ is obtained from

$$
\left(\mu_{1}^{\prime}, \ldots, \mu_{d-1}^{\prime}, \mu_{d}^{\prime}\right)=\left(\frac{i_{1}}{p_{1}}, \ldots, \frac{i_{d-1}}{p_{d-1}},-\frac{\alpha+\sum_{j=1}^{d-1} i_{j} b_{j}}{n_{1}}\right),
$$

$i_{j}=1, \ldots, p_{j}, j=1, \ldots, d-1$.

Since we have to multiply the factor by $\frac{\mathbb{L}^{-1}}{1-\mathbb{L}^{-1}}$ and $\mathbb{L}^{-1}=a_{d}$, then the product $\left(1-a_{d}^{n_{1}} a_{d+1}\right) \frac{\mathbb{L}^{-1} \Phi_{\Delta_{\gamma}^{t}}(\mathbf{a})}{\left(1-\mathbb{L}^{-1}\right)}$ equals

$$
\begin{gathered}
\frac{a_{d} \sum_{i_{1}=1}^{p_{1}} \cdots \sum_{i_{d-1}=1}^{p_{d-1}} a_{d}^{-\left(\alpha+\sum_{j=1}^{d-1} i_{j} b_{j}\right)} \prod_{j=1}^{d-1} a_{j}^{i_{j}}}{\left(1-a_{d}\right) \prod_{j=1}^{d-1}\left(1-a_{j}^{p_{j}} a_{d+1}^{\bar{b}_{j}}\right)}=\frac{a_{d}^{1-\alpha} \prod_{j=1}^{d-1} \sum_{i_{j}=1}^{p_{j}}\left(a_{j} a_{d}^{-b_{j}}\right)^{i_{j}}}{\left(1-a_{d}\right) \prod_{j=1}^{d-1}\left(1-a_{j}^{p_{j}} a_{d+1}^{\bar{b}_{j}}\right)}= \\
=\frac{a_{d}^{1-\alpha} \prod_{j=1}^{d-1}\left(a_{j} a_{d}^{-b_{j}}\left(1-\left(a_{j} a_{d}^{-b_{j}}\right)^{p_{j}}\right)\right)}{\left(1-a_{d}\right) \prod_{j=1}^{d-1}\left(\left(1-a_{j} a_{d}^{-b_{j}}\right)\left(1-a_{j}^{p_{j}} a_{d+1}^{\bar{b}_{j}}\right)\right)}=\frac{a_{d}^{1-\alpha} \prod_{j=1}^{d-1} u_{j}}{\left(1-a_{d}\right) \prod_{j=1}^{d-1}\left(1-u_{j}\right)} .
\end{gathered}
$$

The term $\left(1-a_{d}^{n_{1}} a_{d+1}\right) \frac{\mathbb{L}^{-1} \Phi_{\Delta_{\gamma}}(\mathbf{a})}{\left(1-\mathbb{L}^{-1}\right)}$ is obtained from equation (6.6) doing $\alpha=0$.

For the vertex $\tau_{0}$, after the simplification, $y_{d}^{n_{1}} y_{d+1}=\mathbb{L}^{-\left(n_{1}+1\right)} T^{n}=1, y_{j} y_{d}^{-b_{j}}=u_{j}$ and $y_{j}^{p_{j}} y_{d+1}^{\bar{b}_{1}}=w_{j}$, for $j=1, \ldots, d-1$. The term $A^{t}(\mathbf{y})$ multiplied by $1-\mathbb{L}^{-\left(n_{1}+1\right)} T^{n}=$ $\left(1-y_{d}^{n_{1}} y_{d+1}\right)$ is the same as $(6.3)$ but $a_{i}$ replaced by $y_{i}$. Therefore the term we are interested in is

$$
-\frac{T^{m \alpha} y_{d}^{-\alpha} \prod_{j=1}^{d-1} u_{j}}{\left(1-y_{d}\right) \prod_{j=1}^{d-1}\left(1-u_{j}\right)}=-\frac{a_{d}^{-\alpha} \prod_{j=1}^{d-1} u_{j}}{\left(1-y_{d}\right) \prod_{j=1}^{d-1}\left(1-u_{j}\right)}
$$

because $T^{-1} y_{d}=a_{d}$. Furthermore $A(\mathbf{y})\left(1-\mathbb{L}^{-\left(n_{1}+1\right)} T^{n}\right)$ is obtained from equation (6.7) when $\alpha=0$. Thus

$$
A_{1}^{t}=(\underline{6.7})-(\underline{6.4})-(\underline{6.6})=-\frac{a_{d}^{-\alpha} y_{d} \prod_{j=1}^{d-1} u_{j}}{\left(1-y_{d}\right) \prod_{j=1}^{d-1}\left(1-u_{j}\right)} .
$$


And substituting $\alpha=0$ in the last identity we get $A_{1}$ :

$$
A_{1}=-\frac{y_{d} \prod_{j=1}^{d-1} u_{j}}{\left(1-y_{d}\right) \prod_{j=1}^{d-1}\left(1-u_{j}\right)} .
$$

Finally we compute the contribution of the $B$ part in the arc decomposition for both power series. Let $\pi_{\gamma}$ be the map defined by $x_{l}=\bar{x}_{l}^{p_{l}}$ and $z=\left(z_{1}+\bar{\beta}\right) \prod_{l=1}^{d} \bar{x}_{l}^{\bar{b}_{l}}$ where $\bar{\beta}^{n_{1}}=\beta$. We compute the contribution after the Newton map $\pi_{\gamma}$ associated with the unique Newton component of $h$ and the corresponding $k[t]$-morphisms associated with each $\mathbf{g} \in G_{\gamma}$. For a pair $(h, \omega)$ in equation (5.19) we show that the contribution is

$$
\begin{gathered}
Z_{D L}(\bar{h}, \bar{\omega}, T)+\sum_{\mathbf{g} \in G_{\gamma}, \mathbf{g} \neq \mathbf{g}^{1}} \mathbb{L}^{-\left(\nu_{\mathbf{g}}+A_{\mathbf{g}}\right)} Z_{D L}^{\mathcal{W}}\left(\tilde{h}_{\mathbf{g}}^{t}, \tilde{\omega}_{\mathbf{g}}, T\right)+ \\
+\sum_{\mathbf{g} \in G_{\gamma}, \mathbf{g} \neq \mathbf{g}^{1}} \mathbb{L}^{-\left(\nu_{\mathbf{g}}+A_{\mathbf{g}}\right)} \sum_{\emptyset \subsetneq J \subset\{1, \ldots, d-1\}}(\mathbb{L}-1)^{\# J} Z_{D L}^{\mathcal{W}}\left(\left(\tilde{h}_{\mathbf{g}}^{t}\right)_{(\mathbf{y}, 0)},\left(\tilde{\omega}_{\mathbf{g}}\right)_{(\mathbf{y}, 0)}, T\right),
\end{gathered}
$$

where the pair $(\bar{h}, \bar{\omega})$ is the pull-back by $\pi_{\gamma}$ of $(h, \omega)$, that is

$$
\bar{h}=\bar{x}_{1}^{\tilde{N}_{1}} \ldots \bar{x}_{d}^{\tilde{N}_{d}}\left(z_{1}^{m_{r}}+\ldots\right) u\left(\overline{\mathbf{x}}, z_{1}\right), \quad u(\mathbf{0}, 0) \neq 0
$$

with $\tilde{N}_{l}=p_{l} N_{l}+\bar{b}_{l} n, l=1, \ldots, d$, and $\bar{\omega}=\bar{x}_{1}^{\tilde{\nu}_{1}-1} \ldots \bar{x}_{d}^{\tilde{\nu}_{d}-1} d \overline{\mathbf{x}} \wedge d z_{1}$.

For $\left(\tilde{h}_{\mathbf{g}}^{t}, \tilde{\omega}_{\mathbf{g}}\right)$ the form $\tilde{\omega}_{\mathbf{g}}$ is nothing but $\bar{\omega}$. Fix $\mathbf{g} \in G_{\gamma} \backslash\left\{\mathbf{g}^{1}\right\}$. The map $\pi_{\gamma} \circ \pi_{\mathbf{g}}$ is $x_{l}=t^{\mu_{l}^{\mathbf{g}} p_{l}} \bar{x}_{l}^{p_{l}}$ and $z=t^{\mathrm{c}}\left(z_{1}+\bar{\beta}\right) \prod_{l=1}^{d} \bar{x}_{l}^{\bar{b}_{l}}$. Then $\tilde{h}_{\mathbf{g}}^{t}=h \circ \pi_{\gamma} \circ \pi_{\mathbf{g}}$, that is

$$
\tilde{h}_{\mathbf{g}}^{t}:=t^{n c^{\mathbf{g}}+\sum_{l=1}^{d-1} \mu_{l}^{\mathbf{g}} p_{l} N_{l}} \bar{x}_{1}^{\tilde{N}_{1}} \ldots \bar{x}_{d}^{\tilde{N}_{d}}\left(z_{1}^{m_{r}}+\ldots\right) u\left(\overline{\mathbf{x}}, z_{1}\right), \quad u(\mathbf{0}, 0) \neq 0 .
$$

For $h^{t}$ the maps associated with the corresponding $k[t]$-morphisms associated with each $\mathbf{g} \in G_{\gamma}^{t}$ appear. Looking at equation (5.18) we have $r=1, u(r)=1$ and $s_{1}^{1,1}=1$. It shows that the contribution is

$$
\begin{gathered}
\sum_{\mathbf{g} \in G_{\gamma}^{t}} \mathbb{L}^{-\left(\nu_{\mathbf{g}}+A_{\mathbf{g}}\right)}\left(Z_{D L}^{\mathcal{W}}\left(\tilde{h}_{\gamma, \mathbf{g}}^{t}, \tilde{\omega}_{\gamma, \mathbf{g}}, T\right)+\right. \\
\left.+\sum_{\emptyset \subsetneq J \subset\{1, \ldots, d-1\}}(\mathbb{L}-1)^{\# J} Z_{D L}^{\mathcal{W}}\left(\left(\tilde{h}_{\gamma, \mathbf{g}}^{t}\right)_{(\mathbf{y}, 0)},\left(\tilde{\omega}_{\gamma, \mathbf{g}}\right)_{(\mathbf{y}, 0)}, T\right)\right) .
\end{gathered}
$$

In this case, once $\mathbf{g} \in G_{\gamma}^{t}$ is fixed, the mapping considered $\pi_{\gamma} \circ \pi_{\mathbf{g}}$ is defined by $x_{l}=t^{\mu_{l}^{\mathbf{g}} p_{l}} \bar{x}_{l}^{p_{l}}$ and $z=t^{c^{\mathbf{g}}}\left(z_{1}+\bar{\beta}\right) \prod_{l=1}^{d} \bar{x}_{l}^{\bar{b}_{l}}$. The pull-back $\tilde{h}_{\gamma, \mathbf{g}}^{t}$ is

$$
t^{n c^{\mathbf{g}}+\sum_{l=1}^{d-1} \mu_{l}^{\mathbf{g}} p_{l} N_{l}} \bar{x}_{1}^{\tilde{N}_{1}} \ldots \bar{x}_{d}^{\tilde{N}_{d}}\left(z_{1}^{m_{r}}+\ldots\right) u\left(\overline{\mathbf{x}}, z_{1}\right), \quad u(\mathbf{0}, 0) \neq 0,
$$

and $\tilde{\omega}_{\gamma, \mathbf{g}}=\bar{\omega}$. 
Computations for (6.10) and for all terms but the first one in (6.9) are similar. In both cases, if either $\mathbf{g} \in G_{\gamma}^{t}$ or $\mathbf{g} \in G_{\gamma} \backslash\left\{\mathbf{g}^{1}\right\}$ are fixed, then the local contribution from $Z_{D L}^{\mathcal{W}}\left(\tilde{h}_{\gamma, \mathbf{g}}^{t}, \tilde{\omega}_{\gamma, \mathbf{g}}, T\right)$ or from $Z_{D L}^{\mathcal{W}}\left(\tilde{h}_{\mathbf{g}}^{t}, \tilde{\omega}_{\mathbf{g}}, T\right)$ we are interested in is given by the $z_{1}$-highest vertex $\tilde{\tau}$ which corresponds in both cases to the monomial

$$
t^{\sum_{l=1}^{d-1} \mu_{l}^{\mathbf{g}} p_{l} N_{l}+n c^{\mathbf{g}}} \bar{x}_{1}^{\tilde{N}_{1}} \ldots \bar{x}_{d}^{\tilde{N}_{d}} z_{1}^{m_{r}} .
$$

We show in Step 6 of the proof of theorems [5.3 and 5.9 that the factor coming from $\tilde{\tau}$ is nothing but $T^{\sum_{l=1}^{d-1} \mu_{l}^{\mathbf{g}} p_{l} N_{l}+n c^{\mathbf{g}}} \mathbb{L}^{-(d+1)}(\mathbb{L}-1)^{d+1} \Phi_{\Delta_{\tilde{\tau}}}(\mathbf{z})$, where

$\mathbf{z}:=\left(\mathbb{L}^{-\left(\nu_{1} p_{1}+\bar{b}_{1}\right)} T^{N_{1} p_{1}+n \bar{b}_{1}}, \ldots, \mathbb{L}^{-\left(\nu_{d-1} p_{d-1}+\bar{b}_{d-1}\right)} T^{N_{d-1} p_{d-1}+n \bar{b}_{d-1}}, \mathbb{L}^{-\left(n_{1}+1\right)} T^{n}, \mathbb{L}^{-1} T^{m}\right)$.

Since the corresponding cone $\Delta_{\tilde{\tau}}^{c}$ is the positive cone $\mathbb{P}^{d+1}$ (whose fundamental set $\tilde{G}$ has only one element $\tilde{G}=\{\mathbf{1}\}$ ), its contribution is

$$
T^{\sum_{l=1}^{d-1} \mu_{l}^{\mathbf{g}} p_{l} N_{l}+n c^{\mathbf{g}}} \mathbb{L}^{-(d+1)}(\mathbb{L}-1)^{d+1} S^{1}(\mathbf{z}),
$$

where

$$
S^{1}(\mathbf{x}):=\frac{\mathbf{x}^{\mathbf{1}}}{\prod_{j=1}^{d+1}\left(1-x_{j}\right)}=\frac{x_{1} x_{2} \ldots x_{d+1}}{\prod_{j=1}^{d+1}\left(1-x_{j}\right)}
$$

Take any $\emptyset \subsetneq J \subsetneq\{1, \ldots, d-1\}$. The number $e$ of essential variables of $\left(\tilde{h}_{\gamma, \mathbf{g}}^{t}\right)_{(\mathbf{y}, 0)}$ and of $\left(\tilde{h}_{\mathbf{g}}^{t}\right)_{(\mathbf{y}, 0)}$ is $e=d+1-\# J$. By Corollary 6.1, $Z_{D L}^{\mathcal{W}}\left(\left(\tilde{h}_{\gamma, \mathbf{g}}^{t}\right)_{(\mathbf{y}, 0)},\left(\tilde{\omega}_{\gamma, \mathbf{g}}\right)_{(\mathbf{y}, 0)}, T\right)$ is $\mathbb{L}^{-\# J}(\mathbb{L}-1)^{e} \mathbb{L}^{-e} T^{\sum_{l=1}^{d-1} \mu_{l}^{\mathbf{g}} p_{l} N_{l}+n c^{\mathbf{g}}} S_{J}^{1}(\mathbf{z})$ where $S_{J}^{1}(\mathbf{x}):=\frac{1}{\left(1-x_{d+1}\right)} \prod_{j \notin J} \frac{x_{j}}{\left(1-x_{j}\right)}$. And the same identity is true for the zeta function $Z_{D L}^{\mathcal{W}}\left(\left(\tilde{h}_{\mathbf{g}}^{t}\right)_{(\mathbf{y}, 0)},\left(\tilde{\omega}_{\mathbf{g}}\right)_{(\mathbf{y}, 0)}, T\right)$

Thus we get all contributions we are interested in for (6.10) adding all previous results:

$$
\mathbb{L}^{-(d+1)}(\mathbb{L}-1)^{d+1} \sum_{\mathbf{g} \in G_{\gamma}^{t}} \mathbb{L}^{-\left(\nu_{\mathbf{g}}+A_{\mathbf{g}}\right)} T^{\sum_{l=1}^{d-1} \mu_{l}^{\mathbf{g}} p_{l} N_{l}+n c^{\mathbf{g}}}\left(S^{1}(\mathbf{z})+\sum_{\emptyset \subsetneq J \subset\{1, \ldots, d-1\}} S_{J}^{1}(\mathbf{z})\right) .
$$

It is proved by induction the formula $S^{1}(\mathbf{x})+\sum_{\emptyset \subsetneq J \subset\{1, \ldots, d-1\}} S_{J}^{1}(\mathbf{x})=S(\mathbf{x})$ where $S(\mathbf{x}):=\frac{x_{d} x_{d+1}}{\prod_{l=1}^{d+1}\left(1-x_{l}\right)}$. Thus we must simplify the contribution

$$
B_{1}^{t}:=\left(1-\mathbb{L}^{-\left(n_{1}+1\right)} T^{n}\right)\left(\sum_{\mathbf{g} \in G_{\gamma}^{t}} \mathbb{L}^{-\left(\nu_{\mathbf{g}}+A_{\mathbf{g}}\right)} T^{\sum_{l=1}^{d-1} \mu_{l}^{\mathbf{g}} p_{l} N_{l}+n c^{\mathbf{g}}} S(\mathbf{z})\right)
$$

under our hypothesis $\mathbb{L}^{-\left(n_{1}+1\right)} T^{n}=1$.

In the other case, equation (6.9), the contribution of $Z_{D L}(\bar{h}, \bar{\omega}, T)$ comes from the $z_{1}$-highest vertex $\tilde{\tau}$ which corresponds to the monomial $\bar{x}_{1}^{\tilde{N}_{1}} \ldots \bar{x}_{d}^{\tilde{N}_{d}} z_{1}^{m_{r}}$. Thus it comes from $\mathbb{L}^{-(d+1)}(\mathbb{L}-1)^{d+1} \Phi_{\Delta_{\tilde{\tau}}}(\mathbf{z})$, where $(\mathbf{z})$ was defined before. In fact since the corresponding cone $\Delta_{\tilde{\tau}}^{c}$ is the positive cone $\mathbb{P}^{d+1}$ then the contribution is $\mathbb{L}^{-(d+1)}\left(\mathbb{L}_{-}\right.$ 
$1)^{d+1} S^{1}(\mathbf{z})$ where $S^{1}(\mathbf{x})$ (cf. equation (6.11)). Thus all contributions after the Newton maps of (6.9) are

$$
\mathbb{L}^{-(d+1)}(\mathbb{L}-1)^{d+1}\left(S^{1}(\mathbf{z})+\sum_{\mathbf{g} \in G_{\gamma}, \mathbf{g} \neq \mathbf{g}^{1}} \mathbb{L}^{-\left(\nu_{\mathbf{g}}+A_{\mathbf{g}}\right)} T^{\sum_{l=1}^{d-1} \mu_{l}^{\mathbf{g}} p_{l} N_{l}+n c^{\mathbf{g}}} S(\mathbf{z})\right) .
$$

We should simplify under our hypothesis $\mathbb{L}^{-\left(n_{1}+1\right)} T^{n}=1$ the formula

$$
B_{1}:=\left(1-\mathbb{L}^{-\left(n_{1}+1\right)} T^{n}\right)\left(S^{1}(\mathbf{z})+\sum_{\mathbf{g} \in G_{\gamma}, \mathbf{g} \neq \mathbf{g}^{1}} \mathbb{L}^{-\left(\nu_{\mathbf{g}}+A_{\mathbf{g}}\right)} T^{\sum_{l=1}^{d-1} \mu_{l}^{\mathbf{g}} p_{l} N_{l}+n c^{\mathbf{g}}} S(\mathbf{z})\right) .
$$

Recall that the $(d+1)$-tuple $\mathbf{z}$ is nothing but $\left(w_{1}, \ldots, w_{d-1}, 1, y_{d}\right)$.

Let us study the case $B_{1}^{t}$. For every $\mathbf{g}$ parametrized by $\left(i_{1}, \ldots, i_{d-1}\right)$ from equation (6.5) we have the following identity, which is only valid after the simplification we are doing:

$$
\nu_{\mathbf{g}}+A_{\mathbf{g}}=\frac{\alpha}{n_{1}}+\sum_{j=1}^{d-1} \frac{i_{j}}{p_{j}}\left(p_{j} \nu_{j}+\bar{b}_{j}\right)-\frac{\alpha+\sum_{j=1}^{d-1} i_{j} b_{j}}{n_{1}}\left(n_{1}+1\right) .
$$

It is turn out $\nu_{\mathbf{g}}+A_{\mathbf{g}}=-\alpha-\sum_{j=1}^{d-1} i_{j}\left(b_{j}-\nu_{j}\right)$. Since $c^{\mathbf{g}}=\frac{\alpha}{n_{1}}+\sum_{l=1}^{d} \mu_{l} \bar{b}_{l}$ then, under our simplification, $c^{\mathbf{g}}=0$ and the exponent of $T$ is nothing but $\sum_{j=1}^{d-1} i_{j} N_{j}$.

Therefore $B_{1}^{t}$ equals:

$$
\frac{\mathbb{L}^{\alpha} z_{d+1} \prod_{j=1}^{d-1} \sum_{k=1}^{p_{j}}\left(\mathbb{L}^{\left(b_{j}-\nu_{j}\right)} T^{N_{j}}\right)^{k}}{\left(1-z_{d+1}\right) \prod_{j=1}^{d-1}\left(1-z_{j}\right)}=\frac{\mathbb{L}^{\alpha} z_{d+1} \prod_{j=1}^{d-1}\left(u_{j}\left(1-u_{j}^{p_{j}}\right)\right)}{\left(1-z_{d+1}\right) \prod_{j=1}^{d-1}\left(\left(1-z_{j}\right)\left(1-u_{j}\right)\right)} .
$$

Written as before we have

$$
B_{1}^{t}=\frac{a_{d}^{-\alpha} y_{d} \prod_{j=1}^{d-1} u_{j}}{\left(1-y_{d}\right) \prod_{j=1}^{d-1}\left(1-u_{j}\right)} .
$$

Thus

$$
A_{1}^{t}+B_{1}^{t}=(6.7)-(6.4)-(6.6)+(6.12)=0 .
$$

Let us study the case $B_{1}$. For every $\mathbf{g} \neq \mathbf{g}^{1}$ parametrized by $\left(i_{1}, \ldots, i_{d-1}\right)$ from equation (6.5) but now $i_{j} \in\left\{1, \ldots, p_{j}-1\right\}$, we have $\nu_{\mathbf{g}}+A_{\mathrm{g}}=-\sum_{j=1}^{d-1} i_{j}\left(b_{j}-\nu_{j}\right)$. In the same way the exponent $T$ is nothing but $\sum_{j=1}^{d-1} i_{j} N_{j}$. 
The first term of $B_{1}$ is

$$
\left(1-z_{d}\right) S^{1}(\mathbf{z})=\frac{y_{d} \prod_{j=1}^{d-1} w_{j}}{\left(1-y_{d}\right) \prod_{j=1}^{d-1}\left(1-w_{j}\right)}=\frac{y_{d} \prod_{j=1}^{d-1} u_{j}^{p_{j}}}{\left(1-y_{d}\right) \prod_{j=1}^{d-1}\left(1-u_{j}^{p_{j}}\right)} .
$$

The other terms comes from

$$
\frac{z_{d+1}}{\left(1-z_{d+1}\right) \prod_{j=1}^{d-1}\left(1-z_{j}\right)} \prod_{j=1}^{d-1} \sum_{k=1}^{p_{j}-1}\left(\mathbb{L}^{\left(b_{j}-\nu_{j}\right)} T^{N_{j}}\right)^{k}
$$

This is nothing but

$$
\frac{y_{d}}{\left(1-y_{d}\right) \prod_{j=1}^{d-1}\left(1-u_{j}^{p_{j}}\right)} \prod_{j=1}^{d-1} \sum_{k=1}^{p_{j}-1}\left(u_{j}\right)^{k}=\frac{y_{d}}{\left(1-y_{d}\right) \prod_{j=1}^{d-1}\left(1-u_{j}^{p_{j}}\right)^{d-1}} u_{j=1}\left(\frac{1-u_{j}^{p_{j}}}{1-u}-u_{j}^{p_{j}-1}\right) .
$$

Thus $A_{1}+B_{1}=(6.8)+(6.13)+(6.14)=0$.

Let $h \in k[[\mathbf{x}]][z]$ be a quasi-ordinary power series in good coordinates with $h(\mathbf{x}, z)=x_{1}^{N_{1}} x_{2}^{N_{2}} \ldots x_{d}^{N_{d}} g(\mathbf{x}, z)$, where no $x_{i}$ divides $g(\mathbf{x}, z)$ and $N_{l} \geq 0$ for any $l=1, \ldots, d$. Write $h(\mathbf{x}, z)=x_{1}^{N_{1}} x_{2}^{N_{2}} \ldots x_{d}^{N_{d}} f(\mathbf{x}, z) u(\mathbf{x}, z)$, where $f(\mathbf{x}, z)$ is a quasiordinary $z$-polynomial of degree $n$ in $k[[\mathbf{x}]][z]$. Let $\omega=\prod_{j=1}^{d} x_{j}^{\nu_{j}-1} d x_{1} \wedge \ldots \wedge d x_{d} \wedge$ $d z, \nu_{j} \geq 1$ define a form such that $(h, \omega)$ verifies the support condition (2.3). The set $C P(h, \omega)$ of candidate poles was defined in (4.25). Proposition 6.4 will allow to consider a smaller set of candidate poles than the set $C P(h, \omega)$.

Definition 6.5. A compact 1-dimensional face $\gamma_{q}$ in $N D(h)$ will be called special in the $i$-th coordinate if the pair $\left(h_{\gamma_{q}}, \omega\right)$ verifies the conditions of Proposition 6.4 in the $i$-th coordinate (instead of $d$-th coordinate). The face $\gamma_{q}$ will be called special if it is special in one of the coordinates. If $\gamma_{q}$ is special in the $i$-th and $j$-th, $(i \neq j)$, coordinates then the corresponding candidate poles coincide. Define

$$
C P(h, \omega)_{q}:=\left\{\left(\frac{M_{l}^{q}}{c_{l}^{q}}, \nu_{l} p_{l}^{q}+\bar{b}_{l}^{q}\right)\right\}_{l=1, \ldots, \hat{i}, \ldots, d} \text { if } \gamma_{q} \text { is special, }
$$

where we assume that $\gamma_{q}$ is special in the $i$-th coordinate and $\widehat{i}$ means that we omit $i$. In particular, if $\gamma_{q}$ is special in more than one variable then the corresponding pair $(N, \nu)$ appears in $C P(h, \omega)_{q}$. Otherwise, define

$$
C P(h, \omega)_{q}:=\left\{\left(\frac{M_{l}^{q}}{c_{l}^{q}}, \nu_{l} p_{l}^{q}+\bar{b}_{l}^{q}\right)\right\}_{l=1}^{d} \text { if } \gamma_{q} \text { is not special. }
$$


Let us define by induction the sets

$$
\widetilde{C P}(h, \omega):=\bigcup_{q=1}^{r} C P(h, \omega)_{q} \cup \bigcup \widetilde{C P}\left(\bar{h}_{q, j}, \bar{\omega}_{q, j}\right)
$$

where the union is over all pull-back $\left(\bar{h}_{q, j}, \bar{\omega}_{q, j}\right)$ under the Newton maps associated with Newton components $f_{j}^{q}$ of $f$. The set of strong candidate poles of a pair $(h, \omega)$ is defined by

$$
\operatorname{SCP}(h, \omega):=\left\{\left(N_{i}, \nu_{i}\right)\right\}_{i=1}^{d} \cup \widetilde{C P}(h, \omega) .
$$

After Proposition 6.4 and Theorem 6.3 the following result is clear.

Proposition 6.6. If $N s+\nu$ is a pole of $Z_{\text {top }, 0}(h, \omega, s)$ then $(N, \nu) \in S C P(h, \omega)$.

In fact Proposition 6.4 and equations (5.18) and (5.19) show that the same result is true for the local Denef-Loeser motivic zeta function.

\section{Proposition 6.7.}

$$
Z_{D L}(h, \omega, T) \in \mathbb{Z}\left[\mathbb{L}, \mathbb{L}^{-1},\left(1-\mathbb{L}^{-\nu} T^{N}\right)^{-1}\right][T]_{(N, \nu) \in S C P(h, \omega)} .
$$

Remark 6.8. Let $h=\prod_{l=1} x_{l}^{N_{l}} f(\mathbf{x}, z) u(\mathbf{x}, z)$, with $u(\mathbf{0}, 0) \neq 0$. Take any compact 1-dimensional face $\gamma$ of $\Gamma(h)$. For each $j \in\{1, \ldots, d\}$ such that $\gamma$ is not contained in the $\left(x_{j}, z\right)$-plane, consider the $j$-transversal section $h_{j}^{0}$ with root $\alpha=0$. Let $\tilde{\gamma}$ be the compact face of $\Gamma\left(h_{j}^{0}\right)$ on which $\gamma$ is projected. The face $\tilde{\gamma}$ can be the projection of several distinct compact faces of $\Gamma(h)$. Thus if $\gamma$ is not special then its projection $\tilde{\gamma}$ cannot be special in any coordinate.

Strong candidate poles for ev $(h)=1$. Assume $h=x_{1}^{N_{1}} f\left(x_{1}, z\right) u\left(x_{1}, z\right) \in$ $k\left[\left[x_{1}\right]\right][z]$ is a quasi-ordinary power series in good coordinates with $\mathbf{e v}(h)=1$. Let $\gamma$ be a compact 1-dim face of $\Gamma(h)$. Then $\gamma$ is special if and only if $\gamma$ is the highest (with respect to $z)$ compact face of $\Gamma(h)$ and $h_{\gamma}$ is of type $z^{n-n_{1} m}\left(z^{n_{1}}-\beta x_{1}\right)^{n-n_{1}}, \beta \in \mathbf{G}_{m, k}$. In particular $N_{1}=0$. We observe that $\gamma$ is special if and only we can apply inversion formula to $h$ permuting coordinates. If $\gamma$ is special then $C P(h, \omega)_{\gamma}=\emptyset$, for each pair $(h, \omega)$ verifying the support condition (2.3). After a Newton map associated with a Newton component of $f$, the pull-back of $h$ is of type $y^{a} g\left(y, z_{1}\right)$ with $a>0$. In particular Proposition 6.4 cannot be applied anymore.

If $\mathbf{e v}(h)>1$, more general facts occur. Take for instance any of the following examples: $f(x, y, z)=\left(z^{2}-x^{3}\right)^{2}+x^{11} y$ and $g(x, y, u, z)=\left(\left(z^{2}-x^{3} y\right)^{2}+x^{7} y^{2}\right)\left(\left(z^{1}\right) 2-\right.$ $\left.\left.x^{3} y u\right)^{2}+x^{7} y^{2} u^{3}\right)$. For $f$, after the unique possible Newton map $\pi$, a special face for $f \circ \pi$ appears. In the other case, $\Gamma(g)$ has two compact 1-dimensional faces and both are special, in different coordinates.

Strong candidate poles for $\mathbf{e v}(h)=2$. Let $h \in k\left[\left[x_{1}, x_{2}\right]\right][z]$ be a quasi-ordinary power series in good coordinates with $\mathbf{e v}(h)=2$. Assume that we can decompose $h=x_{1}^{N_{1}} x_{2}^{N_{2}} f\left(x_{1}, x_{2}, z\right) u\left(x_{1}, x_{2}, z\right)$, with $u(\mathbf{0}) \neq 0$ and $f$ is a Weierstrass polynomial of degree $n$. Let $\gamma$ denote the highest (with respect to $z$ ) compact face of $\Gamma(h)$ and let $h_{\gamma}=x_{1}^{N_{1}} x_{2}^{N_{2}} z^{n-n_{1} m} \prod_{j=1}^{u}\left(z^{n_{1}}-\beta_{j} x_{1}^{b_{1}} x_{2}^{b_{2}}\right)^{m_{j}}$ with $\beta_{j} \in \mathbf{G}_{m, k}$ and $m=\sum m_{j}$. Let $\omega=x_{1}^{\nu_{1}-1} x_{2}^{\nu_{2}-1} d x_{1} \wedge d x_{2} \wedge d z$ be a differential form such that $(h, \omega)$ verifies (2.3). 
Lemma 6.9. $\left(n, n_{1}+1\right) \in C P(h, w)_{\gamma}$ if and only if either

(1) there exists $i \in\{1,2\}$ such that $b_{i}=1, N_{i}=0$ (this implies $\nu_{i}=1$ ) and $u>1$, or

(2) $u=1$ and $b_{1}=b_{2}=1, N_{1}=N_{2}=0$ (this implies $\nu_{1}=\nu_{2}=1$ ) and therefore $\gamma$ is special in both coordinates.

Proof. By definitions and Lemma 4.14, $\gamma$ is defined by $n_{1} x_{1}+b_{1} z=n_{1} N_{1}+b_{1} n$ and $n_{1} x_{2}+b_{2} z=n_{2} N_{2}+b_{2} n$. The candidate poles from $\gamma$ are $\left(\frac{n_{1} N_{i}+b_{i} n}{\operatorname{gcd}\left(n_{1}, b_{i}\right)}, \frac{n_{1} \nu_{i}+b_{i}}{\operatorname{gcd}\left(n_{1}, b_{i}\right)}\right)$ with $i=1,2$. In particular $\left(\frac{n_{1} N_{i}+b_{i} n}{\operatorname{gcd}\left(n_{1}, b_{i}\right)}, \frac{n_{1} \nu_{i}+b_{i}}{\operatorname{gcd}\left(n_{1}, b_{i}\right)}\right)=\left(n, n_{1}+1\right)$ if and only if either $b_{i}=0$ and then $\operatorname{gcd}\left(n_{1}, b_{i}\right)=n_{1}$, which implies $\left(N_{i}, \nu_{i}\right)=\left(n, n_{1}+1\right) \in C P(h, w)_{\gamma}$ which is absurd. Or one of the two conditions of the lemma is verified.

In case $(1)$, since $u>1,\left(n, n_{1}+1\right)$ is a strong candidate pole for $\left.h_{j}^{\alpha}, j \neq i\right)$. In case (2) the $i$-transversal section $h_{i}^{\alpha}$ of $h$ has only $\alpha=0$ as a root because $\left(\frac{1}{n}, \frac{1}{n}\right)$ is the smallest characteristic exponent of $f$, it is the z-highest face of $N P(h)$. In particular $h_{i}^{0}$ has at least one Newton component associated with the projection $\tilde{\gamma}$ of $\gamma$ on the plane $x_{i} z$. Thus $\left(n, n_{1}+1\right)$ is a candidate pole for $\left(h_{i}^{0}, \omega_{i}\right)$. In fact, $\tilde{\gamma}$ is the highest face with respect to $z$. Applying the case $\mathbf{e v}(h)=1$ then $\left(n, n_{1}+1\right)$ is not a strong candidate pole for both $h_{i}^{0}$.

Proposition 6.10. Under the above conditions one has

$$
S C P(h, \omega) \subset \bigcup_{i=1}^{2} \bigcup_{m=1}^{v_{i}} S C P\left(h_{i}^{\alpha_{m}}, \omega_{i}\right) \cup\left\{\left(n, n_{1}+1\right)\right\},
$$

where $h_{i}^{\alpha_{m}}$ are the corresponding $i$-transversal section at the root $\alpha_{m}$, cf. equation 4.19) in Proposition 4.35.

Proof. Let us prove by induction on $\operatorname{depth}(h)$, that if we are in good coordinates, with the above definitions of $n, n_{1}$ and $\gamma$ then

$$
S C P(h, \omega) \subset \bigcup_{i=1,2} \bigcup_{m=1}^{v_{i}} S C P\left(h_{i}^{\alpha_{m}}, \omega_{i}\right) \cup\left\{\left(n(h), n_{1}(h)+1\right)\right\} .
$$

We write $\left(n(h), n_{1}(h)+1\right)$ to remark that at each step of the induction they depend on $h$.

If $\operatorname{depth}(h)=0$ then $h=x_{1}^{N_{1}} x_{2}^{N_{2}} z u(\mathbf{x}, z)$, with $u(\mathbf{0}) \neq 0$ The result follows easily. Assume that we have proved the result for $\operatorname{depth}(h)<m$. Let us decompose $h=x_{1}^{N_{1}} x_{2}^{N_{2}} f\left(x_{1}, x_{2}, z\right) u\left(x_{1}, x_{2}, z\right)$, with $u(\mathbf{0}) \neq 0$ and $\operatorname{depth}(h)=m$. Let $(N, \nu) \in$ $S C P(h, \omega) \subset C P(h, \omega)$. If $(N, \nu)=\left(n, n_{1}+1\right)$ we are done. If $(N, \nu)=\left(N_{i}, \nu_{i}\right) \neq$ $(0,1), i=1,2$ then any of the factors $h_{j}^{\alpha}$ of the $j$-transversal section with $i \neq j$ has $x_{i}^{N_{i}}$ as a factor which implies $(N, \nu)=\left(N_{i}, \nu_{i}\right) \in \operatorname{SCP}\left(h_{j}^{\alpha}, \omega_{j}\right)$.

If $(N, \nu) \in \bigcup_{q=1}^{r} C P(h, \omega)_{q}$ where $\Gamma(h)$ has $r$ compact 1-dim faces. Assume $(N, \nu)$ appears in the compact face $\gamma_{q}$, say in the $i$-th coordinate. Take the transversal section $h_{j}^{0}, j \neq i$. Let $\tilde{\gamma}$ be the compact face of $\Gamma\left(h_{j}^{0}\right)$ on which $\gamma_{q}$ is projected. 
If $\gamma_{q}$ is contained in the $\left(x_{i}, z\right)$-plane, then $\tilde{\gamma}$ contains $\gamma_{q}$. If we can apply proposition 6.4 to $h_{j}^{0}$ and $\tilde{\gamma}$ then $\left.\left(h_{j}^{0}\right)\right|_{\tilde{\gamma}}=z^{n-m n_{q}}\left(z^{n_{q}}-\beta x_{i}\right)^{m}, \beta \in \mathbf{G}_{m, k_{j}}$. Then $\gamma_{q}$ must corresponds to the smallest characteristic exponent of $h$. Thus $\gamma_{q}$ is special in this coordinate. This contradicts $(N, \nu) \in S C P(h, \omega)$.

Otherwise, Remark 6.8 contradicts $(N, \nu) \in C P(h, \omega)_{q}$ if $\gamma_{q}$ is not special in the $i$ th coordinate. If $\gamma_{q}$ is special in the $i$-th coordinate but not in the $j$-coordinate then $(N, \nu) \notin C P(h, \omega)_{q}$ which is absurd. Therefore if $\gamma_{q}$ is special in both coordinates then $\gamma_{q}$ is the highest compact face and by Lemma 6.9 $(2),(N, \nu)=\left(n, n_{1}+1\right)$. Finally we apply induction.

To finish the proof of the proposition is enough to remark that the conditions in Lemma 6.9 (2) cannot be obtained after any Newton map. The reason for that is that after any Newton map the pull-back of $h$ has at least a factor of type $y_{i}^{N_{i}}$ with $N_{i}>0$. Therefore the proof is finished.

Strong candidate poles for $\mathbf{e v}(h)=d>2$. Let $h \in k\left[\left[x_{1}, \ldots, x_{d}\right]\right][z]$ be a quasi-ordinary power series in good coordinates with $\mathbf{e v}(h)=d>2$. Assume that $h=\prod_{l=1}^{d} x_{l}^{N_{l}} f(\mathbf{x}, z) u(\mathbf{x}, z)$, with $u(\mathbf{0}) \neq 0$ and $f$ is a Weierstrass polynomial of degree $n$. Let $\omega$ be a differential form such that $(h, \omega)$ verifies the support condition 2.3 .

Proposition 6.11. Under the above conditions one has

$$
S C P(h, \omega) \subset \bigcup_{i=1, \ldots, d, m=1}^{v_{i}} \bigcup_{m} S P\left(h_{i}^{\alpha_{m}}, \omega_{i}\right),
$$

where $h_{i}^{\alpha_{m}}$ are the corresponding $i$-transversal section at the root $\alpha_{m}$, see lemma 4.19.

Proof. The proof is by induction on the $\operatorname{depth}(h)$ and follows the same ideas as proof of proposition 6.10 but in this case we do not need to take care of the special compact 1-dimensional faces.

Complex analytic set up. If $k=\mathbb{C}$ and we work with convergent complex quasiordinary power series $h \in \mathbb{C}\{\mathbf{x}\}[z]$ all results presented in the last three sections are valid too. We leave the details to the reader. The pull-back under the Newton maps of convergent quasi-ordinary power series are again convergent. The transversal sections can be seen now as follows. We write $h(\mathbf{x}, z)=\prod_{l=1}^{d} x_{l}^{N_{l}} f(\mathbf{x}, z) u(\mathbf{x}, z)$, where no $x_{i}$ divides $f(\mathbf{x}, z), u(0,0) \neq 0, N_{l} \geq 0$ for any $l=1, \ldots, d$, and $f$ is a Weierstrass polynomial in $z$. After Lemma 6.1] we may assume that $\mathbf{e v}(h)=d$. We may assume $f$ is convergent in a polydisk $\Delta_{\varepsilon}^{d} \times \Delta_{\delta}^{1}$, where $0 \ll \delta \ll \varepsilon \ll 1$ and if $\mathbf{x}^{0} \in \Delta_{\varepsilon}^{d}$ all roots of $f\left(\mathbf{x}^{0}, z\right)$ lie in $\Delta_{\delta}^{1}$.

Let us consider the germ $(\operatorname{Sing}(V), 0)$ of singular points of $V=h^{-1}(0)$ in a neighborhood of the origin. The condition of the discriminant implies that $(\operatorname{Sing}(V), 0)$ is contained in the intersection of $h^{-1}(0)$ with the hyperplanes $x_{l}=0,1 \leq l \leq d$. Fix $i \in\{1, \ldots, d\}$. Consider the polynomial, over $\mathbb{C}\left\{x_{i}\right\}$, of degree $n$

$$
f\left(\mathbf{0}_{i}\left(x_{i}\right), z\right)=z^{n}+\text { lower degree terms. }
$$


where the $d$-tuple $\mathbf{0}_{i}$ has all coordinates 0 but the $i$-th coordinate which is $x_{i}$. We have two possibilities:

(1) If $f\left(\mathbf{0}_{i}\left(x_{i}\right), z\right)=z^{n}$ then the points $\left(\mathbf{0}_{i}\left(x^{0}\right), 0\right)$ belong to $h^{-1}(0)$, for all $x^{0} \in$ $\Delta_{\varepsilon}^{1}$. The power series $h_{i}^{0, x^{0}}(\mathbf{x}, z):=h\left(\mathbf{x}+\mathbf{0}_{i}\left(x^{0}\right), z\right)$ are quasi-ordinaries. They are the analytic equivalent to the formal $i$-transversal section at the root $\alpha=0$.

(2) Otherwise, i.e. if $f\left(\mathbf{0}_{i}\left(x_{i}\right), z\right) \neq z^{n}$, given $x^{0} \in \Delta_{\varepsilon}^{1}$ there exist $z_{1}^{0}, \ldots, z_{l}^{0} \in \Delta_{\delta}^{1}$, pairwise distinct, and $r_{1}, \ldots, r_{l} \in \mathbb{P}, \sum r_{j}=n$, such that

$$
f\left(\mathbf{0}_{i}\left(x^{0}\right), z\right)=\prod_{j=1}^{l}\left(z-z_{j}^{0}\right)^{r_{j}} .
$$

We can suppose that $\varepsilon$ is small enough in order to have that $r_{1}, \ldots, r_{l}$ independent of $x^{0}$. Again $h_{i}^{\alpha, x^{0}}(\mathbf{x}, z):=h\left(\mathbf{x}+\mathbf{0}_{i}\left(x^{0}\right), z+z_{j}^{0}\right)$ are quasi-ordinary power series. In this case they are the equivalent in the analytic case to the $i$-transversal at some root $\alpha \neq 0$.

In particular we have the analytic analogue of proposition 4.35.

\section{MONODROMY CONJECTURE FOR QUASI-ORDINARY POWER SERIES}

Let $h:\left(\mathbb{C}^{d+1}, 0\right) \rightarrow(\mathbb{C}, 0)$ be a germ of complex analytic function such that $h(0)=$ 0 . Fix $U$ a sufficiently small neighbourhood of 0 where $h$ is defined. Let $F$ be the Milnor fibre of the Milnor fibration at the origin associated with $h$. Let $m_{F}: F \rightarrow F$ be the monodromy transformation. The zeta-function of the monodromy of $h$ is

$$
\zeta(h)(t):=\prod_{q \geq 0} \operatorname{det}\left(I-t m_{F q}\right)^{(-1)^{q}}
$$

where $m_{F q}: H_{q}(F, \mathbb{C}) \rightarrow H_{q}(F, \mathbb{C}), q \geq 0$, are the homological monodromy transformations.

In this section we solved in the quasi-ordinary case the monodromy conjectures stated in the Introduction. We will focus firstly on the the topological monodromy conjecture of [12] and on the motivic monodromy conjecture of [13, section 2.4] as stated in the introduction but for convergent quasi-ordinary series.

The monodromy conjectures deal with eigenvalues of the complex algebraic monodromy at some points of the zero locus of $h$. First we will prove the monodromy conjecture for $\mathbf{e v}(h)=1$. As we mention in the introduction the result is known (several proofs by Loeser, Veys) but we present here an independent proof following our ideas which by the way will be useful for the proof in the general case. In the general case, i.e $\mathbf{e v}(h)>1$, we need to consider the monodromy at some different points of the singular locus of $h$. These points will be at some transversal sections of $h$. Thus we follow by induction on $\mathbf{e v}(h)$.

\subsection{Monodromy conjecture for curves.}

Recall that any germ $h \in \mathbb{C}\{x, z\}$ of curve is quasi-ordinary. We will prove that each Newton process produces eigenvalues of the zeta function of the monodromy 
of the curve defined by $h$. This is true in all cases except for the special candidate pole but the special pole candidate (if it appears) is not a pole for the motivic zeta function (hence for the topological zeta function) because of Proposition 6.4.

Theorem 7.1. Let $h(x, z) \in \mathbb{C}\{x\}[z]$ be a germ of curve and $\omega$ a regular differential form such that $(h, \omega)$ satisfies the support condition (2.3). If $(N, \nu) \in \operatorname{SCP}(h, \omega)$ let $q:=-\frac{\nu}{N}$, then $\exp (2 i \pi q)$ is an eigenvalue of the zeta function of the monodromy at some point of $h^{-1}(0)$.

Proof. The case $\operatorname{depth}(h)=0$ is trivial, since $h=x^{N} z$. In this case $\operatorname{SCP}(h)=$ $\{(N, \nu),(1,1)\}$, where $\nu$ comes from the differential form; at some points of $h^{-1}(0)$, the zeta function of the monodromy is either $1-t^{N}$ or $1-t$ and the result follows.

We prove by induction on the depth $\geq 1$ that if $(N, \nu) \in S C P(h, \omega)$ and $q:=-\frac{\nu}{N}$, then $\exp (2 i \pi q)$ is an eigenvalue of the zeta function of the monodromy. We will not consider the case $q \in \mathbb{Z}$ since in this case it gives always the eigenvalue 1 of the monodromy.

Let $h$ be a curve singularity with depth $m+1$ in good coordinates, $m \geq 0$. Assume $h=x^{N} z^{\varepsilon} g(x, z), \varepsilon=0,1$ and $\omega=x^{\nu-1} d x \wedge d z$, where neither $x$ nor $z$ divide $g$ and $(h, \omega)$ verifies the support condition 2.3. Let $\gamma_{1}, \ldots, \gamma_{r}$ be the compact edges of the Newton diagram of $h$; for each $q=1, \ldots, r$, we have denoted by $v(q)$ the number of non-zero distinct roots of $h_{\gamma_{q}}$. Each one of these roots define a Newton map and let $h_{q, j} j=1, \ldots, v(q)$, be the pull-backs of $h$ by these Newtons maps. Recall that the vertex $\tau_{r}$ of $\gamma_{r}$ is the $z$-highest vertex. We will define also $\alpha, \beta$ such that $\alpha=0$ (resp. $\beta=0$ ) if the slope of $\gamma_{r}$ (resp. the inverse of the slope of $\gamma_{1}$ ) is an integer and $x$ (resp. $z$ ) does not divide $h$. Otherwise we set $\alpha=1$ (resp. $\beta=1$ ). Besides the candidate poles $(N, \nu)$ and $(1,1)$, denote by $\left(N_{q}, \nu_{q}\right), q=1, \ldots, r$, the candidate poles corresponding to the edges. It is clear that with the poles $(N, \nu)$ and $(1,1)$ we can argue as above to conclude. Note that:

- $\operatorname{depth}(h)=1$ if and only if $\operatorname{depth}\left(h_{q, j}\right)=0$; the corresponding monodromy zeta functions for these pull-backs are equal to 1.

- $h$ is in good coordinates if and only if $v(1)+\beta>1$.

- $\left(N_{r}, \nu_{r}\right)$ is a strong candidate pole if and only if $v(r)+\alpha>1$; recall that $\left(N_{q}, \nu_{q}\right), q=1, \ldots, r-1$, are strong candidate poles.

One can prove, see e.g. 5], that at the corresponding points of the strict transform of $h$ after Newton mappings and after the partial resolution induced by the Newton polygon of $h$ the total transforms of $h$ are isomorphic curves. Using A'Campo's formula we deduce that the monodromy zeta function of $h$ at the origin is

$$
\zeta(h)(t)=\frac{\left(1-t^{N_{r}^{\prime}}\right)^{a}}{\left(1-t^{N_{r}}\right)^{\alpha-1}} \frac{\left(1-t^{N_{1}^{\prime \prime}}\right)^{b}}{\left(1-t^{N_{1}}\right)^{\beta-1}} \prod_{q=1}^{r}\left(\left(1-t^{N_{q}}\right)^{-v(q)} \prod_{j=1}^{v(q)} \zeta\left(h_{q, j}\right)(t)\right),
$$

where

- $N_{1}^{\prime}$ divides $N_{1}, N_{r}^{\prime \prime}$ divides $N_{r}$.

- If $x$ (resp. $z$ ) divides $h$ then $a=0$ (resp. $b=0$ ) and the corresponding numerator does not appear, otherwise $a=1$ (resp. $b=1$ ). 
- If $x$ does not divide $h$, it implies $(N, \nu)=(0,1)$, we can express $h_{\gamma_{r}}=z^{u_{r}} \tilde{h}_{r}$ where $\tilde{h}_{r}$ is a product of $m_{r}=\sum_{j=1}^{v(r)} m_{r, j}$ factors of the form $\left(z^{n_{1}^{r}}-v x^{b^{r}}\right)$, counting multiplicities. Recall that in the case of curves we have always $\operatorname{gcd}\left(n_{1}^{r}, b^{r}\right)=1$. Thus

$$
N_{r}=\left(u_{r}+m_{r} n_{1}^{r}\right) b^{r}, \quad \nu_{r}=n_{1}^{r}+b^{r}, \quad N_{r}^{\prime}=u_{r}+m_{r} n_{1}^{r} .
$$

- If $z$ does not divide $h$, that is $\varepsilon=0$, we can express $h_{\gamma_{1}}=x^{u_{1}} \tilde{h}_{1}$ where $\tilde{h}_{1}$ is a product of $m_{1}$ factors of the form $\left(z^{n_{1}^{1}}-\tilde{v} x^{b^{1}}\right)$, again $\operatorname{gcd}\left(n_{1}^{1}, b^{1}\right)=1$. In this case

$$
N_{1}=\left(u_{1}+m_{1} b^{1}\right) n_{1}^{1}, \quad \nu_{1}=\nu n_{1}^{1}+b^{1}, \quad N_{1}^{\prime \prime}=u_{1}+m_{1} b^{1} .
$$

Recall that the inverse of the zeta function of the monodromy is, up to a factor $t$ and $(1-t)$ a polynomial. In order to prove the theorem we must take special care of numerators in the formula (7.1). They can arise in several situations:

(1) $r>1, x$ does not divide $h, v(r)=1$. The equations of (7.2) imply that $\exp \left(-2 i \pi \frac{\nu_{r}}{N_{r}}\right)$ is a root of the polynomial which is the inverse of the quotient $\frac{1-t^{N_{r}^{\prime}}}{1-t^{N_{r}}}$.

(2) $r>1, z$ does not divide $h, v(1)=1$. We proceed using equations (7.3).

(3) $r=1$, either $x$ or $z$ divide $h, v(1)=1$. We proceed using equations (7.2) or (7.3)

(4) $r=1$, neither $x$ nor $z$ divide $h, v(1)=1$. Using equations (7.2) it is easily seen that $\left(N_{1}, \nu_{1}\right)$ provides a root of the inverse of the monodromy zeta function. In such a case the zeta function is written as

$$
\frac{\left(1-t^{N_{1}^{\prime}}\right)\left(1-t^{N_{1}^{\prime \prime}}\right)}{\left(1-t^{N_{1}}\right)} \zeta\left(h_{1,1}\right)(t)
$$

With this arguments, if $\operatorname{depth}(h)=1$, the proof of the result is finished. Let us assume now that $\operatorname{depth}(h)>1$. From the above computations we must prove that $\operatorname{SCP}\left(h_{q, j}\right)$ produce also roots of $(\zeta(h)(t))^{-1}$. From the induction hypothesis this is true whenever $\left(\zeta\left(h_{q, j}\right)(t)\right)^{-1}$ divides $(\zeta(h)(t))^{-1}$. The unique case where this fact does not happen is in (7.4), since the first factor is not a polynomial. Let us rewrite the above formula using (7.2), (17.3) and $u_{1}=u_{r}=0$ :

$$
\frac{\left(1-t^{m_{1} n_{1}^{1}}\right)\left(1-t^{m_{1} b^{1}}\right)}{\left(1-t^{m_{1} n_{1}^{1} b^{1}}\right)} \zeta\left(h_{1,1}\right)(t) .
$$

Since the inverse zeta of the monodromy is, up to a factor $t(1-t)$, a polynomial, it must exist a factor $\left(1-t^{m_{1}}\right)$ which divides $\left(\zeta\left(h_{1,1}\right)(t)\right)^{-1}$. Let us note that $h_{1,1}=$ $x^{m_{1} n_{1}^{1} b^{1}}\left(z^{m_{1}}+\ldots\right)$ and the new differential form is $x^{n_{1}^{1}+b^{1}-1} d x \wedge d z$. The highest vertex of the new Newton polygon is $\left(m_{1} n_{1}^{1} b^{1}, m_{1}\right)$; let us assume that we have $s$ edges and denote $(\tilde{N}, \tilde{\nu})$ the strong candidate pole associated to highest edge. Let us denote $(\tilde{n}, \tilde{b})$ the coprime integers which provide the slope of the edge. 
We have $\tilde{N}=m_{1}\left(\tilde{n} n_{1}^{1} b^{1}+\tilde{b}\right), \tilde{\nu}=\nu_{1} \tilde{n}+\tilde{b}=\left(n_{1}^{1}+b^{1}\right) \tilde{n}+\tilde{b}$. The function $\zeta\left(h_{1,1}\right)(t)$ is

$$
\zeta\left(h_{1,1}\right)(t)=\left(1-t^{\tilde{N}}\right)^{-v(s)} \zeta\left(\bar{h}_{s, j}\right)(t) \prod_{q=1}^{s-1} \Delta_{q},
$$

where $\Delta_{q}$ is the product of the factors associated to the $s-1$ first edges. The factor $\left(1-t^{\tilde{N}}\right)$ is a multiple of $\left(1-t^{m_{1}}\right)$. This means that $(\tilde{N}, \tilde{\nu})$ is the only candidate pole of which may not give an eigenvalue of the monodromy of $h$. Since

$$
(\tilde{N}, \tilde{\nu})=\left(m_{1}\left(\tilde{n} n_{1}^{1} b^{1}+\tilde{b}\right),\left(n_{1}^{1}+b^{1}\right) \tilde{n}+\tilde{b}\right),
$$

it follows that $\exp \left(-2 i \pi \frac{\tilde{\nu}}{\tilde{N}}\right)^{m_{1}}=\exp \left(-2 i \pi \frac{\left(n_{1}^{1}+b^{1}\right) \tilde{n}+\tilde{b}}{\tilde{n} n_{1}^{1} b^{1}+\tilde{b}}\right)$. It easy to check that the rational number $\frac{\left(n_{1}^{1}+b^{1}\right) \tilde{n}+\tilde{b}}{\tilde{n} n_{1}^{1} b^{1}+\tilde{b}}$ is not a positive integer. In particular $\exp \left(-2 i \pi \frac{\tilde{\nu}}{\tilde{N}}\right)^{m_{1}} \neq 1$.

\subsection{Monodromy conjecture: general case.}

We will need some facts about the zeta-function of the monodromy of quasiordinary analytic power series. A formula to compute the zeta function of the monodromy has been obtained by L.J. McEwan, A Némethi, and P.D. González Pérez, see [28, 19].

Assume $h$ is a quasi-ordinary power series defined by $f(\mathbf{x}) u(\mathbf{x}, z)$ with $u(0) \neq 0$ and $f=z^{n}+a_{1}(\mathbf{x}) z^{n-1}+\ldots$ is in good coordinates. If $\Lambda_{C E}=\emptyset$ then $f$ is irreducible and smooth. Otherwise one reorders the variables $\mathbf{x}$ in such a way that the first entry of $\min \Lambda_{C E} \in \mathbb{Q}^{d}$ is non-zero. Since the set $\Lambda_{N D}(f) \subset \Lambda$ is totally ordered, $\min \Lambda_{C E}$ exists.

Theorem 7.2. 28] [19] With the above hypothesis, after the reordering of the coordinates described before one has $\zeta(h)(t)=\zeta(f)(t)=\zeta\left(\left.f\right|_{\left\{x_{2}=\ldots=x_{d}=0\right\}}\right)(t)$.

In fact, if min $\Lambda_{C E}$ has at least two non-zero entries, then $\zeta(f)(t)=\left(1-t^{n}\right)$.

Corollary 7.3. If $h \in \mathbb{C}\{\mathbf{x}\}[z]$ is quasi-ordinary satisfying $\mathbf{e v}(h)=1$ and $\omega a$ regular differential form such that $(h, \omega)$ satisfies the support condition (2.3), then $Z_{D L}(h, \omega, T)$ verifies the monodromy conjecture.

We can apply Theorem 7.2 and Corollary 6.1 to conclude the proof of the corollary from Theorem [7.1]

Example 7.4. If $f=z^{n}+x_{1} \cdots x_{r}$. Then $f$ has a non-degenerated Newton polyhedron. One can compute the topological zeta function (using Denef and Loeser formula or our algorithm) and the monodromy zeta function $\zeta_{f}(t)$ (using the above result or Varchenko formula, 32 $)$. In fact $\zeta(f)=\left(1-t^{n}\right)$ and $Z_{t o p, 0}(f, s)$ has only two poles $s=-1$ and $s=-\frac{n+1}{n}$. In particular the monodromy conjecture is verified.

Theorem 7.5. Let $h(x, z) \in \mathbb{C}\{\mathbf{x}\}[z]$ be a quasi-ordinary power series and $\omega$ a regular differential form such that $(h, \omega)$ satisfies the support condition [2.3. Then $Z_{D L}(h, \omega, T)$ and $Z_{\mathrm{top}, 0}(h, \omega, s)$ verify the monodromy conjecture. 
Proof. Let $h \in \mathbb{C}\left\{x_{1}, \ldots, x_{d}\right\}[z]$ be a quasi-ordinary analytic power series in good coordinates. We proceed by induction on $\mathbf{e v}(h)=d>1$.

Assume $h \in \mathbb{C}\left\{x_{1}, x_{2}\right\}[z]$ has $\mathbf{e v}(h)=2$. By Proposition 6.10, every strong candidate pole is either $\left(n, n_{1}+1\right)$ or it is a strong candidate pole of one of the transversal sections. In the former case, the highest compact 1-dimensional face of $\Gamma(h)$ is special in both coordinates. Thus min $\Lambda_{C E}(h)$ has at least two nonzero entries, which implies $\zeta(h)(t)=\left(1-t^{n}\right)$, after Theorem [7.2. In particular $\exp \left(-2 i \pi\left(n_{1}+1\right) / n\right)$ is an eigenvalue of the monodromy of $h$ at the origin.

Otherwise, since we have proved the result for $\operatorname{ev}(h)=1, \exp \left(-2 i \pi\left(n_{1}+1\right) / n\right)$ is an eigenvalue of the monodromy of one of the transversal sections at some point which is, in fact, contained in $h^{-1}(0)$.

Moreover for any quasi-ordinary analytic power series $h$ such that $\mathbf{e v}(h)=2$ the monodromy conjectures for motivic and topological zeta functions hold, after Corollary 6.1.

By Proposition 6.11 and the induction we can conclude that the monodromy conjecture for motivic and topological zeta functions hold for quasi-ordinary power series $h$ such that $\mathbf{e v}(h)=d$.

\subsection{Monodromy conjecture for the Igusa zeta-function.}

Let $p$ be a prime number and let $K$ be a $p$-adic field, i.e $\left[K: \mathbb{Q}_{p}\right]<\infty$. Let $R$ be the valuation ring of $K, P$ the maximal ideal of $R$, and $\bar{K}=R / P$ the residue field of $K$. Let $q$ denote the cardinality of $\bar{K}$, so $\bar{K} \simeq \mathbb{F}_{q}$. For $z$ in $K$, let ord $z$ denote the valuation of $z$, and set $|z|=q^{\text {-ord } z}$. Let $h$ be a non constant element in $K\left[x_{1}, \ldots, x_{d}\right]$. The $p$-adic Igusa local zeta function $I_{0}(h, K, s)$ associated with $h$ (relative to the trivial multiplicative character) is defined as the $p$-adic integral

$$
I_{0}(h, K, s):=\int_{P R^{d}}|h(x)|^{s}|d x|,
$$

for $s \in \mathbb{C}, \Re(s)>0$, where $|d x|$ denotes the Haar measure on $K^{d}$ normalized in such of way that $R^{d}$ is of volume 1 . Igusa proved that $I_{0}(h, K, s)$ is a rational function on $q^{-s}$, see 8 .

Remark 7.6. The method described here can be used to computed $I_{0}(h, K, s)$ in case $h \in K\left[x_{1}, \ldots, x_{d}, z\right]$ be a quasi-ordinary polynomial.

Assume now that $h$ is a non-constant polynomial in $F\left[x_{1}, \ldots, x_{d}\right]$, for some number field $F \subset \mathbb{C}$. Igusa's monodromy conjecture states that for almost all $p$-adic completion $K$ of $F$, if $s_{0}$ is a pole of $I_{0}(h, K, s)$, then $\exp \left(2 i \pi \Re\left(s_{0}\right)\right)$ is an eigenvalue of the local monodromy of $h$ at some point of $h^{-1}(0)$.

It is known and deduced from [13, see also [15], that for almost all finite places of the number field $F$, the real parts $N s+\nu$ of poles of $I_{0}(h, K, s)$ come from factors $\left(1-\mathbb{L}^{-\nu} T^{N}\right)$ in the denominator of $Z_{\text {naive }}(h, T)$ (which it is essentially nothing but $\left.Z_{\mathrm{DL}}(h, T)\right)$. In particular our proof can be applied to polynomials $h \in F[\mathbf{x}, z]$ with coefficients in a number field $F$ which are quasi-ordinary polynomials. Then the Igusa monodromy conjecture is also true in this case. 


\section{REFERENCES}

[1] S.S. Abhyankar, On the ramification of algebraic functions, Amer. J. Math. 77 (1955), 575592.

[2] E. Artal Bartolo, Pi. Cassou-Noguès, I. Luengo, A. Melle Hernández, Monodromy conjecture for some surface singularities, Ann. Scient. Éc. Norm. Sup. 35 (2002), 605-640.

[3] A. Barvinok, J.E. Pommersheim, An algorithmic theory of lattice points in polyhedra, New perspectives in algebraic combinatorics, Berkeley, CA, 1996-97), 91-147, Math. Sci. Res. Inst. Publ., 38, Cambridge Univ. Press, Cambridge (1999), 91-147.

[4] D. Bernstein, A.G. Khovanskii and A.G. Kushnirenko, Newton polyhedra, Usp. Met. Nauk. 31 (1976), 201-202.

[5] Pi. Cassou-Noguès, A. Płoski, Algebraic Curves, Book in preparation.

[6] J. Cheah, On the cohomology of Hilbert schemes of points, J. Algebraic Geometry 5 (1996), 479-511.

[7] J. Denef, The rationality of the Poincaré series associated to the p-adic points on a variety, Invent. Math. 77 (1984), 1-23.

[8] _ Report on Igusa's local zeta function, Séminaire Bourbaki, Astérisque 201-202-203 (1991), 358-386.

[9] _ Poles of p-adic complex powers and Newton polyhedra, Nieuw Archief voor Wiskunde 13 (1995), 289-295.

[10] J. Denef, K. Hoornaert, Newton polyhedra and Igusa's local zeta function, J. Number Theory 89 (2001), no. 1, 31-64.

[11] J. Denef, F. Loeser, Weights of exponential sums, intersection cohomology, and Newton polyhedra, Invent. Math. 106 (1991), 275-294.

[12] Caractéristiques d'Euler-Poincaré, fonctions zêta locales et modifications analytiques, J. Amer. Math. Soc. 5 (1992), no. 4, 705-720.

[13] _ Motivic Igusa Zeta functions, J. Algebraic Geometry 7 (1998), 505-537.

[14] , Germs of arcs on singular algebraic varieties and motivic integration, Invent. Math. 135 (1999), 201-232.

[15] _ Geometry on arc spaces of algebraic varieties, Proceedings of 3rd European Congress of Mathematics, Barcelona, Progress in Mathematics 201 (2001), Birkhäuser, 327-348.

[16] _ Motivic integration, quotient singularities and the McKay correspondence, Compositio Math. 131 (2002), 267-290.

[17] Y.-N. Gau, Embedded topological classification of quasi-ordinary singularities, Mem. Amer. Math. Soc. 74 (1988), no. 388, 109-127.

[18] P.D. González Pérez, Étude des singularités quasi-ordinaires d'hypersurfaces au moyen de la géométrie torique, Thèse de Doctorat, Paris 7, 2002.

[19] P.D. González Pérez, L.J. McEwan, A. Némethi, The zeta function of a quasi-ordinary singularity II, preprint.

[20] G. Guibert, Espaces d'arcs et invariants d'Alexander, Comment. Math. Helv. 77 (2002), $783-$ 820.

[21] K. Hoornaert, D. Loots, A computer program to calculate Igusa's Local Zeta Function and the Topological Zeta Function for non-degenerated polynomials, freely available at http://www.wis.kuleuven.ac.be/algebra/kathleen/program.htm.

[22] J. Igusa, Complex powers and asymptotic expansions I, J. reine angew. Math. 268/269 (1974), 110-130; II, ibib. 278/279 (1975), 307-321.

[23] S. Ishii, J. Kollár, The Nash problem on arc families of singularities, (2002), preprint available at arXiv:math.AG/0207171.

[24] J. Lipman, Topological invariants of quasi-ordinary singularities, Mem. Amer. Math. Soc. 74 (1988), no. 388, 1-107. 
[25] F. Loeser, Fonctions d'Igusa p-adiques et polynômes de Bernstein, Amer. J. Math. 110 (1988), no. $1,1-21$.

[26] _ Fonctions d'Igusa p-adiques, polynômes de Bernstein, et polyèdres de Newton, J. reine angew. Math. 412 (1990), 75-96.

[27] I. Luengo, A new proof of the Jung-Abhyankar theorem, J. Algebra 85 (1983), 399-409.

[28] L.J. McEwan, A. Némethi, The zeta function of a quasi-ordinary singularity I, to appear in Compositio Math.

[29] B. Rodrigues, W. Veys, Holomorphy of Igusa's and topological zeta functions for homogeneous polynomials Pacific J. Math. 201 (2001), no. 2, 429-440.

[30] J. Sebag, Rationalité des séries de Poincaré et des fonctions zêta motiviques, (2002), preprint available at arXiv:math.AG/0212025.

[31] R. Stanley, Enumerative combinatorics. Vol. I With a foreword by Gian-Carlo Rota. The Wadsworth \& Brooks/Cole Mathematics Series. Wadsworth \& Brooks/Cole Advanced Books \& Software, Monterey, CA, 1986.

[32] A Varchenko, Zeta function of monodromy and Newton's diagram, Invent. Math. 37 (1976), 253-262.

[33] W. Veys, Determination of the poles of the topological zeta function for curves, Manuscripta Math. 87 (1995), 435-448.

[34] _ Zeta functions and "Kontsevich invariants" on singular varieties, Canad. J. Math. 53 (2001), no. 4, 834-865.

[35] O. Villamayor U., On equiresolution and a question of Zariski, Acta Math. 185 (2000), no. 1, $123-159$.

Departamento de Matemáticas, Universidad de Zaragoza, Campus Pza. San FranCISCO S/N, E-50009 ZARAGOZA SPAIN

E-mail address: artal@unizar.es

Institut de Mathématiques de Bordeaux, Université Bordeaux I, 350, Cours de la Libération 33405 Talence Cedex, France.

E-mail address: cassou@math.u-bordeaux.fr

Departamento de Álgebra, Universidad Complutense, Plaza de Ciencias 3, E28040 MADRID SPAIN

E-mail address: iluengo@mat.ucm.es, amelle@mat.ucm.es 